SF

103

M99

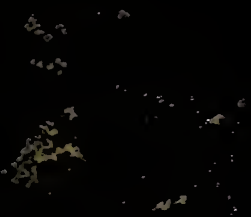




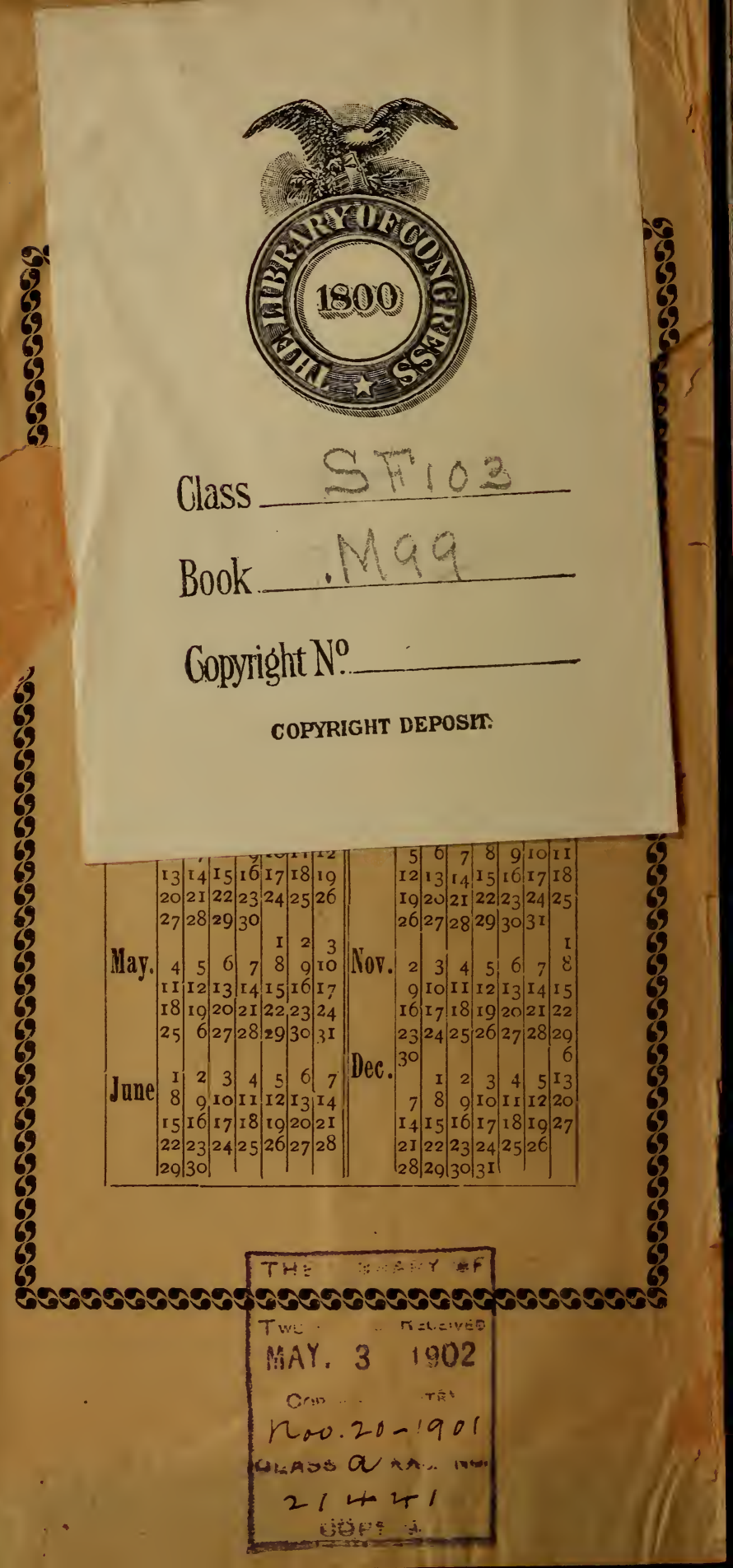




\section{The \\ Stockman's \\ Brand Book}

KEYA PAHA COUNTY

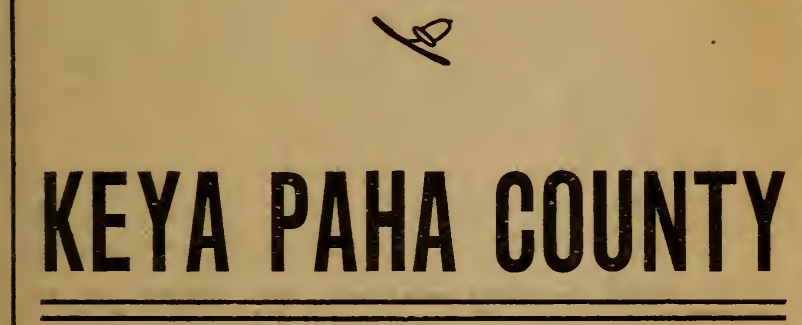

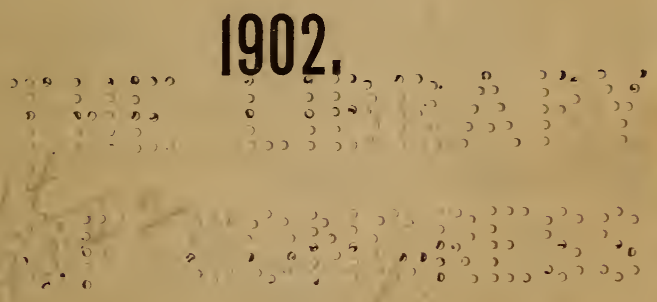

By OTTO MUTz.

Copyright, 1901, by Otto Mutz.

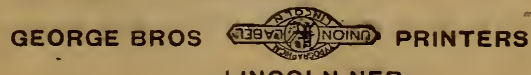
LINCOLN NEB. 


\section{Dreface.}

The Stockman's Brand Book is dedicated to the Stockmen of the Northwest.

The livestock interests of this section have attracted to it much Capital, and many of the brightest and most energetic men of the country.

Identity of interests has called into existence many organizations for the better protection of owners against loss by theft and straying. In response to their demands the State Legislature passed a State Brand Law in 1899 the object of which is to prevent conflictions in brands between the distinctly stóck-raising counties, but at this time only a small per cent of the stock owners have.procured a brand under the state law. Many are using brands granted under the old county law, while many have never been recorded anywhere.

Under the state law, there is no means of knowing the brands of your county without consulting the records at the State Capitol.

The estray laws of the state are slow in returning to owners of estrays evidence of their whereabouts: These facts are responsible for calling into exîstence this little brand book.

Withothe Brand Book in youbr: poadiet, you

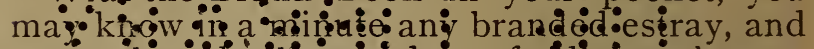
a postal cardevill saré days of priding and many times a valuable animal.

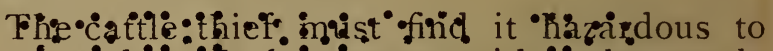

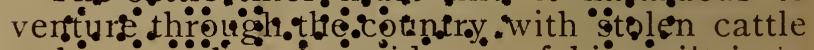
or horses, when the evidence of his guilt is in every man's pocket.

I make no apology for imperfections.

I have earnestly endeavored to get every brand in the county, and to get it recorded accurately.

The work has been done under many difficulties. When the book has shown its value as a Stock-hunter, it is hoped that a more complete edition will be possible.

The Author. 


\section{Some Nebraska Laws Relating to Animals.}

The following laws relating to stock are selected and prepared in a condensed form with the greatest care. I have endeavored to insert those most needed by men engaged in cattle raising.

\section{Marks and Brands}

Chap. 51. Sec. I, Compiled Statutes 190I provides: "Any person having cattle, hogs, sheep, horses, mules or asses shall have the right to adopt a brand or mark for the use of which he shall have the exclusive right in this state, after recording such brand or mark with the Secretary of State.

Sec. The fee for recording such brand is one dollar and fifty cents.

Sec. 4. No person shall have or adopt a brand or mark previously recorded under this act to another person if used on the same side. Neither shall the Secretary of State record the same brand or mark to more than one person, unless for opposite sides.

Sec. 5. Application for State brand. See County Clerk.

Sec. 6. Conflicting brands. The authority of deciding all questions of confliction rests with the Secretary of State.

"No brand described as being on either side of the animal shall be accepted."

"A brand described as being on both sides may be accepted."

Sec. 7. Provides for examination of evidence of confliction by Secretary of State, and notification of parties using conflicting brands.

Sec. 8. Provides: That parties bringing into any county, stock for grazing purposes, shall 
brand them with a brand that shall be a full and distinguishing mark from all other brands then recorded. Failure to comply with this section lays party liable for all resulting damages.

Sec. 9. Brands rejected. All brands shall be rejected that in addition to a brand recorded has any of the following: a straight bar, a quarter, half, or entire circle, or diamond, either upright or inverted, whether placed across, above, below, at either side, or encircling the main brand.

All repetitions of letters, numbers and figures on the same side of the animal previously recorded shall be rejected.

All brands known as solid brands shall be rejected. Combinations of letters, numbers or figures may be permitted, although the same may have been recorded single or together if in the judgment of the Secretary of State said combination is so different from any prevous record as to constitute a new brand with no danger of infringement.

Sec. Io. In all suits at law or in equity or in any criminal proceedings, the brand shall be prima-facia evidence of ownership. Provided: That such brand shall be duly recorded as provided by law .

Sec: II. Violation of act. Any person or persons who shall violate or fail to obey the provisions of this act, or shall continue the use of a brand or mark after it has been rejected by said Secretary of State, or shall continue the use of any brand or mark after said Secretary of State shall have decided that the same conflicts with a previously recorded brand or mark shall be deemed guilty of a misdemeanor, and upon conviction shall be punished by a fine of not exceeding one thousand dollars, (\$1000.00) or imprisonment in the County Jail for a term not exceeding one year or both in the discretion of the court. 


\section{Estray Law.}

Any person holding land in this State, by deed, title bond or lease for one or more years and being in possesion thereof, may take up any estray found within his enclosed premises at any season of the year.

He may take up any estray found around his premises between the 2oth day of October and the first day of April.

Any person at any time, may take up any horse, mule or ass with any portion of harness attached to them or any oxen with yoke that are believed to have estrayed.

\section{Record of Description.}

Party taking up an estray shall send a description of the same to a Justice of the Peace in the precinct within forty-eight hours and said Justice of the Peace shall record the same in a book kept by him for that purpose.

If estray is not claimed within ten days thereafter party shall file the description with the County Clerk of the County. In twenty days thereafter the description shall be published in a newspaper, published within the county for five consecutive weeks. Publication fee is three dollars. If the number advertised at one time exceeds three, publisher may receive one dollar for each estray above that number.

Owner may reclaim at any time before the sale by proving property and paying costs.

\section{Disposition.}

If a sheep, swine or calf under the age of one year has not been reclaimed within six months after advertising it becomes the property of the taker-up without further proceedings.

If the estray be a horse, mule, ass, bull, cow or steer over the age of two years it must be reclaimed within six months after the time it 
was first advertised. If the estray is an animal over the age of one year and not over the age of two years, it must be reclaimed within six months from the time it was first advertised.

Estrays included in the two named classes not reclaimed within the specified times, takerup shall notify the Justice of the Peace who shall appoint two disinterested appraisers, who shall appraise the stock at its true value under oath and make return of the same to said Justice.

Justice shall then appoint a time and place of sale and publish the same in a newspaper printed in the county for five weeks, and post written or printed notices in three public places in the precinct where the estray is to be sold.

If no newspaper is printed in the county, three additional notices shall be posted at the County Seat.

On the day of sale Justice shall sell to the highest bidder in cash. The proceeds after deducting costs and expenses shall be paid to the County Treasurer within ten days after sale to be placed to the credit of the General School Fund of the County, if not claimed within one year from day of sale. Sale shall take place at the residence of taker-up.

Estray must bring at least two-thirds of appraised value, or be reappraised and again offered one week later without further notice.

If appraisers think the animal will not bring more than enough to pay the expenses of sale and advertisement, sale shall be dispensed with and taker-up shall upon payment of expenses be the owner.

Fee of Appraisers $\$ 50$

Fee of Justice. I 50

Sec. I4. The advertisement, the appraisment, and the service of the Justice of the Peace shall be paid by the person taking up the estray and he shall receive the same with fifty 
per cent additional from the proceeds of the sale of the estray.

Parties taking np estrays are not liable for death unless it was the result of mistreatment or neglect.

\section{Herd Law.}

Chap. 2. Art. 3. Sec. 2. Compiled Statute Igor, Provides: That all damages to property by stock shall be paid by the owner.

Taker-up has a lien upon animals for damagés and costs.

Sec. 3. Provides for giving notice to owner of stock impounded. When owner is known the following notice should be given by leaving at his usual place of residence, with some members of his family over the age of fourteen or by posting a copy on the door of owner's residence.

\section{NOTICE SALE.}

Mr. ....................... You are hereby notified hat on this ..................................... of of which I now have in my possession (here describe animals), did trespass upon my land and damage the same to the amount of $\$$ You are requested to pay the above charges within forty-eight hours from the delivery of this notice or the aforesaid stock will be sold according to law. I have appointed Mr.

to act as arbitrator should you not feel satisfied with the amount of damages claimed in the within notice.

Signed

No claim for damages can be maintained unless notice has been given.

If owner refuses to appoint an arbitrator and does not pay damages within forty-eight hours after having been notified in writing, taker-up should fle with any Justice of the Peace said amount of damages and costs, and the animals shall be sold upon execution as required by law. 
Sec. 2. "In case the parties cannot agree as to the amount of damages and cost sustained, each party may choose a man, and in case the two cannot agree they should choose a third man, who after being duly sworn for the purpose herein named, they shall proceed to assess the damages possessing for that purpose the general powers of arbitrators."

Sec. 6. The award shall be made in writing. If the award is not paid in five days it may be filed with any Justice of the Peace in the county, and shall operate as a judgment, which judgment shall act as a lien upon the stock taken up. Execution may issue upon said stock for the-collection of said damages and costs.

Either party may appeal.

Arbitrators fees are two dollars each.

Tender by owner before trial of an amount equal to award by arbitrators and costs, will not release him from payment of costs.

Sec. 7. In case the owner is not known, taker-up shall proceed as provided by law regarding estrays.

Sec. 8. "That cultivated lands within the meaning of this act; shall include all forest trees, fruit trees and hedge rows planted on said lands, also all lands surrounded by a plowed strip not less than one rod in width, which strip shall be plowed at least once a year.

\section{Hide Registry Law.}

Sec. I3, Chap. 5I, Compiled Statutes, IgOI, page 789 .

Butcher's Record. Every butcher shall keep a record of all animals slaughtered, giving age, sex, marks and brands, of whom purchased. and date of purchase, and said record shall at all times be open for public inspection at his place of business.

Hides Exhibited. It is unlawful for persons who slaughter cattle for beef and offer for sale 
said beef without exhibiting the hide at the time and place said beef is offered, and the brands must not be changed, mutilated or destroyed.

Parties slaughtering beef for home consumption shall keep the hide of the slaughtered animal not less than three days subject to inspection by any person or persons.

Hide Purchaser's Record. All purchasers of hides of cattle shall keep a record of all hides purchased, which shall state the name of the person from whom purchased, residence, date of purchase, and marks and brands on said hides which shall at all times be open to inspection of stock growers.

Violation; Penalty. Wilful violation or neglect to comply with the provisions of this act is a misdemeanor.

Penalty. Imprisonment in the County Jail not exceeding three months or pay a fine not exceeding one hundred dollars.

\section{Criminal code Relating to Animals.}

Altering ear marks or brands. Chap. Io, Sec. 63. Criminal Code Igor.

If any person shall wilfully and maliciously alter or deface any artificial ear mark or brand upon any horse, mare, foal, filly, mule or ass, sheep, goat or swine, cow, ox, steer, bull or heifer, the property of another every person so offending shall be fined in any sum not exceeding fifty dollars, and be liable in triple damages to the party injured.

Sec. 64. If any person shall unlawfully and maliciously kill or destroy an animal of the value of thirty-five dollars or more the property of another, he shall be imprisoned in the penitentiary not more than three years nor less than one year.

Sec. 65. If the value is less than thirty-five dollars, the fine shall be not less than five 
dollars nor more than one hundred dollars, or imprisonment in the county jail not exceeding three months.

The statutes also make it a criminal offense

To poison animals.

To inhumanly beat animals.

To over-drive, torture, torment, over-load or over-work.

To starve.

To impound for any purpose and neglect to feed or water.

To abandom sick animals to die.

To run horse races in the public road.

To sell or let diseased animals run at largc.

To allow diseased animals to come in contact with others.

To disturb or set at liberty animals impounded in a lawful manner.

\section{Prairie Fires.}

Sec. 62. Criminal code makes it a misdemeanor to wilfully and intentionally or negligently and carelessly set on fire any woods, prairies or other grounds in any part of $\mathrm{Ne}$ braska. The fine for such an offense is not less than five dollars nor more than one hundred dollars and by imprisonment in the county jail not less than one month and not more than six months.

Party may set fire between the first day of March and the last day of November, on land adjoining his own farm for necessary preservation there of from accident by giving his neighbors two days notice of such intention.

Party is liable in a civil action for any damages done in consequence. 


\section{Nebraska Statute of Weights and Measures.}

Barley...................... 48 lbs.

Beans (white) ................ 60

Bran ..................... 20

$\begin{array}{lllll}\text { Corn }(\text { ear }) \ldots \ldots \ldots \ldots & \ldots & \ldots & \ldots & \ldots\end{array}$

Corn (shelled) ................ . 56

Ha凡 (per ton)................. . 2000 "

Oats................... 32

Onions...................

Potatoes (irish) ............... 60

Potatoes (sweet)................ 50

Rre..................... . 56

Seed (blue grass) ......... .... 14

Clover...................... 60

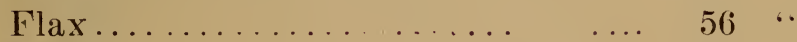

Millet..................

Sorghum.................

Turnips................... . 55 “

Wheat ....................

Alfalfa Seed ................. 


\section{COUNTING TABLE.}

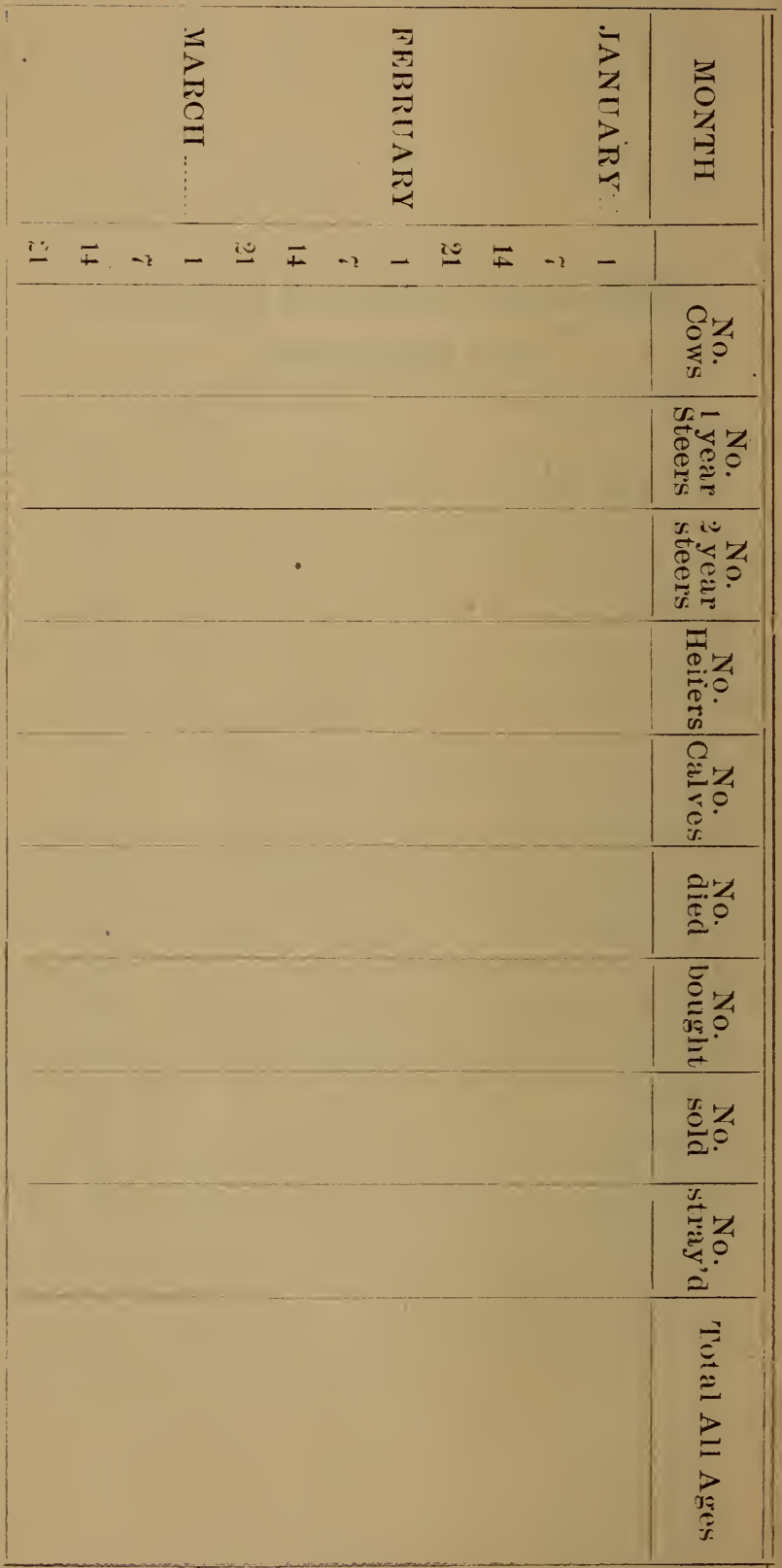




\section{COUNTING TABLE.}

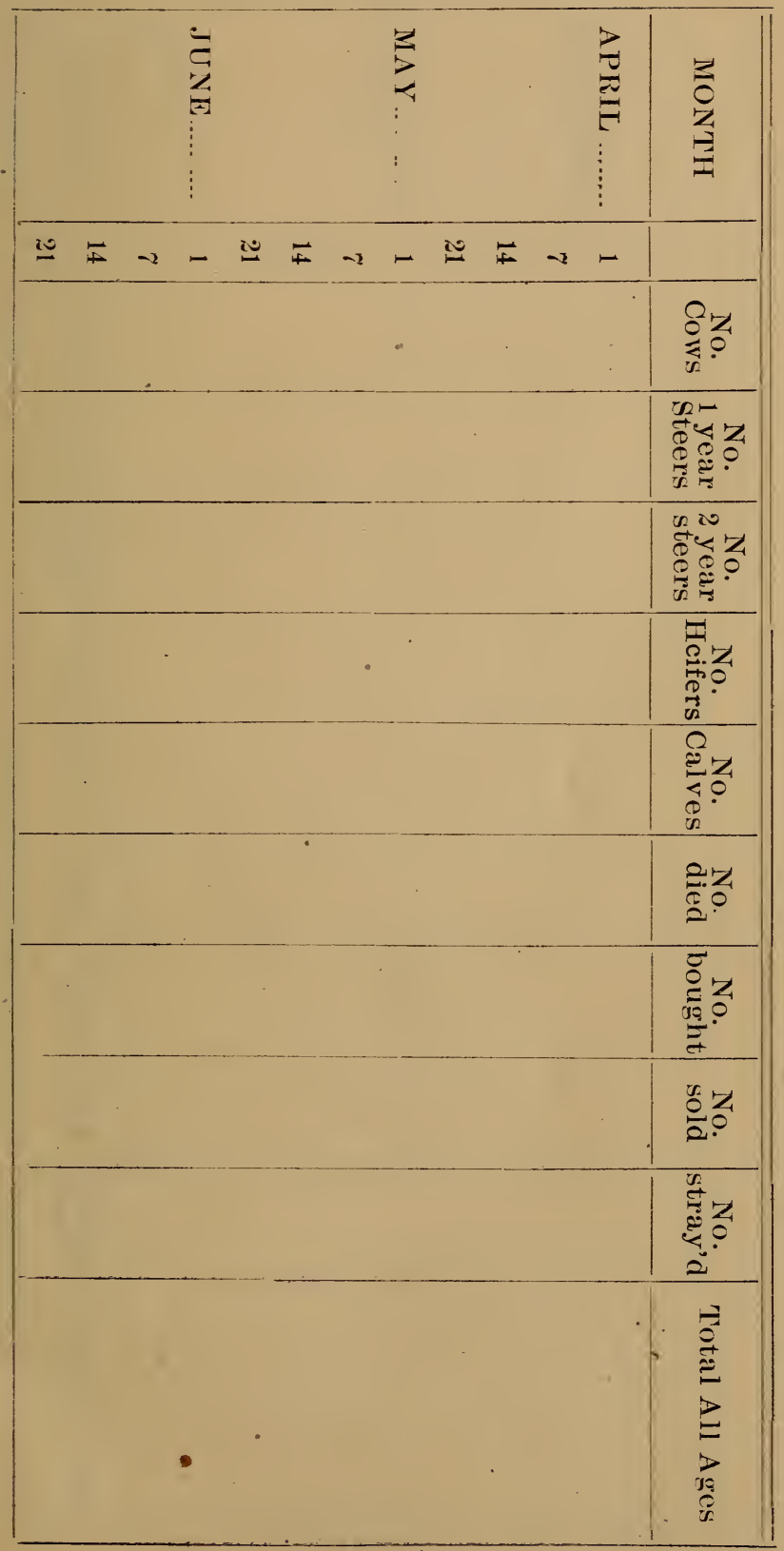


COUNTING TABLE.

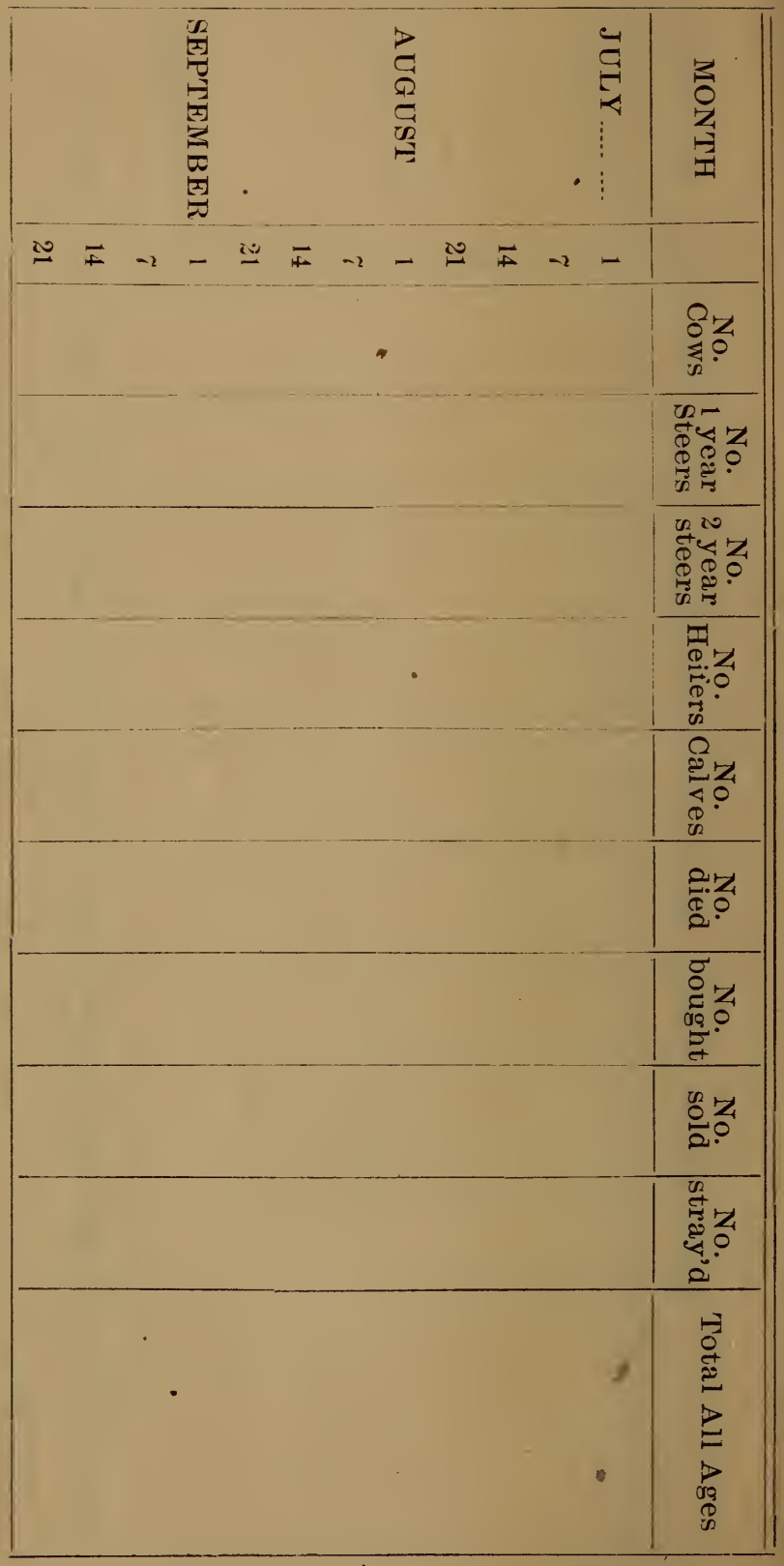




\section{COUNTING: TABLE.}

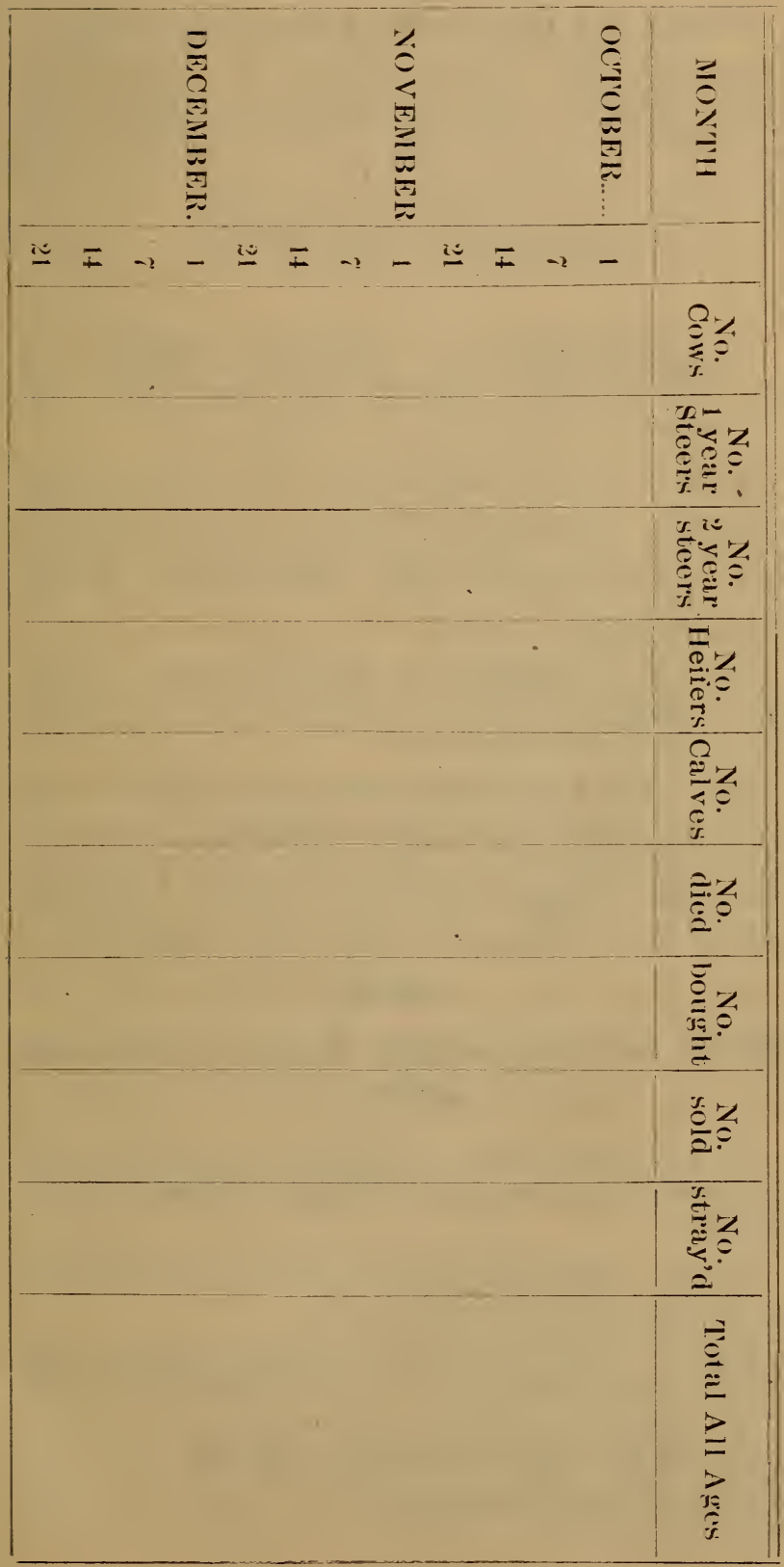




\section{Rules for measuring Hay in Stack.}

We give below some rules for measuring hay in the stack. The rules are in common use but the conditions of hay are so different and the shape of stacks so varied that no rule can be relied upon as perfectly accurate.

In well settled hay $343 \mathrm{cu}$. feet will make a ton.

Prairie hay in stack is generaly bought and sold when settled $425 \mathrm{cu}$. feet per ton. Unsettled prairie hay $512 \mathrm{cu}$. feet per ton.

Rule I. Divide the sum of the distance over the stack and the width by 4 . Multiply this result by itself and that product by the length. and divide by 343 or 425 . The result is in tons and $\mathrm{cu}$. feet.

Rule II. Multiply the product of the length by the breadth by $\frac{1}{3}$ the distance over the stack and divide by 343 or 425 .

Rule III. To measure round stacks. Divide the distance around the stack by 4 and the distance over by 3 . This gives the square at base and height which multiplied together gives the cu. feet. Divide by 343 or 425 .

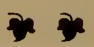

\section{Rules for measuring Ear Corn in the erib.}

To get the number of cu. feet. Multiply the length by the breadth and that product by the height.

Rule I. Multiply the cu. ft. by 4 and divide by 9 , the resnlt is in bushels.

Rule II. Multiply the cu. ft. by $4 \frac{1}{2}$ and cut off the right hand figure, the result is in bus.

To measure wheat, shelled corn or oats in a wagon box or bin. Multiply the cu. ft. by 8 and for greater accuracy add $\frac{i}{2}$ bu. for every $100 \mathrm{cu}$. ft. 


\section{State Farmers' Mutual}

\section{Insurance Company.}

\section{South Omaha, - Nebraska.}

A purely Farmers Mutual Company. Insures only farm property against Fire, Lightning and Tornadoes, for one per cent, and $\mathbf{\$ 2 . 0 0}$ Membership Fee. (Perpetual Policy) subject to Assessment. Has over $\$ 13,500,000$ insurance in force. Live Stock Insur= ance, a special feature. We pay in case of loss, just what you are in= sured for. If you insure your herd for one half $\cdot$ its value, we pay one= half the loss. If three=fourths of its value, we pay three-fourths of the loss.

We want agents where not repre= sented.

Reference, the Banks of this eity.

T. B. HOLMAN, Pres.

B. R. STOUFFER, Sec'y, South Omaha, Neb. 


\section{Inutuly 'q7amen' of}

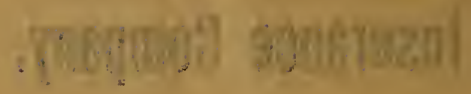

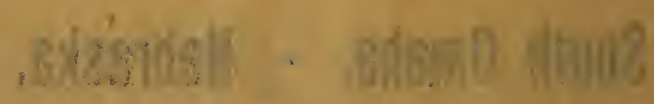

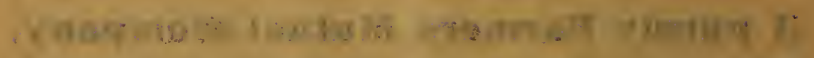

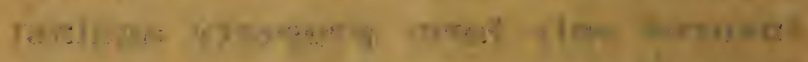

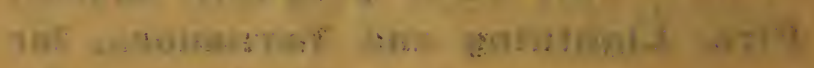

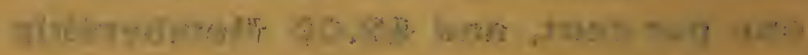

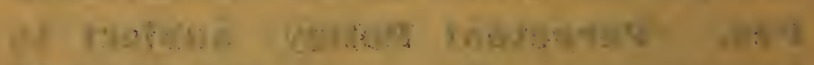

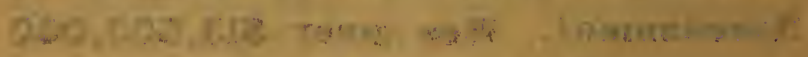

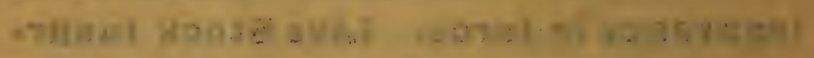

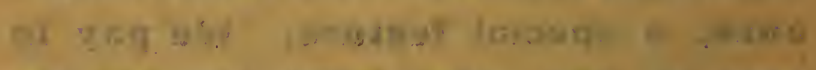

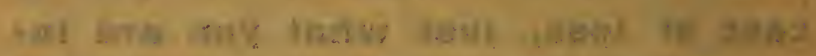

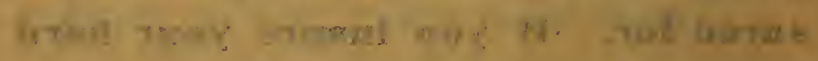

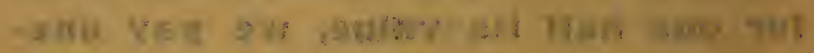

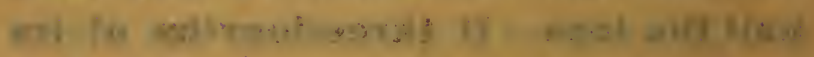

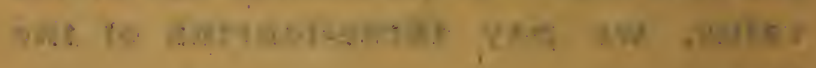

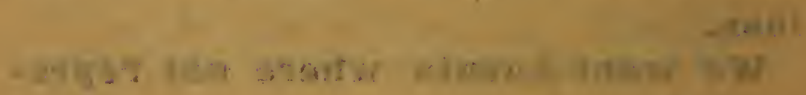

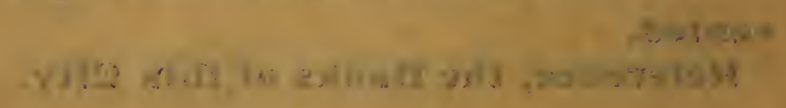

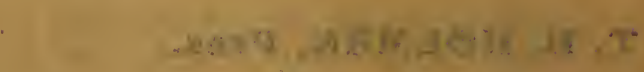

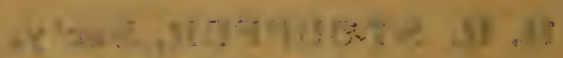
was conanedy ation. 


\section{Burton Precinet.}

Delonging to members of the Keya Paha River Stock Growers' As= sociation are branded as in W. C. Brown's record on this page. The brand is read $\mathbf{7} \mathbf{U} \mathbf{S}$. Members of the Association are designated in the book by the word "Association."

“'The words 'Durham,' 'Hereford' and 'Mixed' as used in the following pages are to designate the breed of stock raised by each individual."

\section{Bammerlin, Adam}

Springview.

Range, Dutch Flat

Read, $\mathrm{X} \quad$ Size $4 \times 4$

Individual.

Durham.
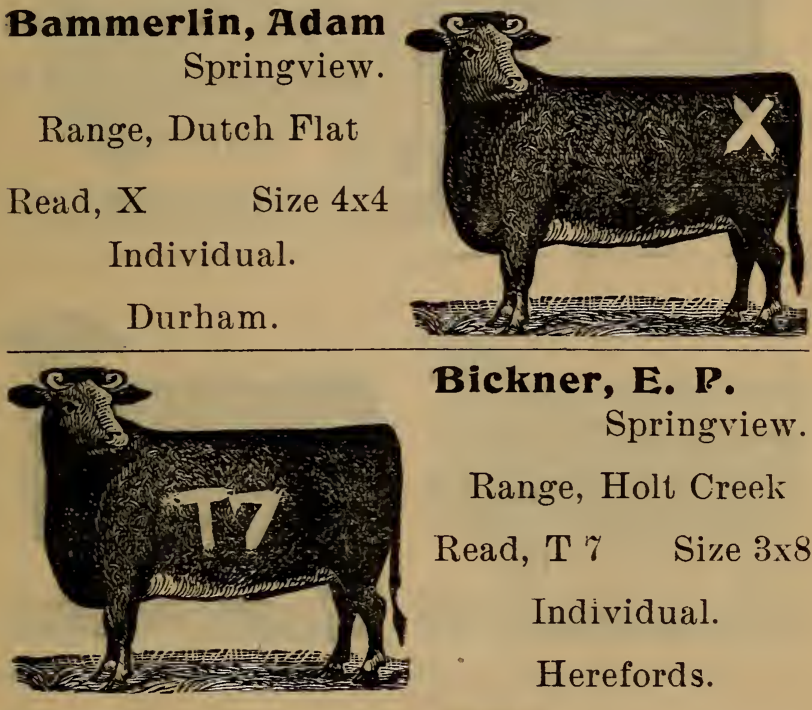

Bickner, E. P.

Springview.

Range, Holt Creek

Read, $T$ $7 \quad$ Size $3 \times 8$

Individual.

Herefords.

\section{Brown, w. e.}

Springview.

Range, Burton Creek

Read, Hammer $3 \times 4$

Association 7US.

Hereford.

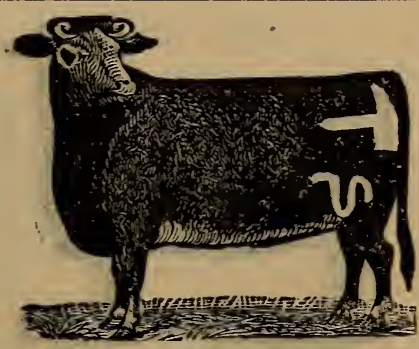


Butler, Mrs. e. Mills.

Range, Keya Paha River

Read, Ace of Heart Individual.

Durham.

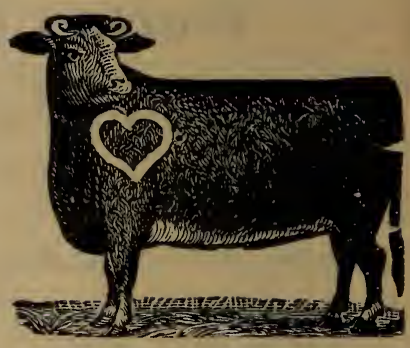

Conner, S. M.

Mills.

Range, Buffialo Creek

Read,J bar T Size $4 \times 12$

Individual.

Hereford.

Demmer, J. H.

Lutes.

Range, Burton Creek

Read, J H D Size 4x6

Individual.

Hereford.

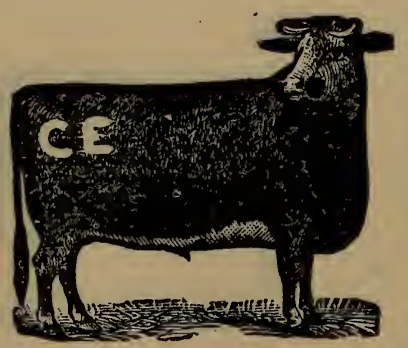

Eweing, e. F.

Springview.

Range, East Holt

Creek

Rẹad, C E Size $4 \frac{1}{2} \times 5$

Individual.

Mixed. 


\section{Horsell, F.}

Springview.

Range, Deer Creek Read, Jug Size 6x6 Individual.

Durham.
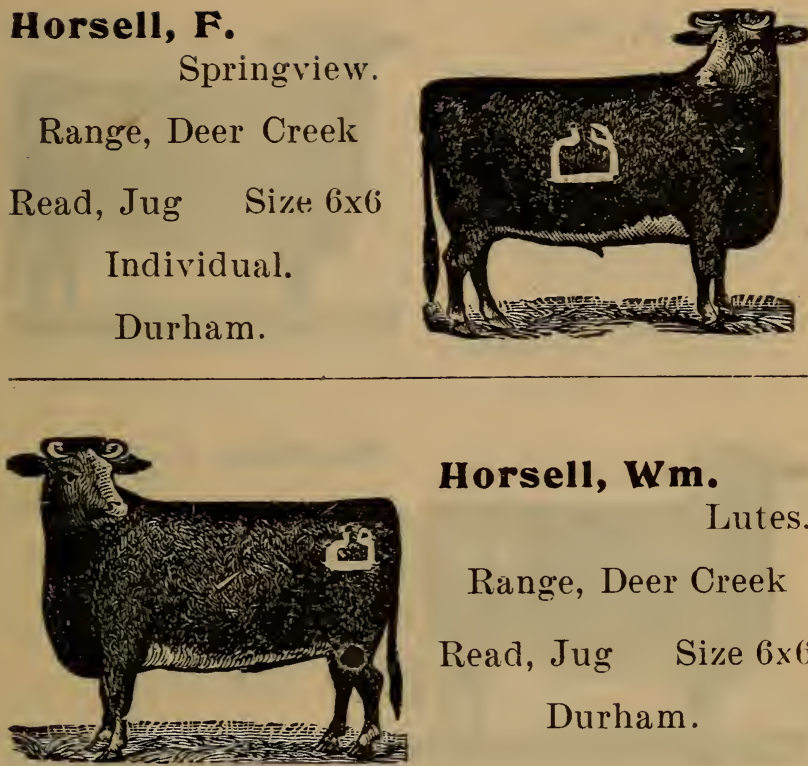

Horsell, Wm.

Lutes.

Range, Deer Creek

Read, Jug Size $6 \times 6$

Durham.

Hills, e. H.

Range, Lutes Creek

Read, J H Size 5x5

Individual.

Hereford.

Lutes.

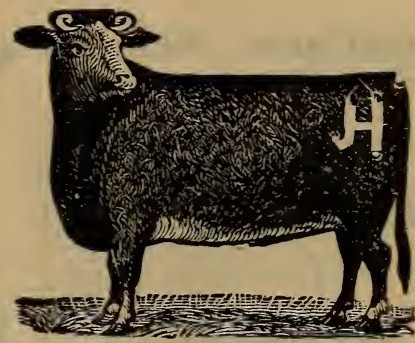

Heiden, $H$.

Lutes.

Read, U 4 Size 6x? State.

Hereford. 
Hiner, K. M.

Range, between Lutes and Mills

Read, H K Size 4x5

Individual.

Durham.
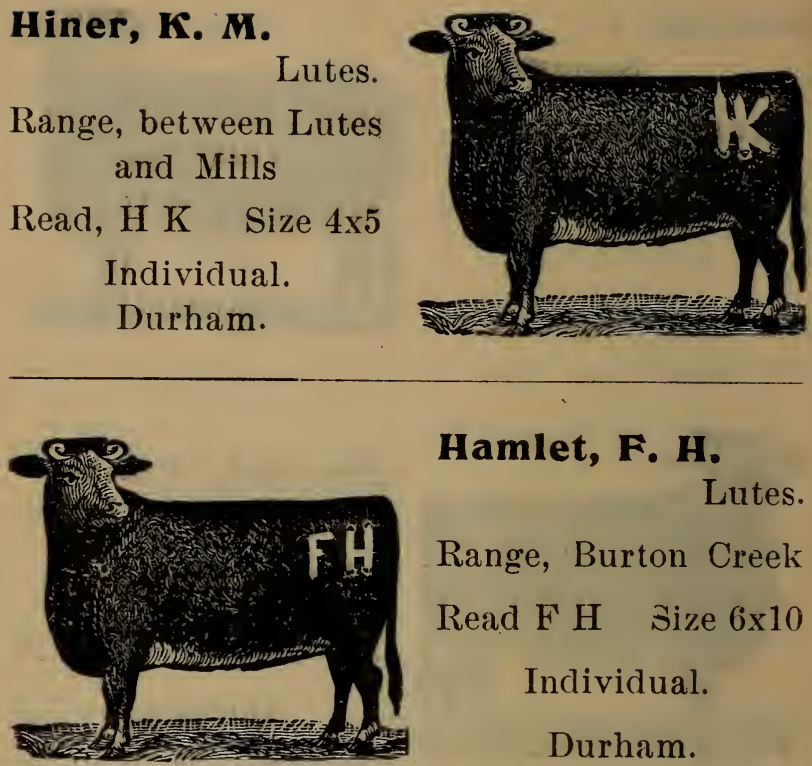

Hamlet, F. H.

Lutes.

Range, Burton Creek Read F H Șize $6 \times 10$ Individual.

Durham.

\section{Hoffman, Mrs. B.} Enterprise.

Range, Dutch Flat . Read, C H Size 5x6

Individual.

Durham.

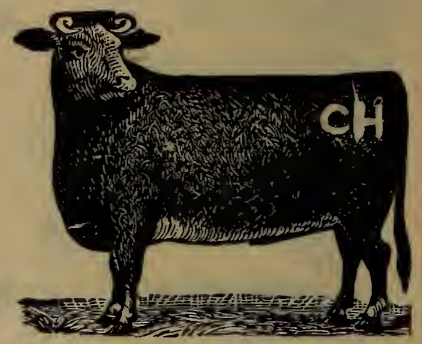

Heiden, Emil

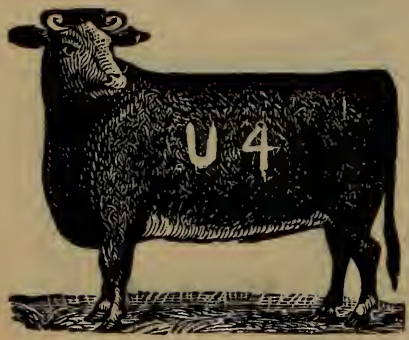

Mills.
Range, Wolf Creek
Read U $4 \quad$ Size $6 \times 9$
State.
Hereford.


Josiassen, e.

Springview.

Range, Burton Creek

Read, C J Size $4 \times 8$

Individual.

Durham.
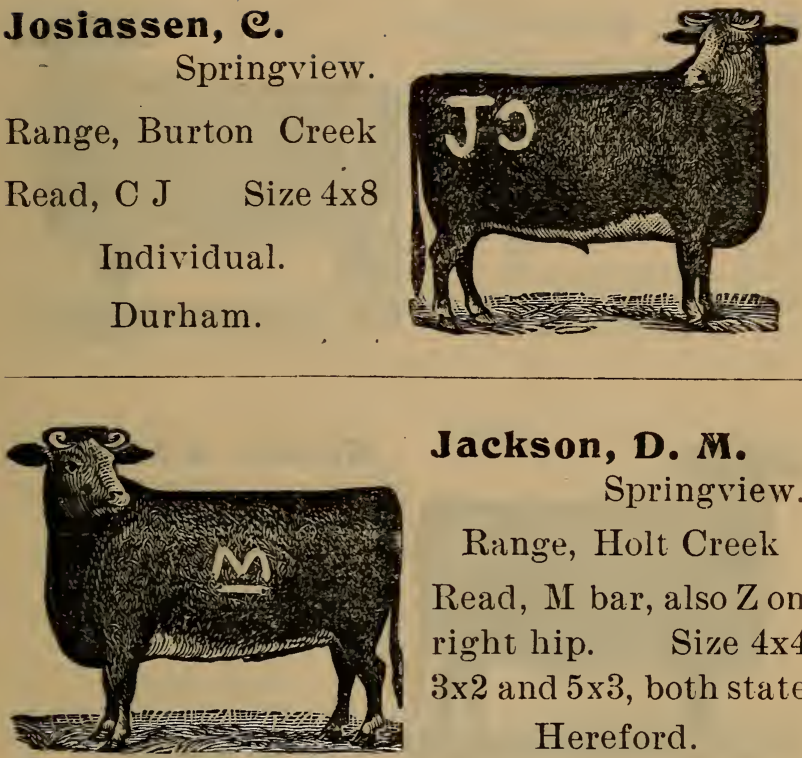

Jackson, D. M.

Springriew.

Range, Holt Creek Read, M bar, also Z on right hip. Size $4 \times 4$ $3 \times 2$ and $5 \times 3$, both state Hereford.

\section{Jackson, J. A.}

Springview.

Range, Burton Creek Read, Z Size 5x3 State. Hereford.

$\mathrm{Z}$ on horses on left shoulder.
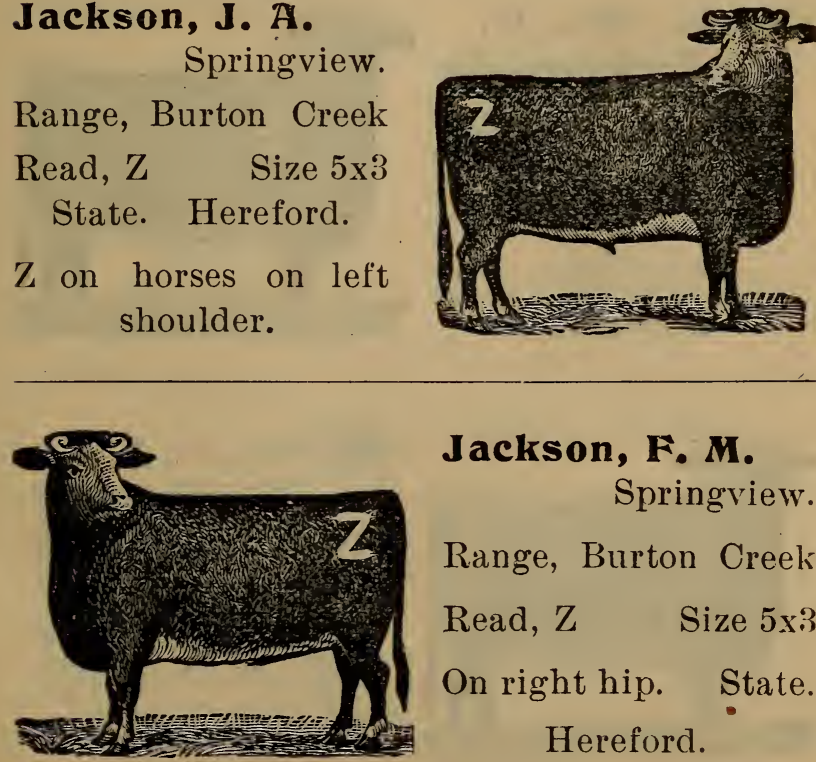

Jackson, F. M.

Springview.

Range, Burton Creek

Read, Z Size 5x3

On right hip. State. Hereford. 
Íirsch, Carl

Enterprise.

Range, Dutch Flat Read, Ship Wheel

State.

Size 5x5

Durham.
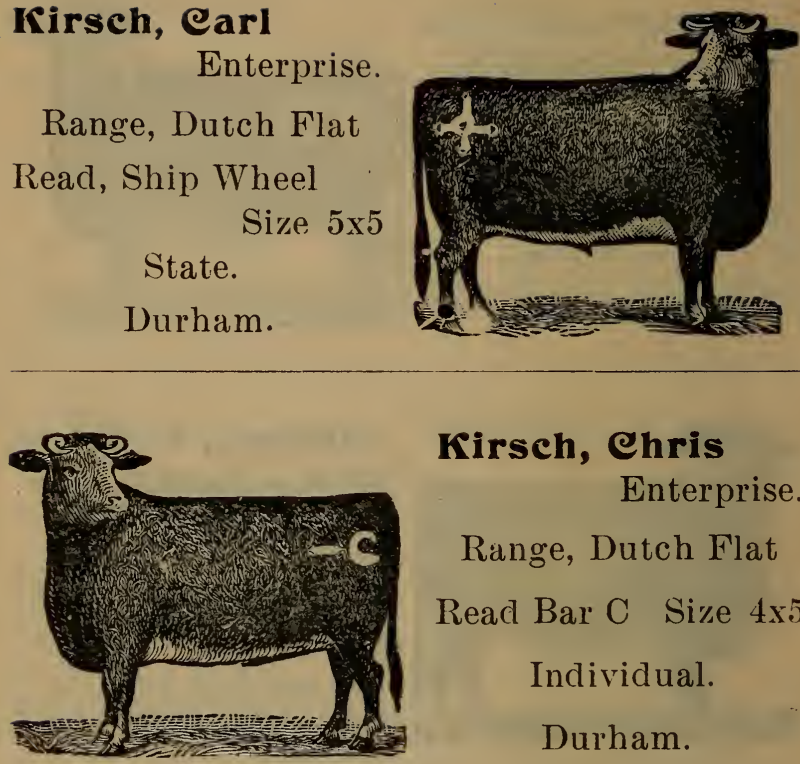

Kirsch, Chris

Enterprise.

Range, Dutch Flat

Read Bar C Size 4x5

Individual.

Durham.

Keinke, Mrs. H.

Enterprise.

Range, Dutch Flat

Read, J K Bar

Individual.

Durham.

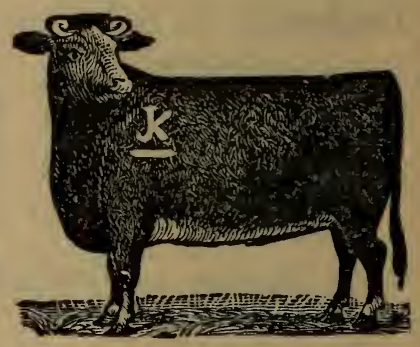

Keinke, e. J.

- Enterprise.

Range, Dutch Flat

Read, One Size 6 in.

Individual.

Durham. 
Leavens, Harry

Lutes.

Range, Lutes Creek Read, $\%$ U K Size 5x State.

Mixed.

Association brand on horses on left Hank.
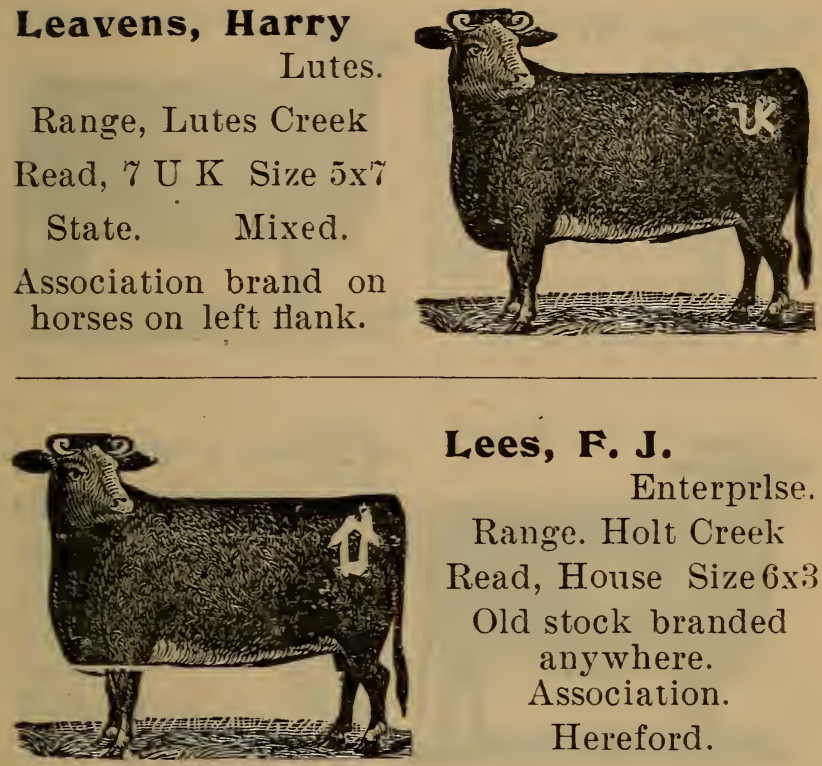

Lees, F. J.

Enterprlse.

Range. Holt Creek

Read, House Size 6x3

Old stock branded anywhere.

Association.

Hereford.

\section{Lewis, Ralph}

Lutes.

Range, Keya Paha River.

Read, Clover Leaf Bar size $6 \times 5$. W. N. Ass'n. State.

Hereford.
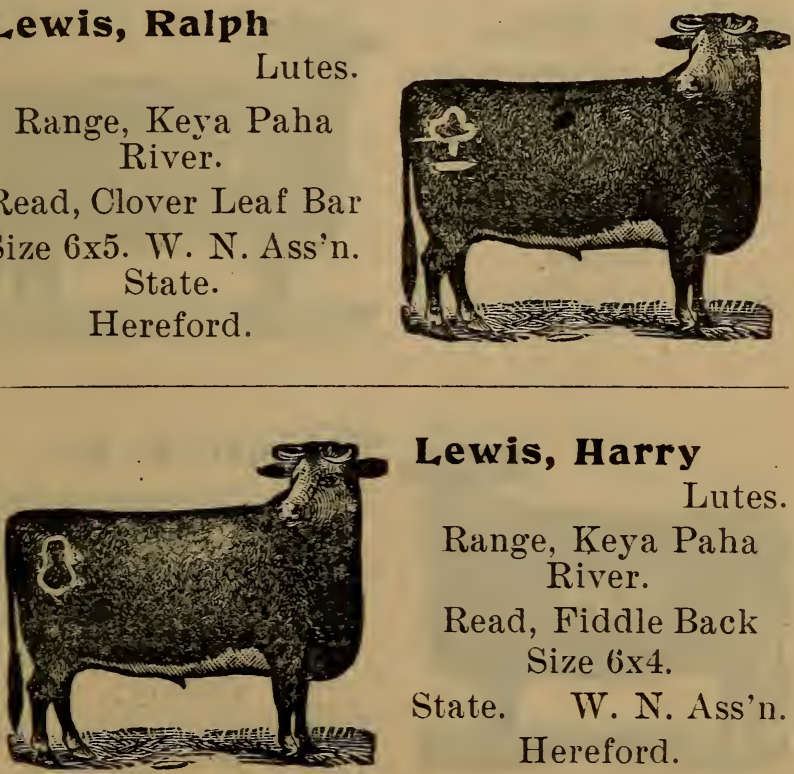

Lewis, Harry

Lutes.

Range, Keya Paha River.

Read, Fiddle Back Size $6 \times 4$.

State. W. N. Ass'n. Hereford. 
Lewis \& Newman

Lutes.

Range, Lutes Creek Read, two half circles bar. On left and hip. Size $5 \times 10$.

Association. Hereford.
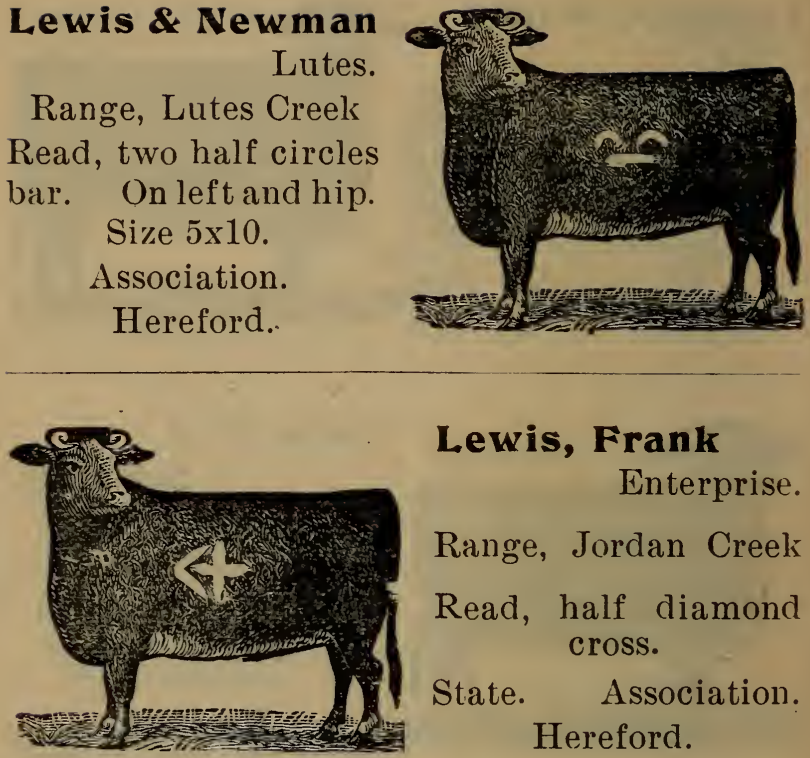

Lewis, Frank

Enterprise.

Range, Jordan Creek

Read, half diamond cross.

State. Association. Hereford.

\section{Munger, Chas.} Springview.

Range, Holt Creek Read, R M Size 5x8 County. Hereford.
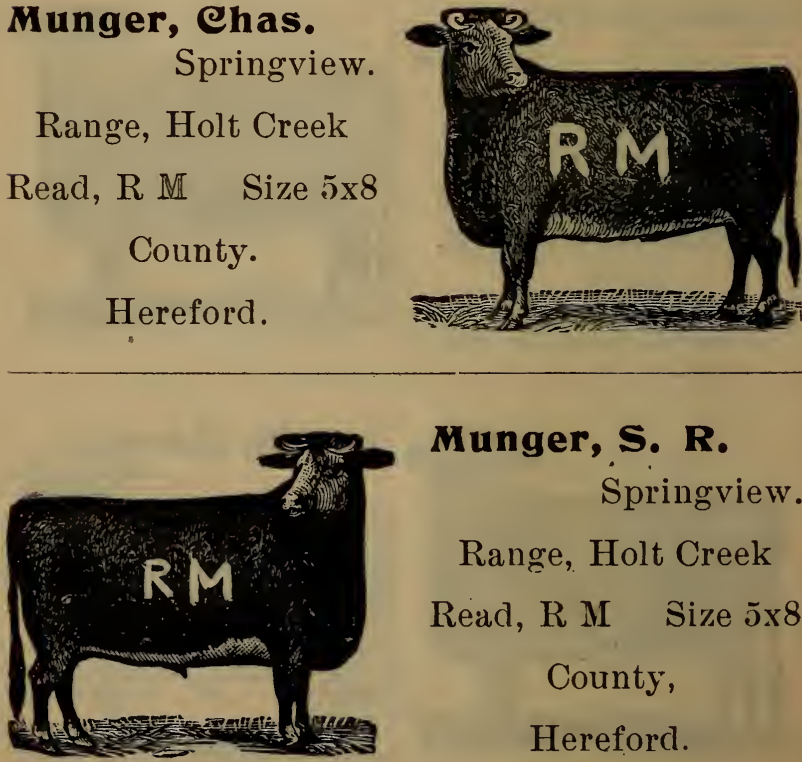

Munger, S. R.

Springview.

Range, Holt Creek

Read, R M Size 5x8 County, Hereford. 


\section{Mays Bros.}

Enterprise.

Rangè, Holt Creek

Read, Gate Size 7 in.

State.

Durham.
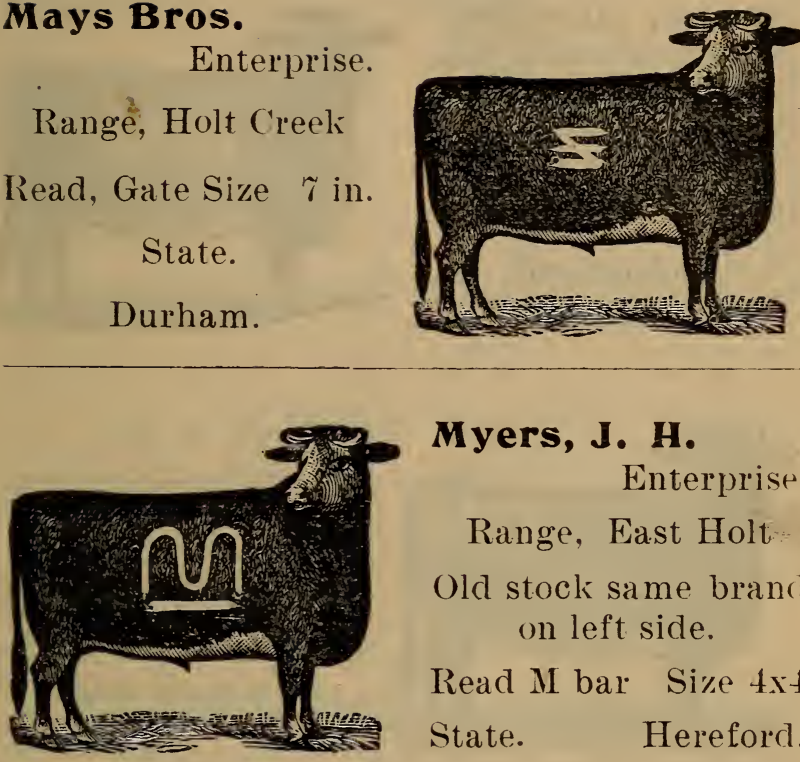

Myers, J. H.

Enterprise.

Range, East Holt

Old stock same brand on left side.

Read II bar Size $4 x 4$ State. Hereford.

\section{Mickey Bros.}

Springview.

Range, Keya Paha River.

HM connect'd on right side and hip and Mc on right hip. Size $5 \times 8$ state. Short Horn.
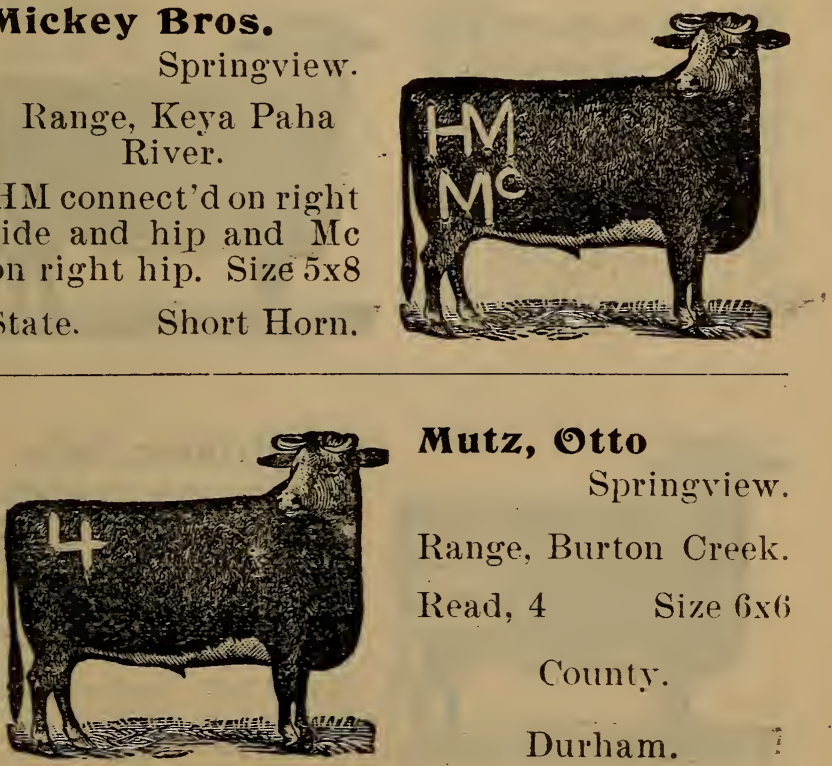

Mutz, Otto

Springriew.

Range, Burton Creek.

Read, 4 Size 6x6

County.

Durham. 
McKee, Herbert

Lutes.

Range, Buffalo Creek Reservation.

Read, H. M. Size $4 \times 10$ Individual

Association. Hereford. .
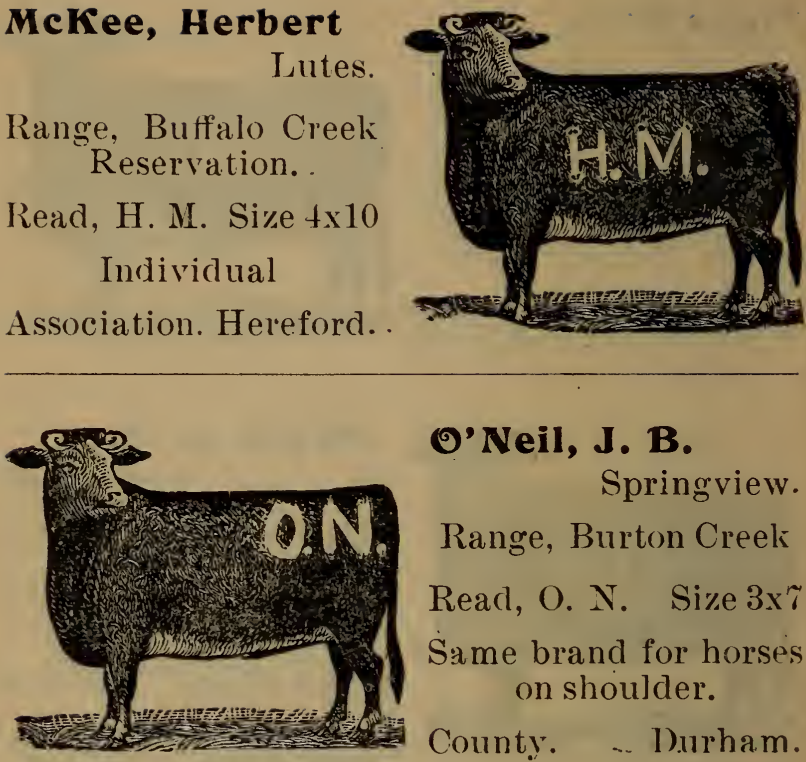

O’Neil, J. B.

Springview.

Range, Burton Creek

Read, O. N. Size $3 x \%$ Same brand for horses on shoulder.

County. _. Durham.

\section{Millikin, Perry \& co}

Springriew,

Range, Spring Creek

Read, $2 \quad$ Size $2 \frac{1}{2} \times 2 \frac{1}{2}$

Individvai.

Mixed.
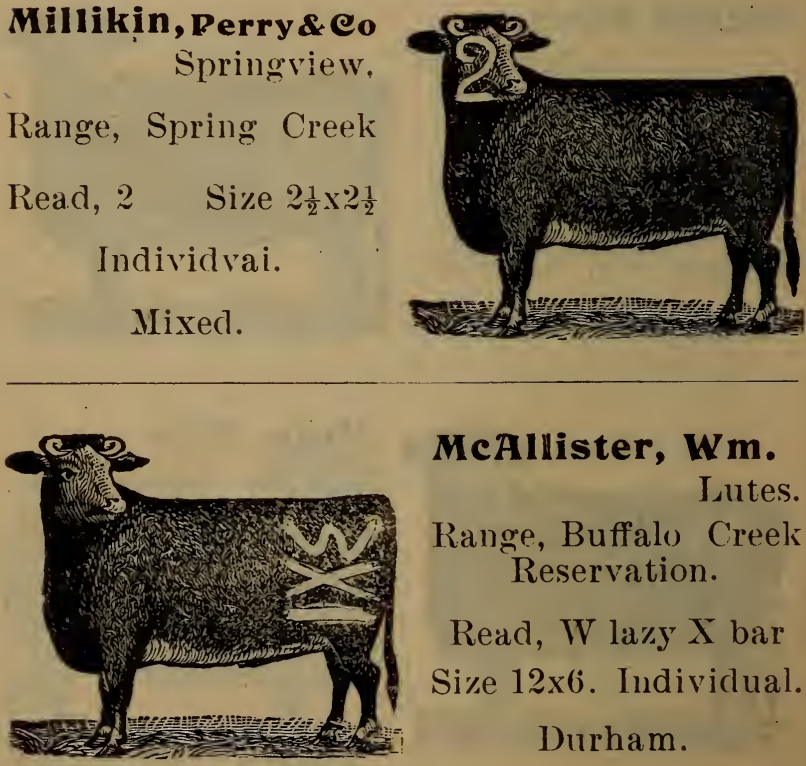

McAllister, Wm. Lutes.

Range, Buffalo Creek Reservation.

Read, WV lazy $\mathrm{X}$ bar Size $12 x 6$. Individual.

Durham. 
Outhouse, A. J.

Springview.

Range, Dutch Flat

Read, Triangle

Individual. Si\%e $3 \times 3$

Durham.
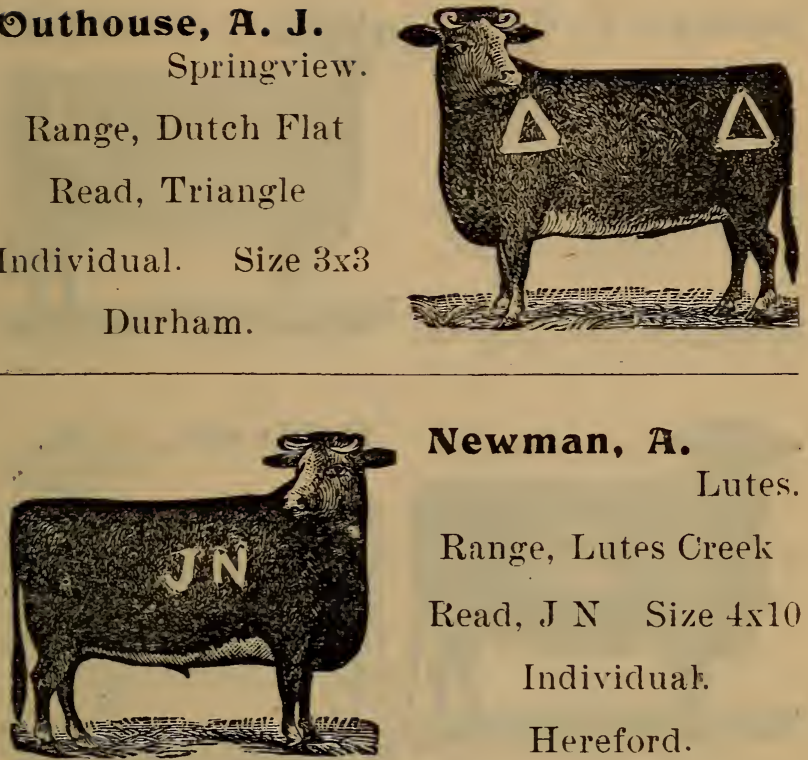

Newman, A.

Lutes.

Range, Lutes Creek

Read, J N Size $4 \times 10$

Individuak.

Hereford.

Palmer, L. W. and G. W. Palmer

Springview.

Range, Spring Creek

Read, 17 bar Size $6 \times 6$

County. Mixed.
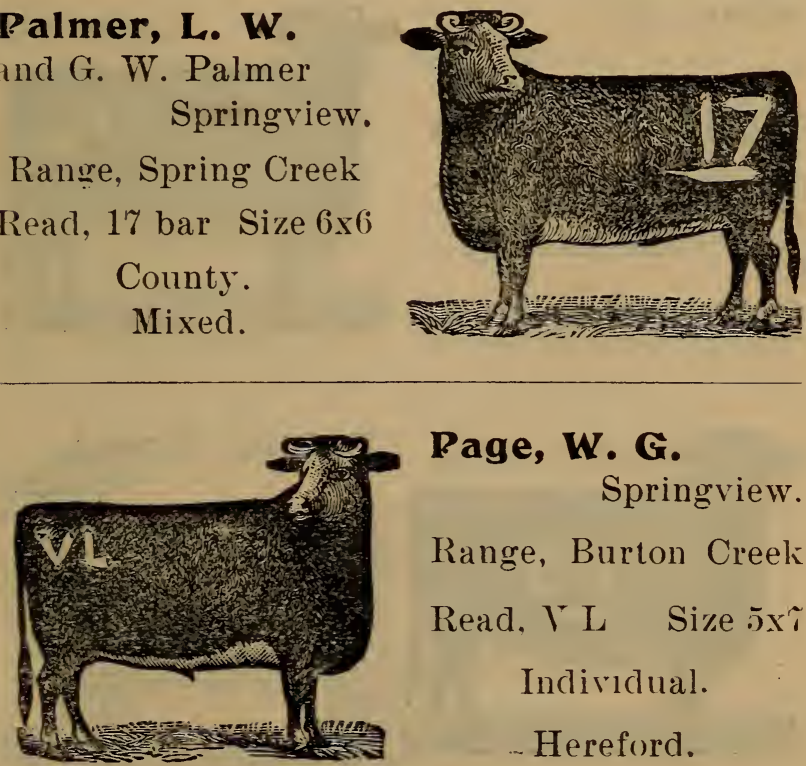

Page, W. G.

Springview.

Range, Burton Creek

Read, I L Size 5xi

Indiridual.

Hereford. 
Rhodes, F. J.

Lutes.

Range, Keya Paha River

Read, Cockeye Size4x2

County.

Hereford.
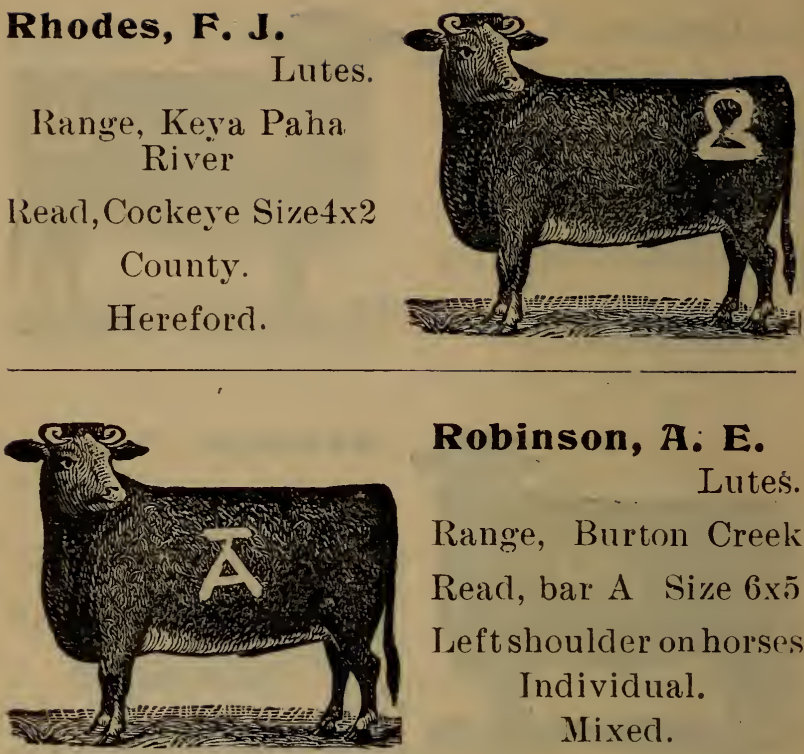

Robinson, A: E.

Luteś.

Range, Burton Creek Read, bar A Size 6x5 Left shoulder on horses Individual. Mixed.

Shaw, A.

Lutes.

Range, Burton Creek Read, A lazy s Size $4 \times 6$

Individual. Nixed.

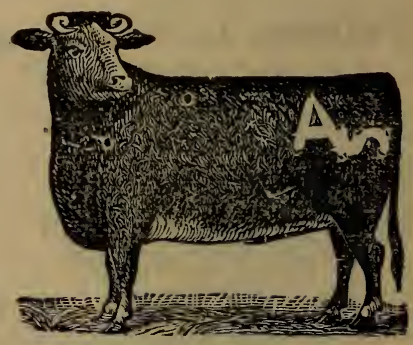

\section{Snider, eyrus}

Lutes.

Range, Iutes Creek Read, Y S bar Size $8 \times 10$

State.

Hereford. 
Snider, Lee

Lutes.

Range, Lutes Creek

Read, Quarter circle

7 bar. Size 10x10

State. Hereford.
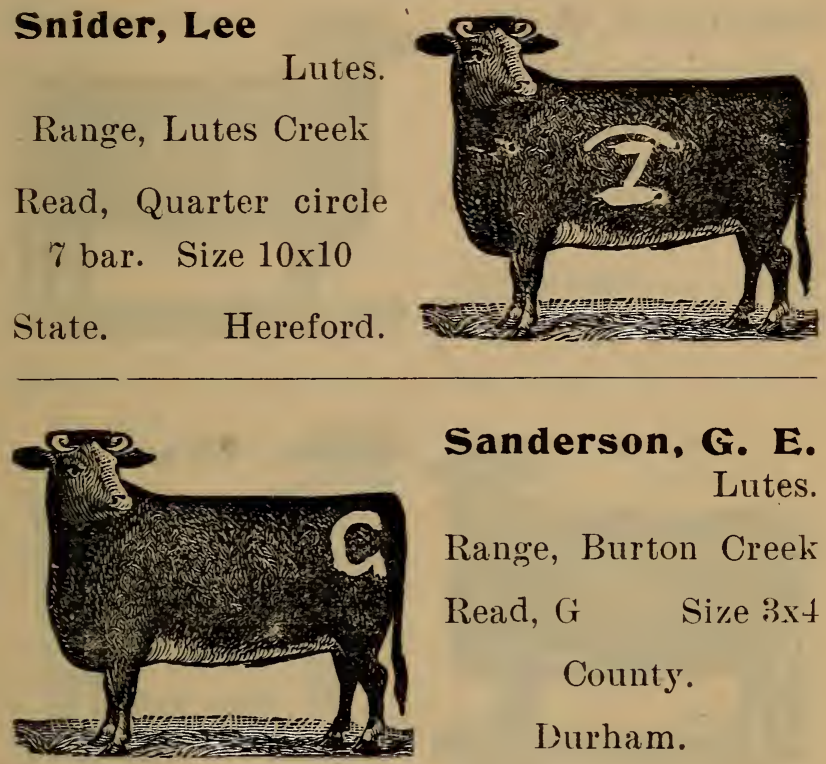

Sanderson, G. E.

Lutes.

Range, Burton Creek

Read, G Size $3 \times 4$

County.

Durham.

\section{Sylvester,}

W. B. and $A$. Lutes.

Range, Buffalo Creek Read, Jewsharp $4 x^{r}$ Horses above left arm County. Association. Durham.

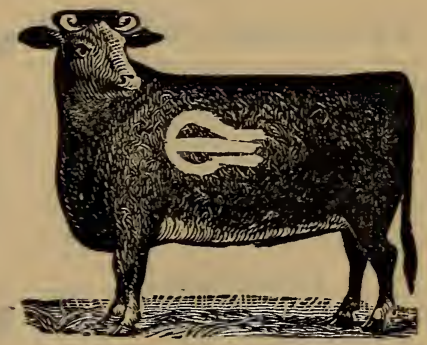

Shaw, Henry e. Mills.

Range, 2 miles north of Spring Creek Read, S bar C Size $4 \mathrm{x}$ \% County. Hereford. 
Stapleton, C. A. Mills.

Range, Keya Paha River

Read, C 7

State. Association. Durham.
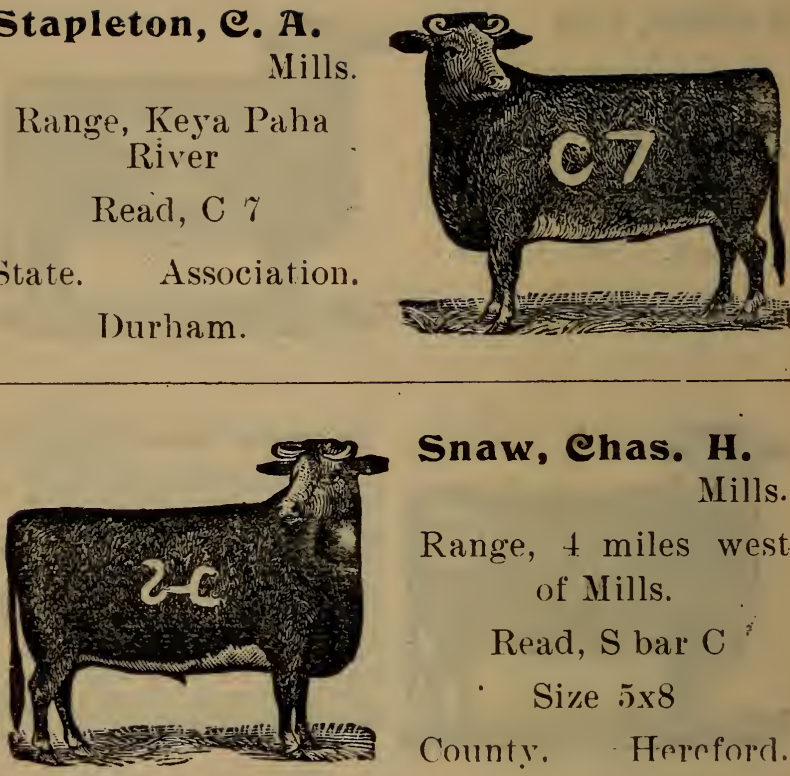

\section{Snaw, Chas. H.} Nills.

Range, 4 miles west of Mills.

Read, $\mathrm{S}$ bar C

- Size 5x8

County. Hereford.

Sylvester, Chas. Nills.

Range, Keya Paha River

Read, Ace of Hearts and Ace of Hearts bar Individual. Durham.

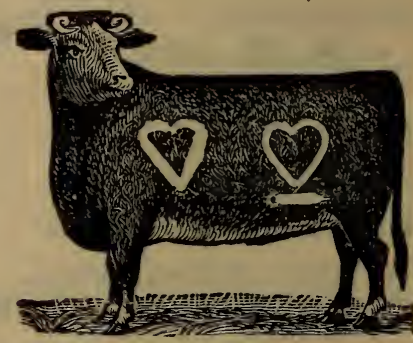

Sprague, G. W. Mills.

Range, Buffalo Creek Read, O T Size $6 \times 6$ Individual. Roan Durham. 
Schoattger, Fred

Enterprise.

Range, Holt Creek

Read, F S Size $8 \times 8$

Association. State.

Hereford.
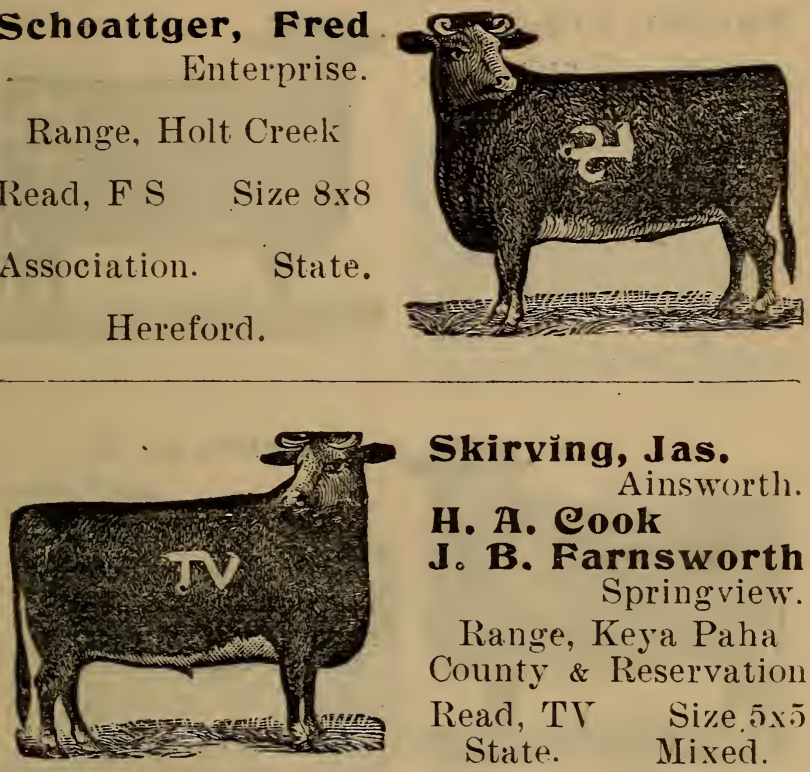

Skirving, Jas.

H. A. Cook

Ainsworth.

J.B. Farnsworth Springview. Range, Keya Paha County \& Reservation Read, TV

Size.5x State. Mixed.

\section{Smith, T.V.}

Springview.

Range, Burton Creek Read, $T$ S Size $4 \times 8$ County.

Hereford.
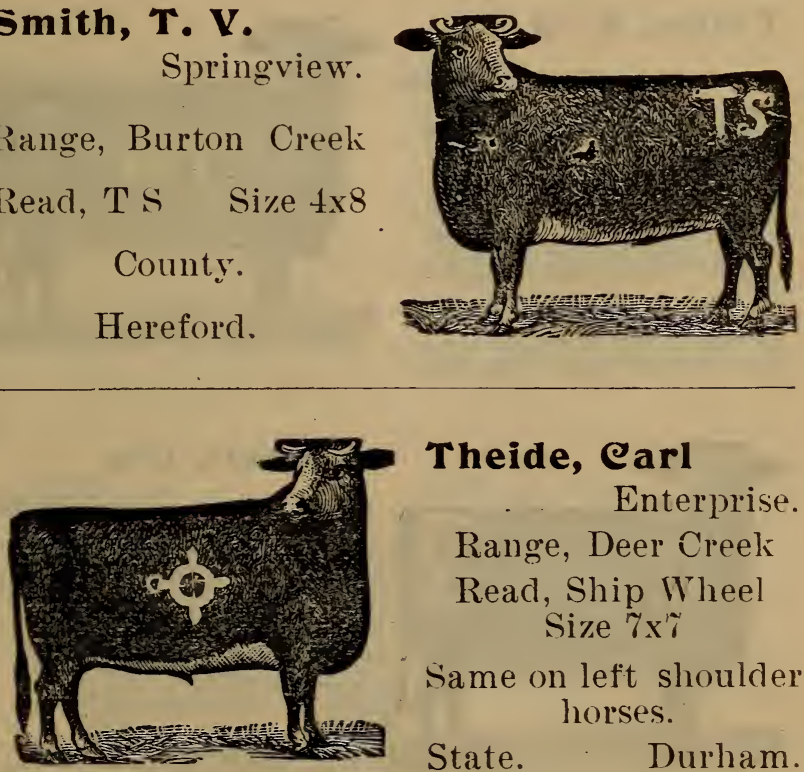

Theide, Carl

Enterprise.

Range, Deer Creek Read, Ship Wheel Size $7 x^{2} \%$

Same on left shoulder horses.

State. Durham. 


\section{Theide, Fred}

Enterprise.

Range, Dutch Flat

Read, Ship Wheel

Size $5 \times 5$.

State.

Durham.
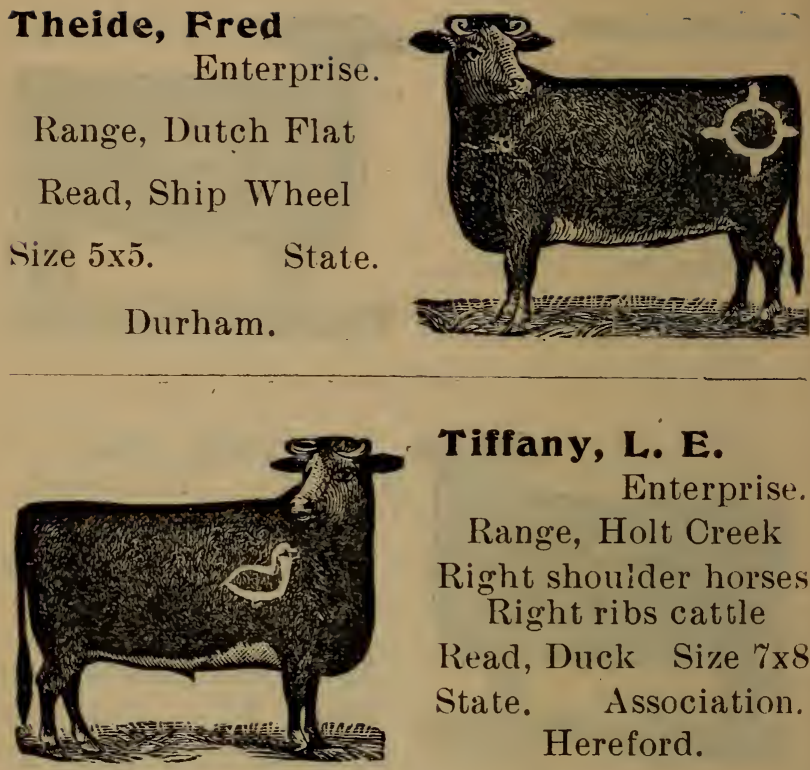

Tiffany, L. E.

Enterprise.

Range, Holt Creek

Right shoulder horses

Right ribs cattle

Read, Duck Size $7 \times 8$

State. Association. Hereford.

Tuton, N. B.

Range, Keya Paha River.

Read, U N Size $4 \mathrm{x} \tilde{y}$

State. Association.

Mixed.

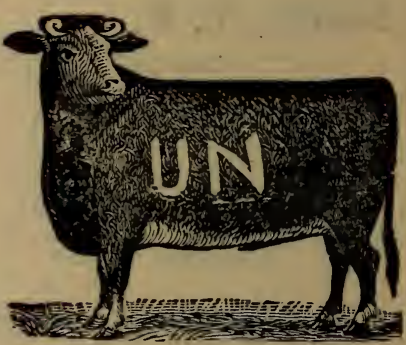

\section{Verrell, Dix}

Enterprise.

Range, Họlt Creek

Read, bar $\mathrm{Y}$ bar

Size $4 \mathrm{x} 4$

Individủal.

Hereford. 
Wiley, Ed

Range, Burton Creek Read, J IT Size $3 x$ ?

\section{County.}

Durham.
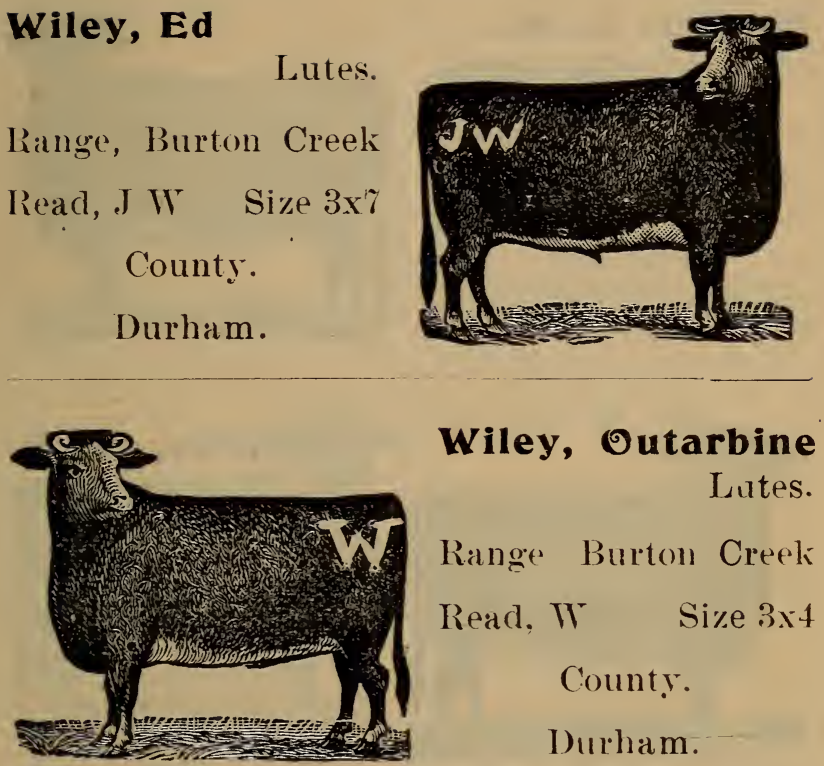

Wiley, Outarbine Lutes.

Range Burton Creek Read, II Size $3 \times 4$ County. Durham.

Wiley, Wm.

Lutes. Range, Burton Creek Read, $\mathrm{L} \mathrm{X}$ bar Size $8 \times 12$

Horse brand JW bar on left shoulder Ass'n. Individual. Hereford.

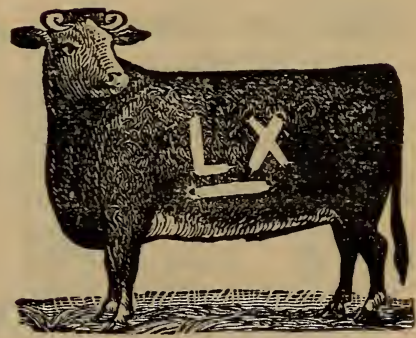

\section{Wiley, Jas.}

Lutes.

Range, Burton Creek Read, JIV Size 3xi

County.

Durham. 
Warner, W. W.

Enterprise.

Range, Holt Creek

Read, Y inverted

Size $3 x 2$

Individual. Hereford.
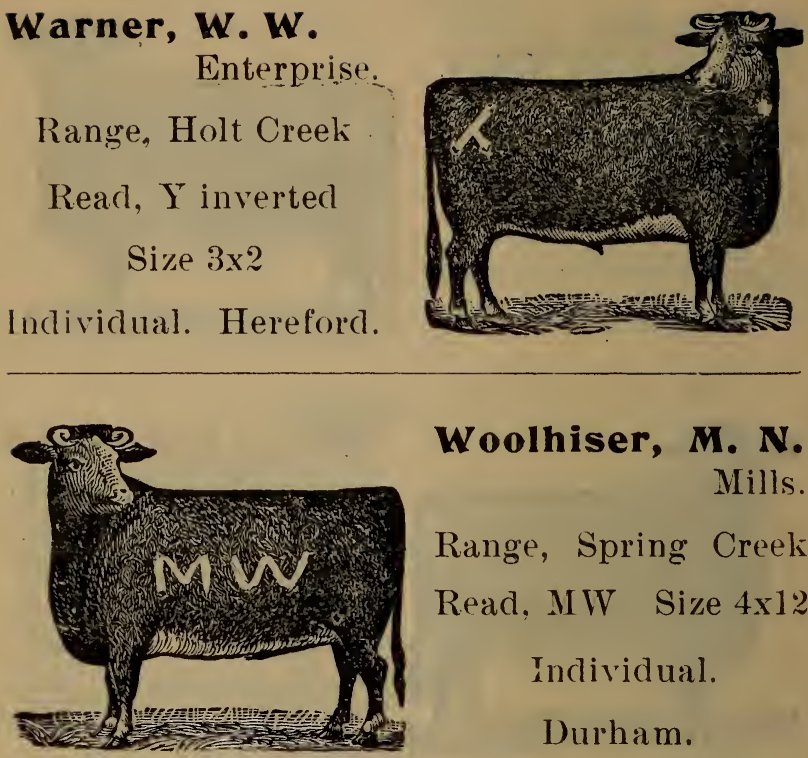

Woolhiser, M. N.

Mills.

Range, Spring Creek

Read, MIV Size $4 \times 12$

Individual.

Durham.

Webster, Chas. Enterprise.

: Range, Holt Creek

Read, Pump Size 10x8

Siate. Association.

Hereford.

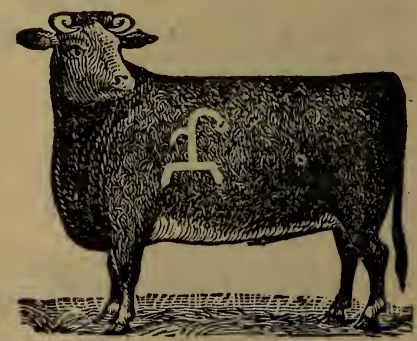

Webster, e. $\boldsymbol{0}$.

Enterprise.

Range. Holt Cre $€$ k

Read, Shield Size 8x.5

Right shoulder on horses.

Association. State.

Hereford. 
Woolhiser, A.

Mills.

Range, Spring Creek Read, AW Size $4 \times 6$ Individual. Durham.
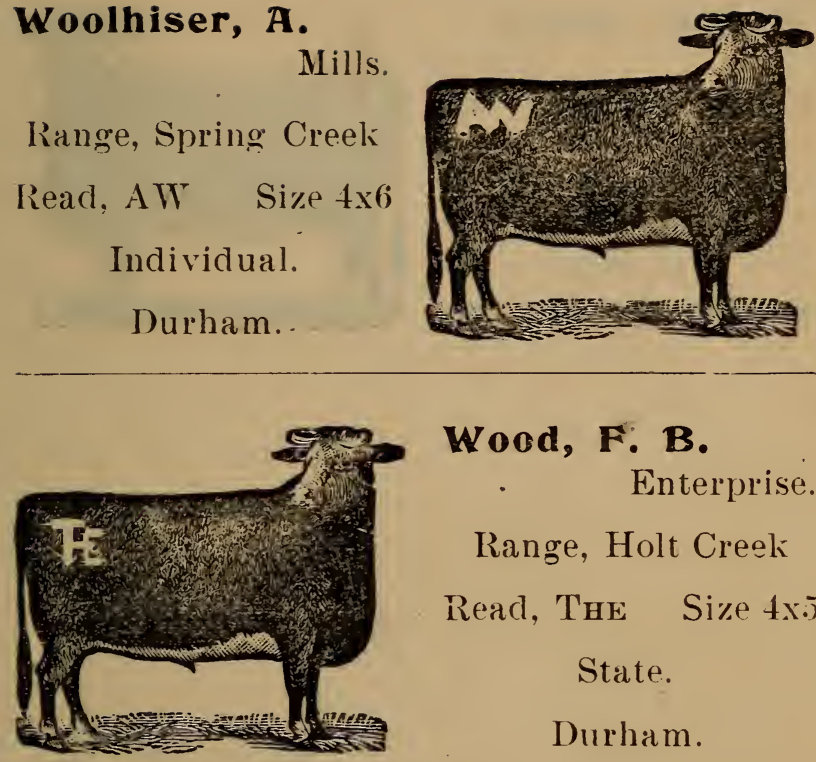

Wood, F: B.

Enterprise.

Range, Holt Creek

Read, The Size 4x.j

State.

Durham.

Wood, M. M.

Range, Holt Creek

Read, Box Bar on right front leg.

Bar 4 in. Box 5x5 Individual.
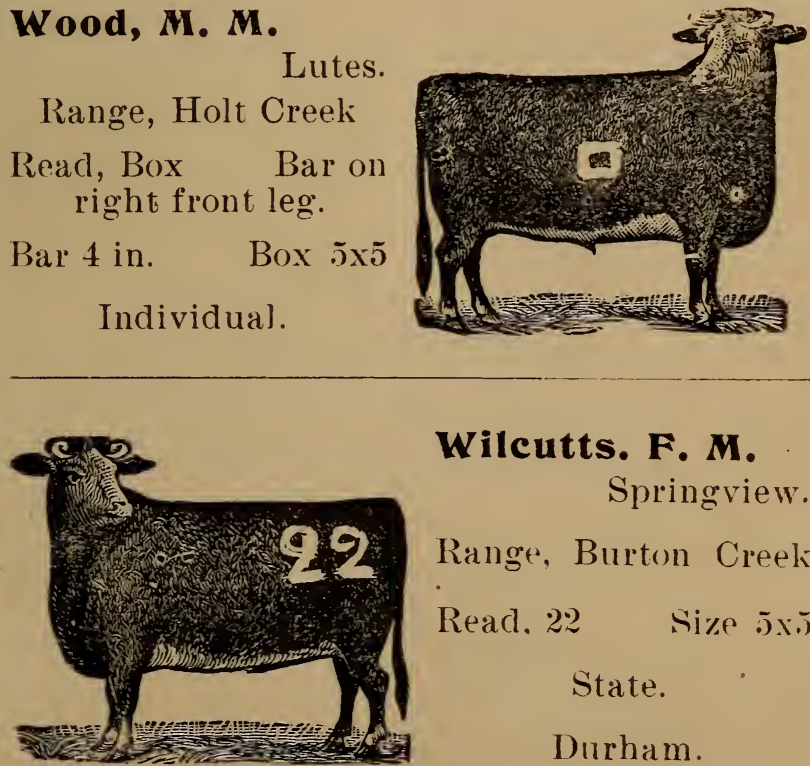

Wilcutts. F. M.

Springview.

Range, Burton Creek Read. 22 Nize 5x.

State.

Durham. 
36

\section{Woods, John P.}

Lutes.

Range, $2 \frac{1}{2}$ miles east of Lutes.

Read, Half Circle () Size $4 \times 8$

County.

Durham, Polled Angus

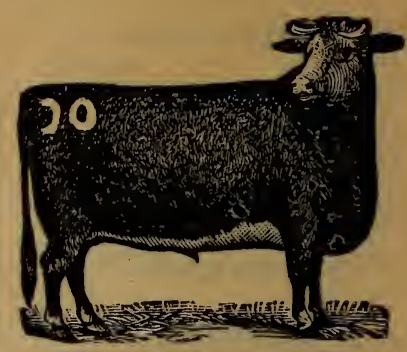





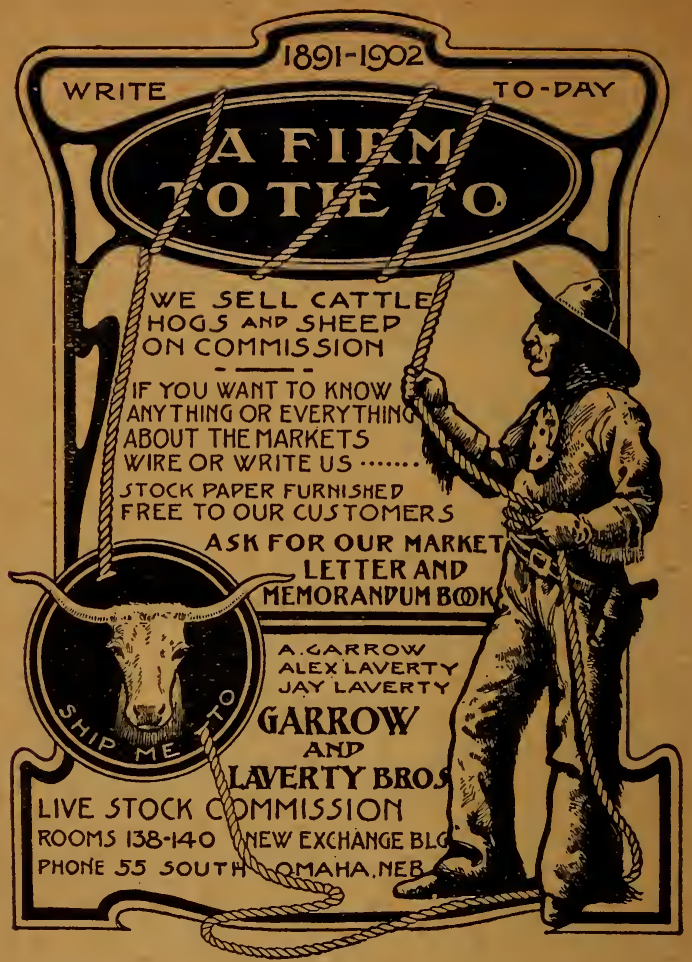

We solicit your trade and assure you that whatever stock you send us will be-cared for properly, sold on the the market and the proceeds remitted to you promptly as you direct. As to our responsibility, we refer by permission to the Union Stock Yards National Bank, or to the Union Stock.Yards Company here.

We do a strictly commission business, selling Cattle, Hogs and Sheep consigned to us and buying on orders. We furnish any kind of stock wanted by our customers and any inquiries you make will have our prompt attention and you will be given all the information we have at hand. We treat every one alike-ON THE SQUARE-and the shipper of one car or of fifty gets just the same attention. You are cordially invited to call upon us when you are here, whether you have stock or not, and get acquainted with us and our methols of doing business. We feel sure that any one who gives us a trial will be our customer afterwards.

Our salesmen are experienced men. all having been in the stock business for years, and we risk our reputation on the assertion that there are no better anywhere. In short, we promise you PERSONAL ATTENTION to all stock consigned to us, and feel confident that we are prepared in every way to give you the best of service. WRITE US. WIRE US. SHIP US. Yours very truly,

GARROW \& LAVERTY BROS. Rooms 138=140 New Exchange Building Phone 55. South Omaha, Nebraska. 


\section{Custer Precinct.}

\section{Allen, S. S.}

Springview.

Range, Springview Table

Read, S H A Size $4 x$ '

siame on loft shoulder herese:s.
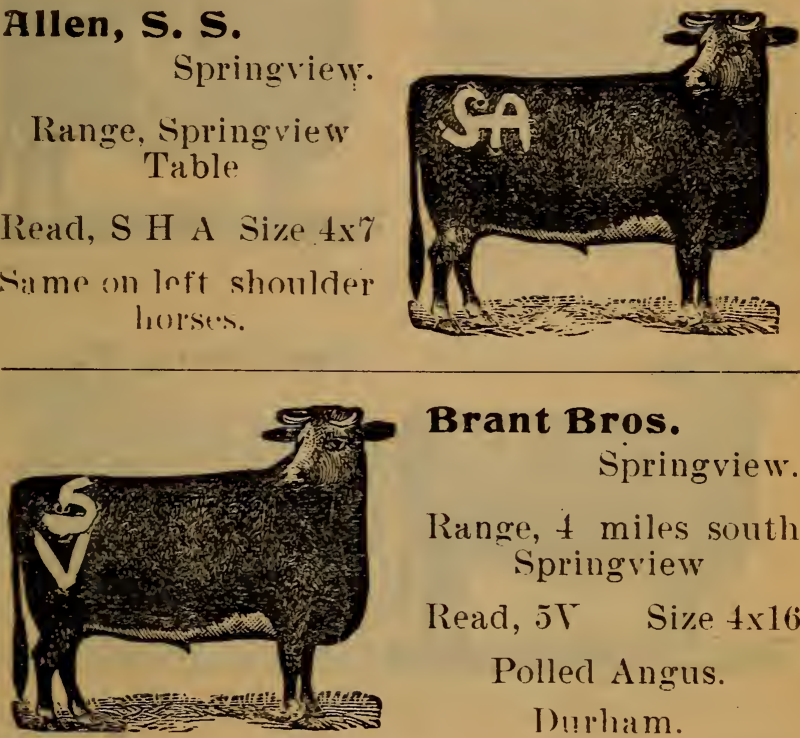

\section{Brant Bros.}

Springview.

Range, 4 miles south Springview

Read, 5 T Size $4 \times 16$

Polled Angus.

I) turham.

\section{Ballard, Geo.}

Springriew.

Range, Darnall Valley

Read, B 3 . Size 4x9

Association." State.

Herefords.
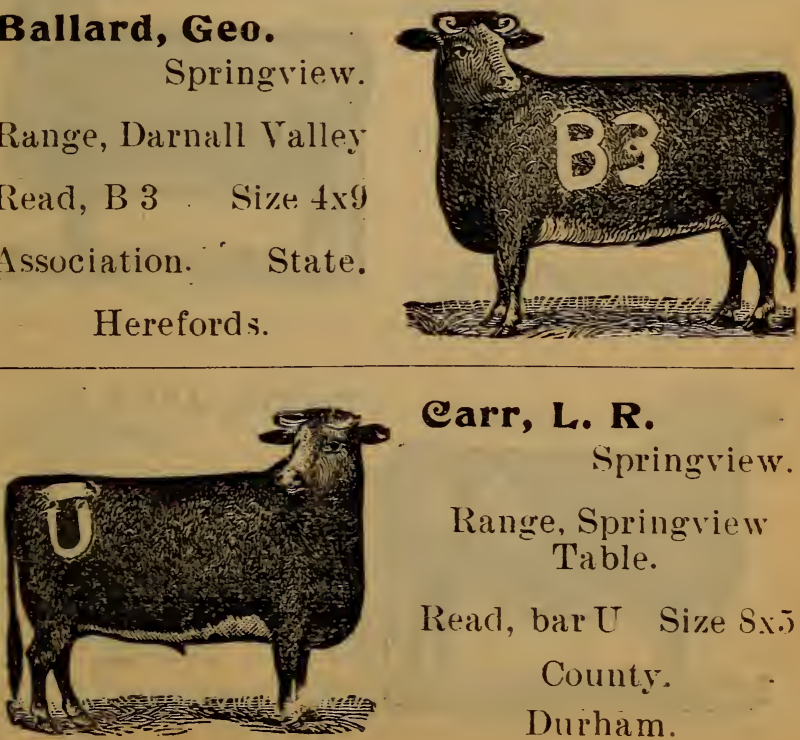

\section{Carr, L. $\mathbf{R}$.}

Springriew.

Range, Springview Table.

Read, bar U Size Sx.j

\section{County.}

Durham. 
Carnahan, J. S.

Springview.

Range, 6 miles south of Springview

Read, U O Size $6 \times 2 \frac{1}{2}$ County. Galoway.
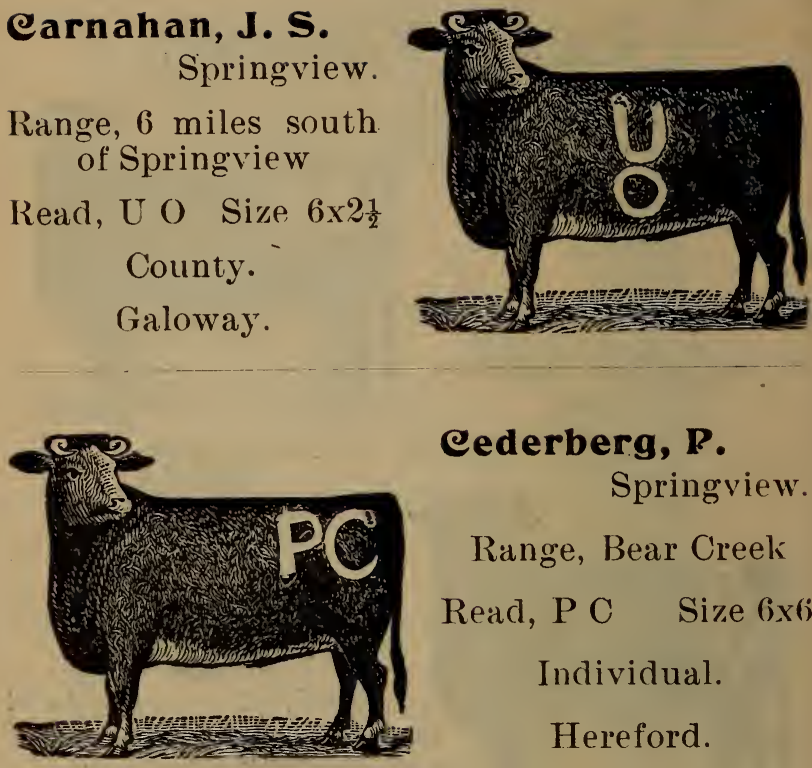

\section{cederberg, $\boldsymbol{P}$.}

Springview.

Range, Bear Creek

Read, P C Size 6x6

Individual.

Hereford.

\section{Carr, D. $M$.}

Springview.

Range, Iowa table

Read, I) C Size 5x12

County.

Durham.

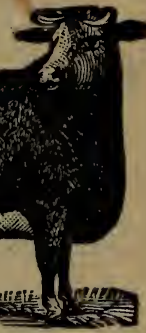

\section{Coble, John $M$.}

Springview.

Range, Dry Valley

Read, Diamond $\mathbf{X}$

Size $5 \times 10$

State.

Mixed. 
Carr, John F.

Springview.

Range, Springview Read, U P Size 6x\% State.

Durham.

Association.

Horses on left shoulder
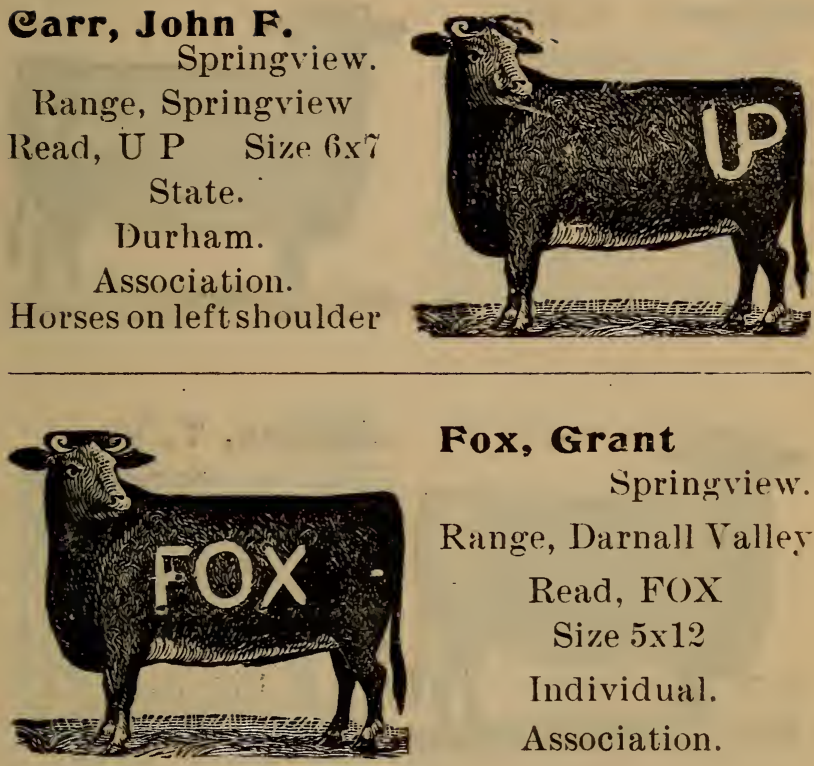

\section{Fox, Grant}

Springriew.

Range, Darnall Valley

Read, F()X

Size $5 \times 12$

Individual.

Association.

\section{Hollock, Elen F.}

Springview.

Range, Springview Table.

Read, R I Size 5x10

County.

Mixed.
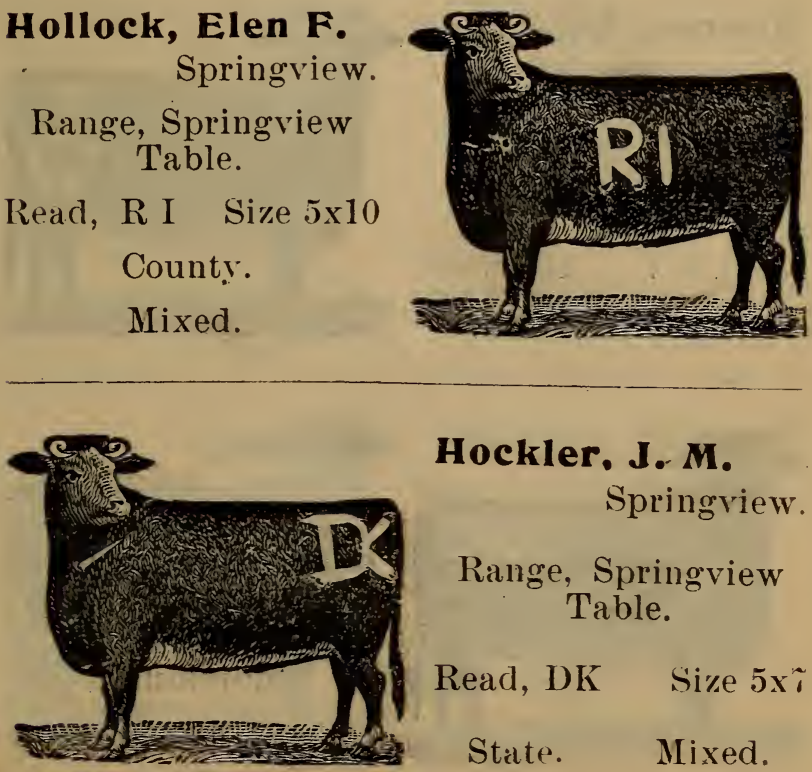

Hockler, J. M.

Springriew.

Range, Springview Table.

Read, DK Size 5xi

State. Mixed. 
Johnson, John

Springview.

Range, Prosser Creek Read, J J Size 6x6

Individual .

Hereford.
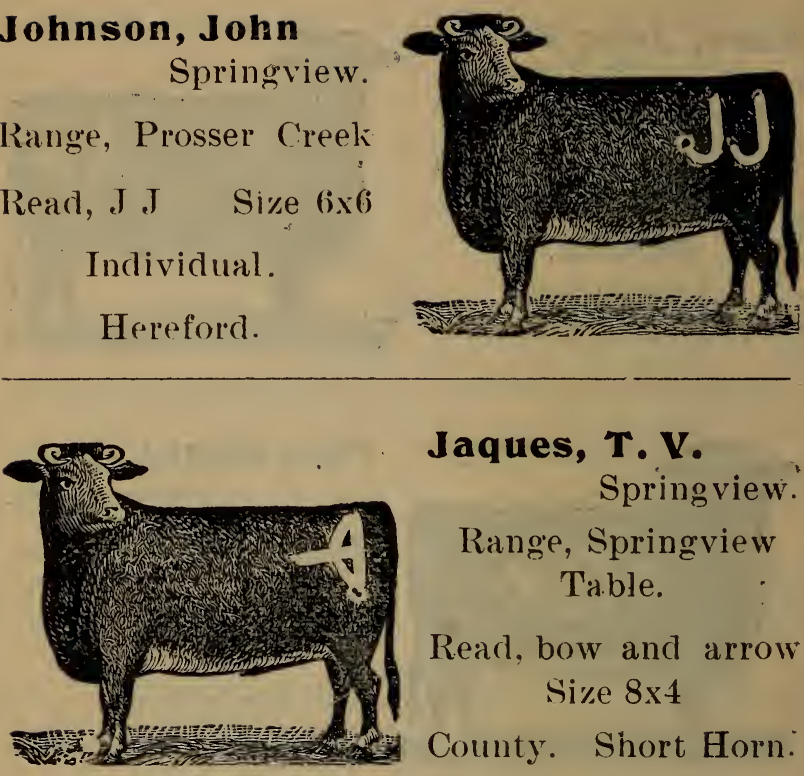

Jaques, $\mathbf{T}$. V.

Springview.

Range, Springview

Table.

Read, bow and arrow

Size $8 \times 4$

County. Short Horn:

\section{Monroe, Ed.}

Springview.

Range, 6 miles south of Springview and Reservation.

Read, $2 \mathrm{~V} \quad$ Size $8 \times 8$ State. Hereford.
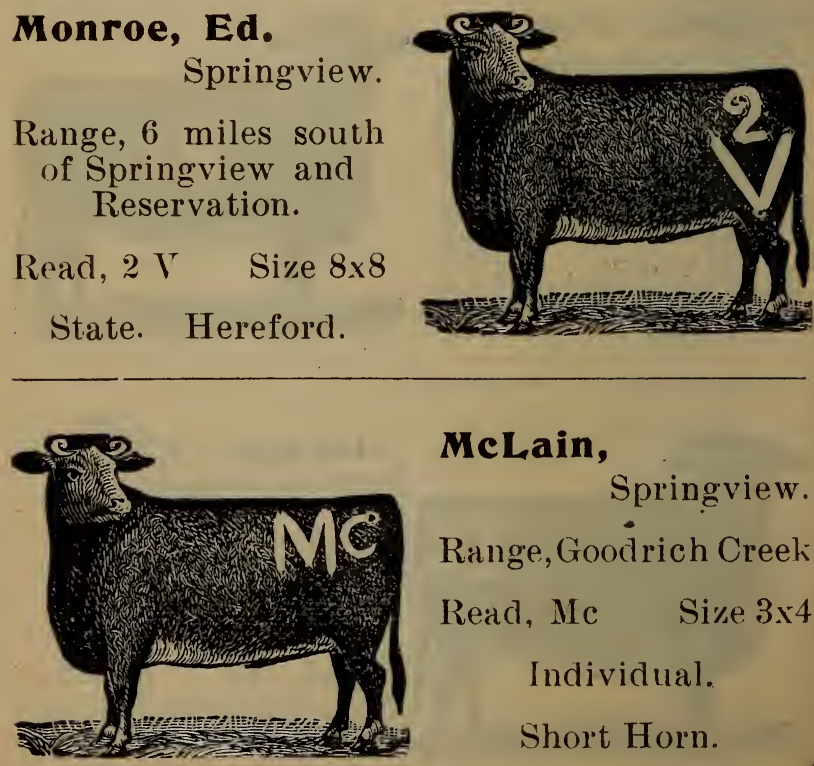

\section{MeLain,}

Springview.

Range, Goodrich Creek

Read, Mc Size $3 \times 4$

Individual.

Short Horn. 
Painter, B. A.

Springriew.

Range, 3 miles southwest Springview

liead, I, P size.xs

Individual.

Durham.
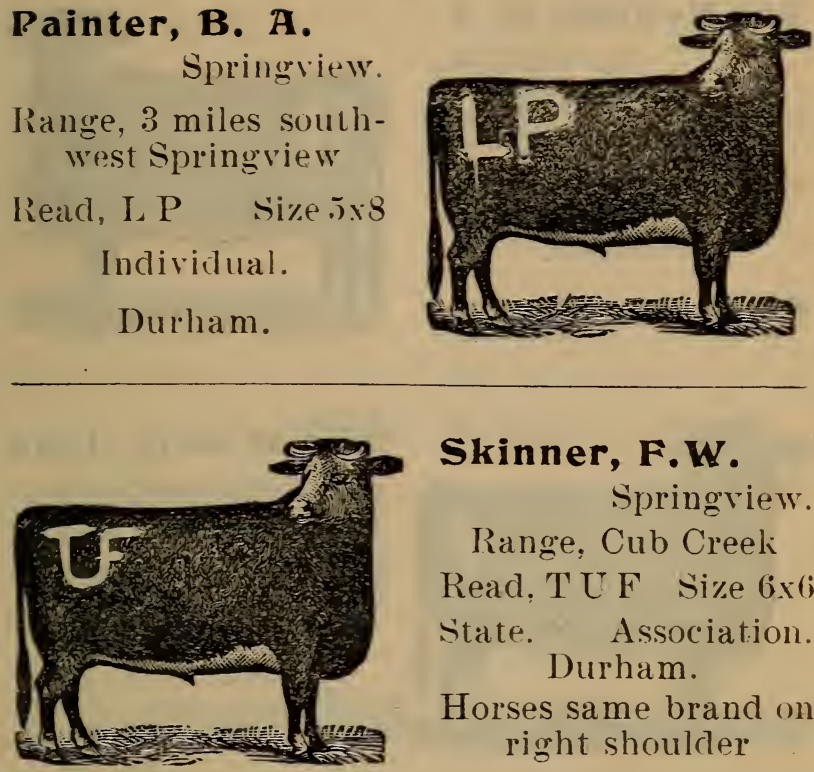

Skinner, F.W.

Springview.

Range, Cub Creek

Read, T U F size $6 \times 16$

state.

Association.

Durham.

Horses same brand on right shoulder

Skinner, Thos.

Springview.

Range, Skinner Creek

Read, circle T S Size 8x $\tau$

Individual.

Hereford.
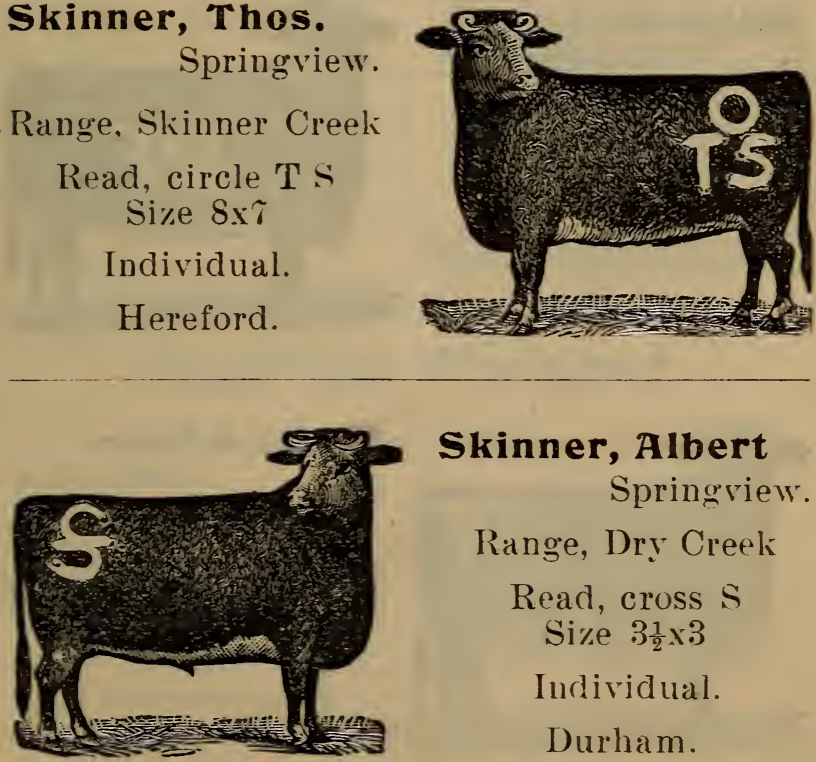

Skinner, Albert

Springriew.

Range, Dry Creek

Read, cross $S$

Size $3 \frac{1}{2} \times 3$

Individual.

Durham. 
Van Houten, e. J. Springview.

Range, Hassett Creek Read, $\mathrm{H} \quad$ Size $4 \times 3$ Hereford.

Horses on left shoulder
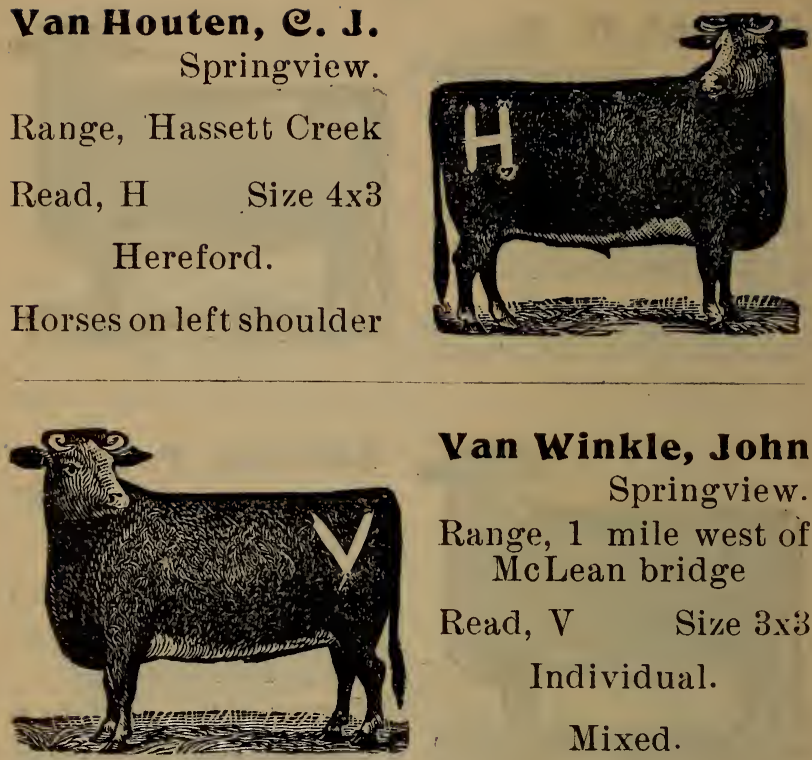

Van Winkle, John Springview. Range, 1 mile west of McLean bridge

Read, V Size $3 \times 3$ Individual. Mixed.

Wolfe, e. N.

Springview.

Range, Keya Paha river

Read, R F Size $5 \frac{1}{2} \times 12$

Individual.

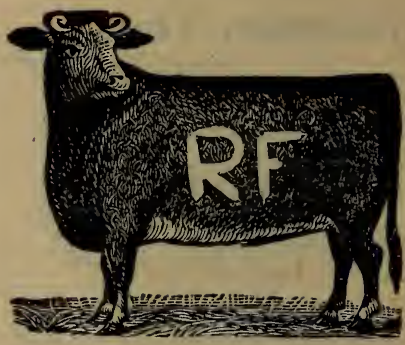

\section{White \& Sons}

Springview.

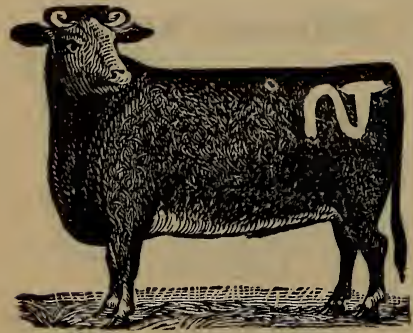

- Range, Iowa Settlement

Read, N T Size $4 \times 4$

Individual.

Mixed.

Same position on horses 
Walls, M. G.

Springview.

Range, Bear Creek

Read, Hat Size $6 \mathbf{6} \mathbf{6}$

State. Durham.

Right shoulder for

horses and mules
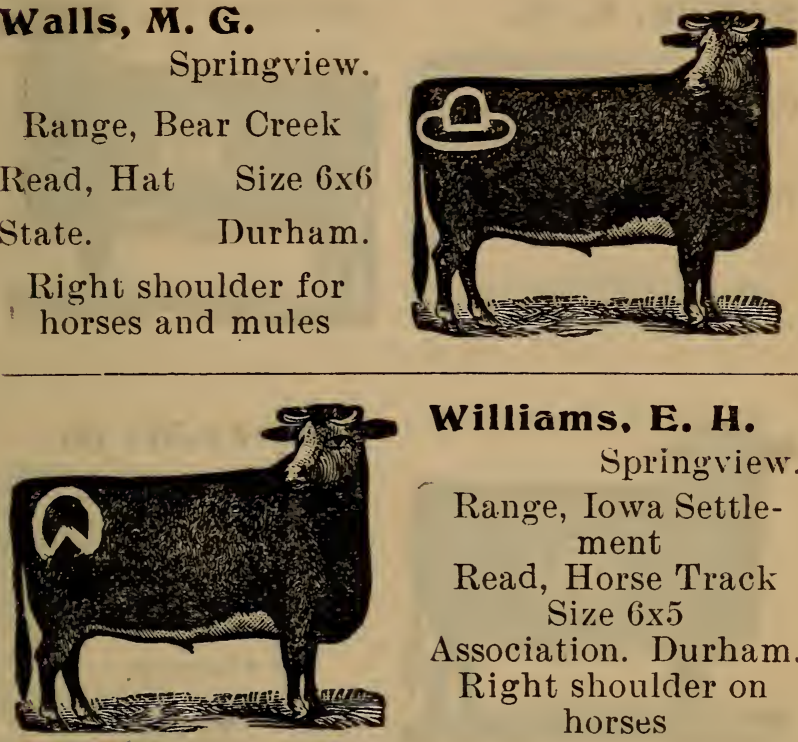

Williams, E. H.

Springview.

Range, Iowa Settlement

Read, Horse Track Size $6 \times 5$

Association. Durham. Right shoulder on horses

\section{Thompson, H. E.} Springview.

Read, $\mathrm{T}$

Individual.

Polled Angus.

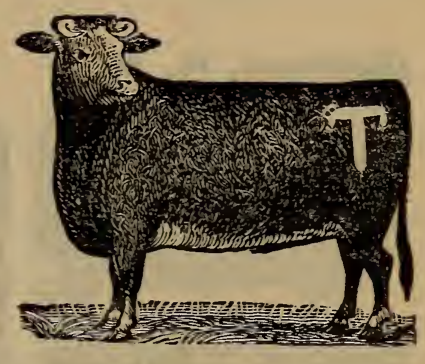

\section{Garfield Precinct.}

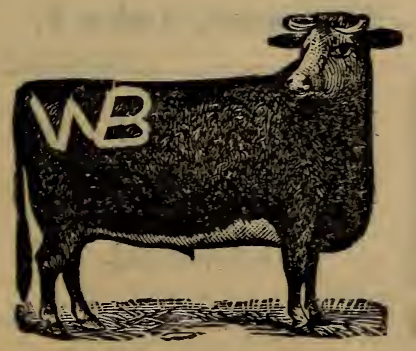

Blake, Wm.

Springriew.

Range, between Cub and Rock Creeks

Read, WB connected State.

Horses right shoulder 
Barton, R. A.

Springview.

Range, near the mouth of Rock Creek

Read, rHL connected Size $4 x^{r} z$

County. Hereford.
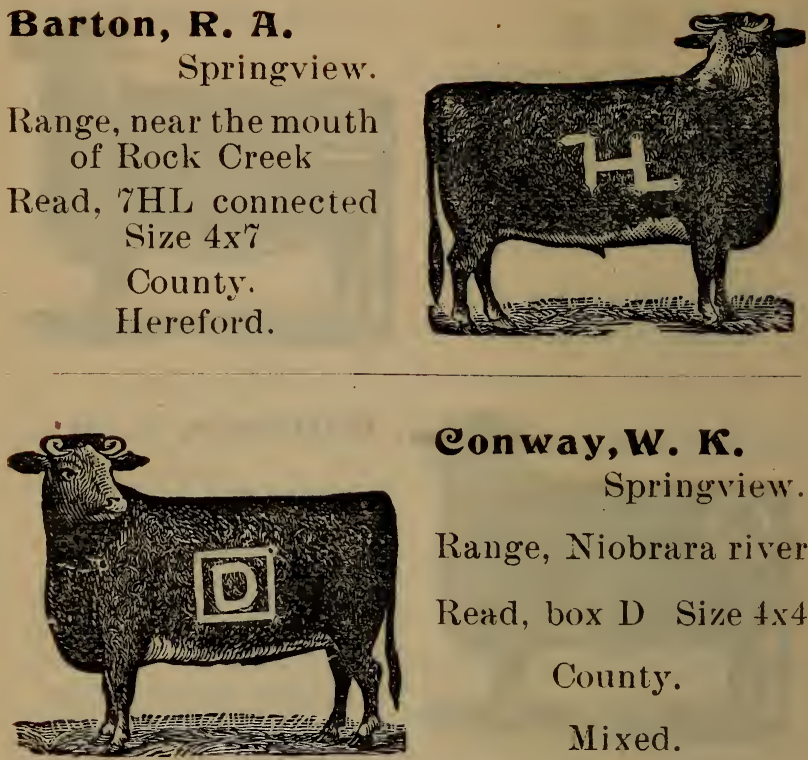

Conway, w. K.

Springview.

Range, Niobrara river

Read, box D Size $4 \times 4$

County.

Mixed.

Dawson, H. M.

Springview.

Range, Chimney creek and Niobrara river Read, quarter circle D Si 6 6 6

Individual.

Hereford. Durham.
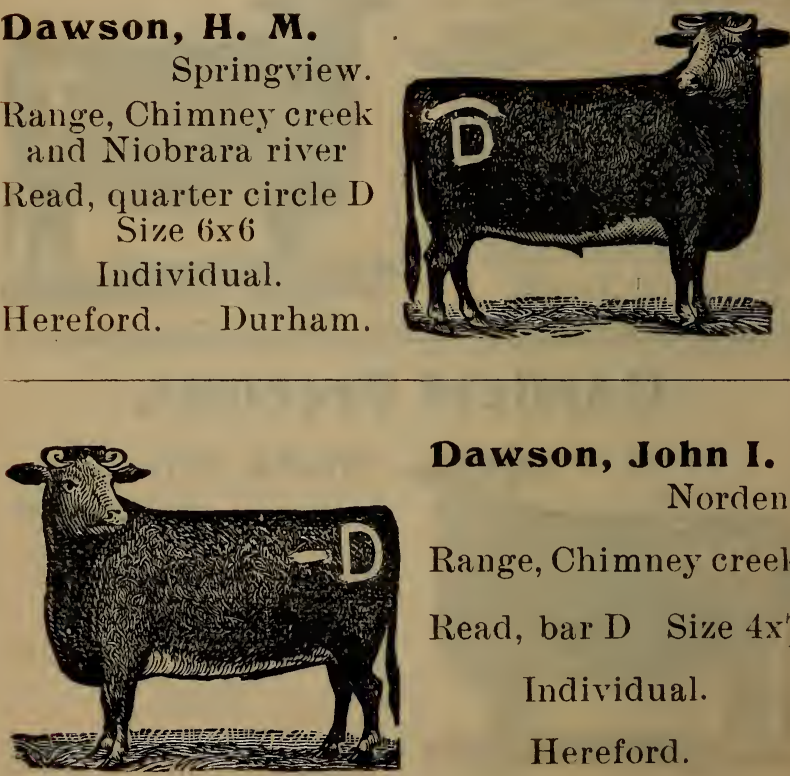

Dawson, John I.

Norden.

Range, Chimney creek

Read, bar D Size $4 x^{\varkappa}$

Individual.

Hereford. 
Foster, Josephine Springview.

Range, Jewett Creek

Read, J J Size $4 \times 4$

Durham.
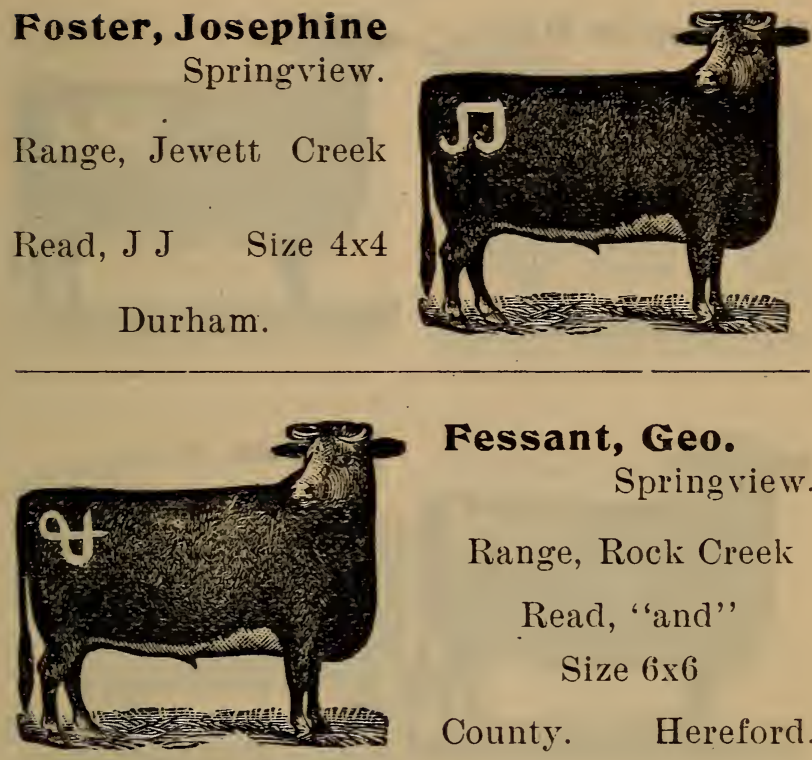

Fessant, Geo.

Springriew.

Range, Rock Creek

Read, "and"

Size $6 \times 6$

County. Hereford.

Greenough, G. F.

Springview.

Range, Rock Creek

Read, C F Large

Mixed.
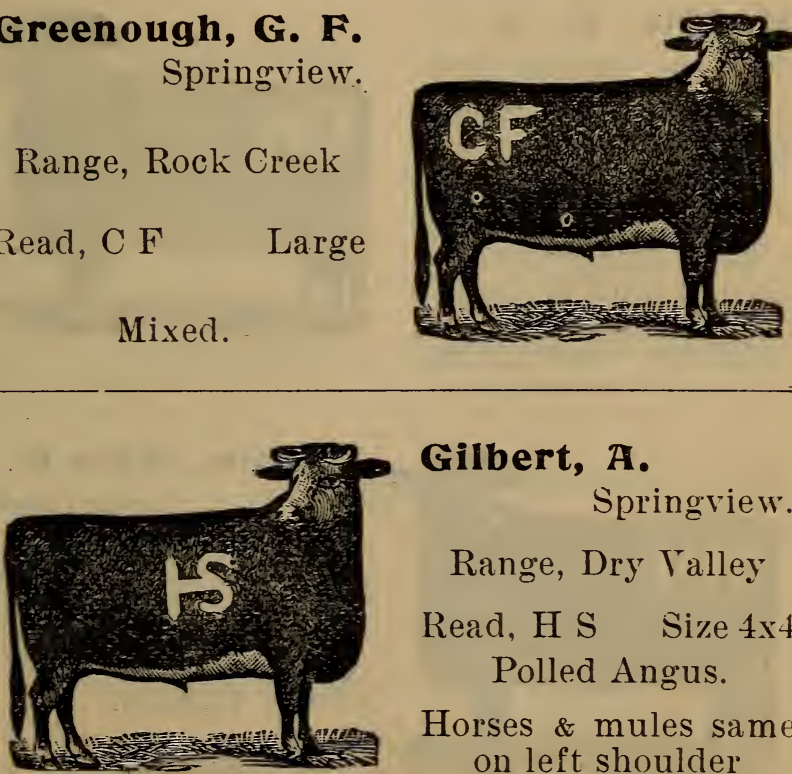

Gilbert, $A$.

Springriew.

Range, Dry Valley

Read, H S Size 4x4

Polled Angus.

Horses \& mules same on left shoulder 
Henneman Bros.

Springview.

Range, Jewett Creek

Read, H M connected Size $4 \mathrm{x}^{r} \mathrm{r}$

State.

Durham.
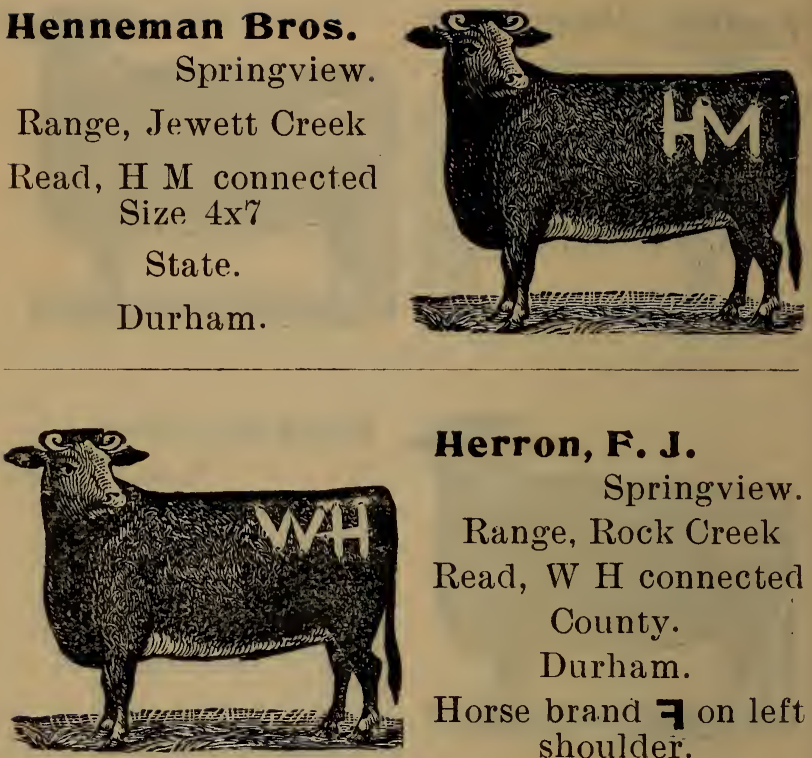

Herron, F. J.

Springview.

Range, Rock Creek

Read, W H connected

County.

Durham.

Horse brand 7 on left shoulder.

\section{Huddle, e. S.}

Springview.

Range, Cub Creek

Read, CH Size $3 \times 6$

County.

Mixed.

Same brand on horses right shoulder.

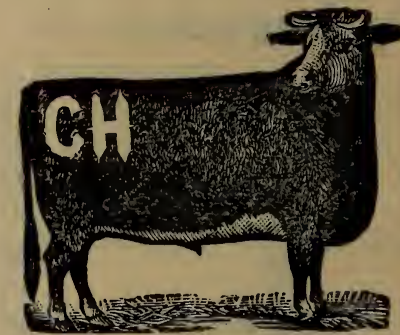

Herron, Mary K.

Springview.

Range, Rock Creek

Read, M H Small

County.

Mixed.. 
Lampitt, Wm.

Springview.

Range, Jewett Creek

Read, W L Size 5x8

Individual.

Hereford.
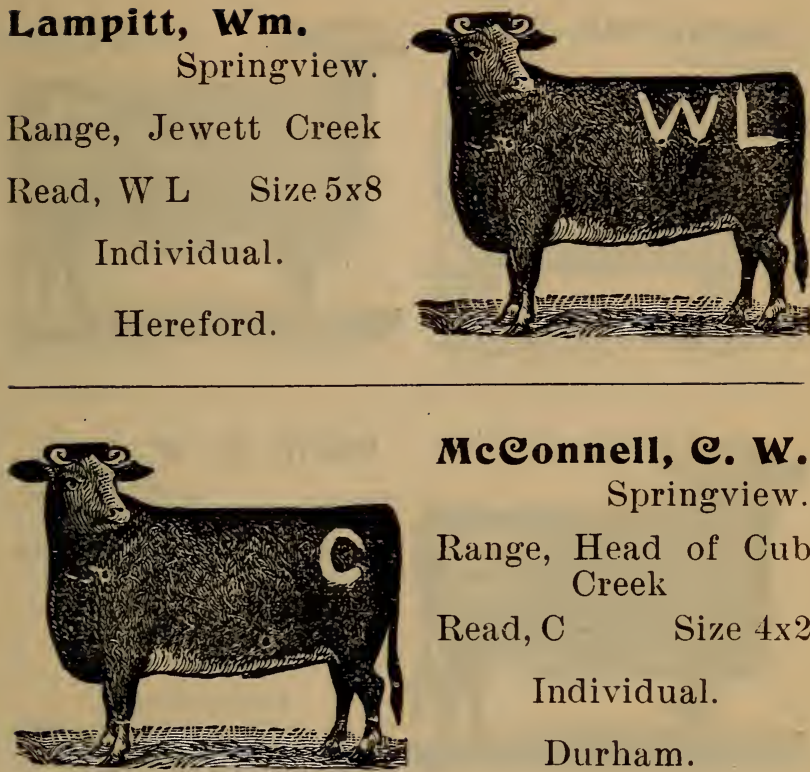

Mceonnell, e. W. Springview.

Range, Head of Cub Creek

Read, C Size $4 \times 2$

Individual.

Durham.

Mceonnell, Chas. Springview.

Range, Head of Cub Creek

Read, C

Size $4 \times 2$

Individual.

Mixed.
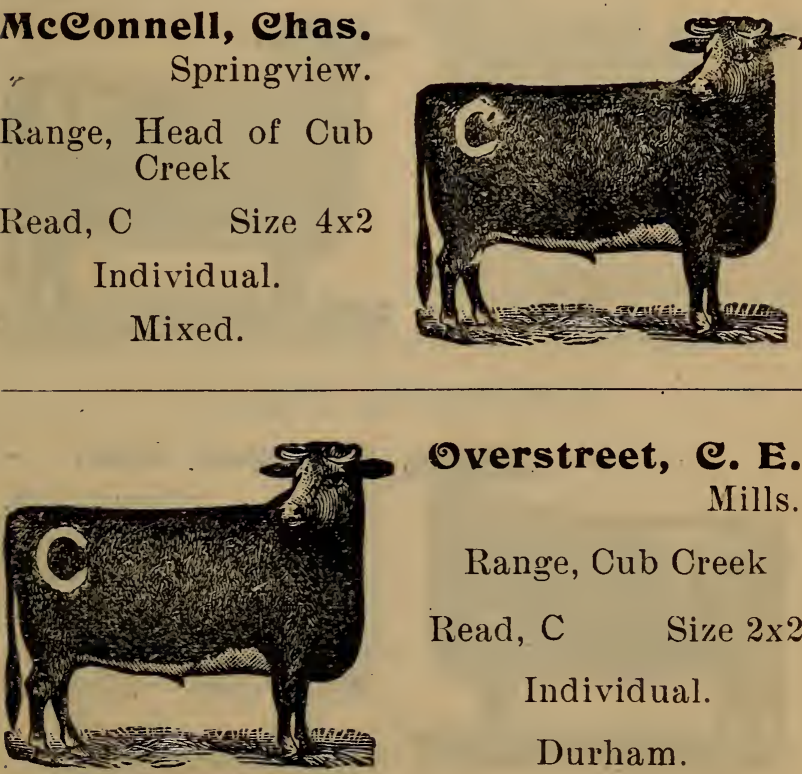

Overstreet, c. E. Mills.

Range, Cub Creek

Read, C Size 2x2

Individual.

Durham. 
Overstreet, T.J.

Mills.

Range, Cub C'reek

Read, C Size 2x?

Individual.

Durham.
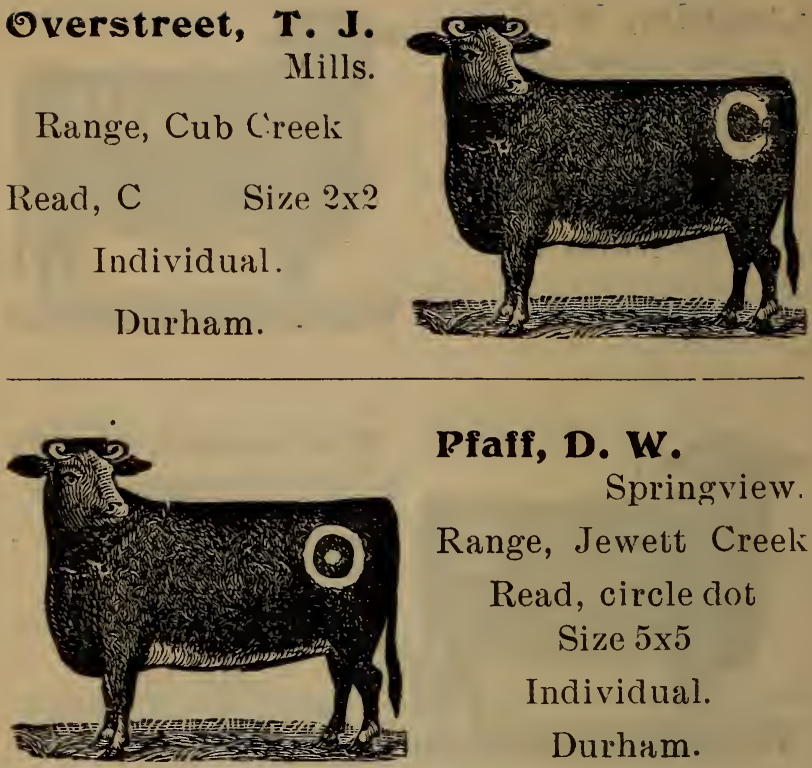

Pfaff, D. W.

Springview.

Range, Jewett Creek

Read, circle dot

Size 5x5

Individual.

Durham.

\section{Smoot, Robt.}

Springview.

Range, Head of Jewett Creek.

Read, 46 Size 5x10 Right Side Individual.

Mixed.
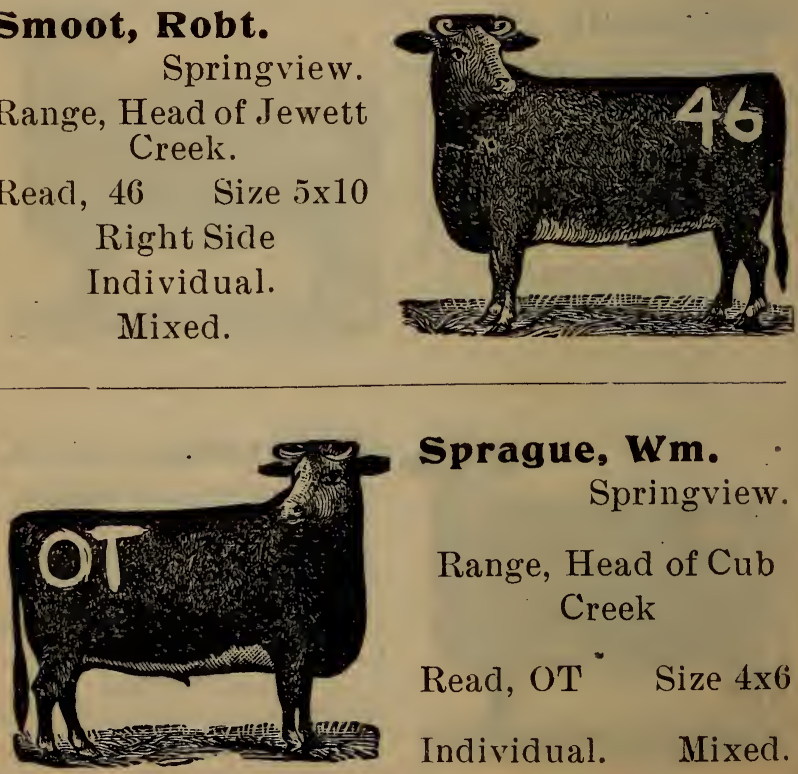
Schrautz, J. S.

Springview,

Range, West Holt Creek

Read, J T Size 4x5 Individual.

Hereford. - Durham.
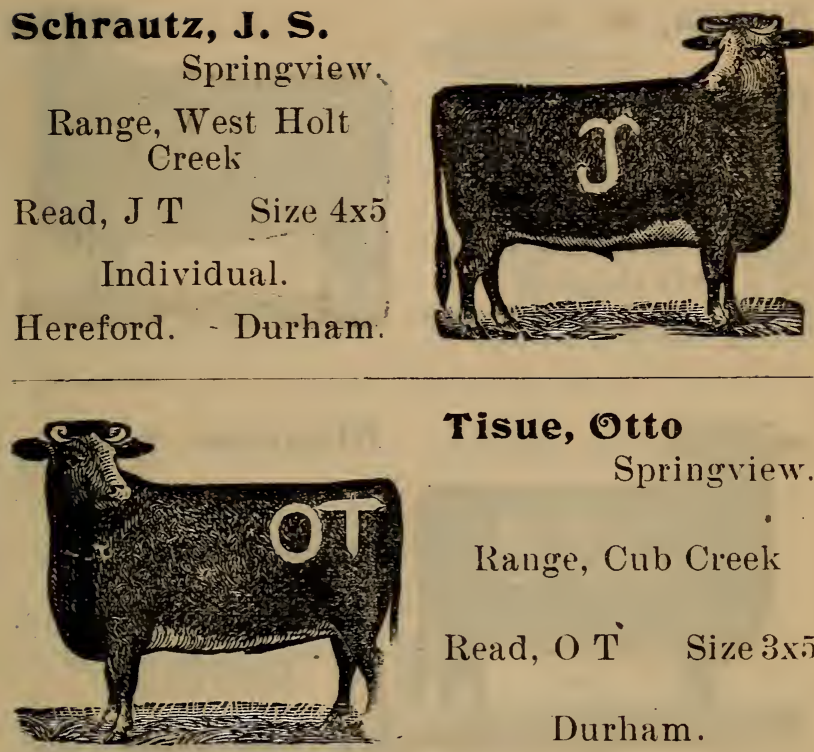

Tisue, Otto

Springview.

Range, Cub Creek

Read, o Ti Size $3 x \overline{5}$

Durham.

\section{Tisue, E. H.}

Springview.

Range, Cub Creek

Read, ET connected Size $4 \mathrm{x} 4$

Individual.

Durham.

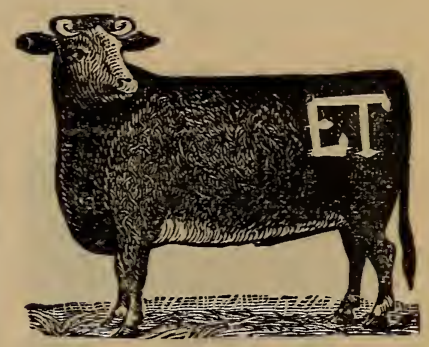

\section{Holt Precinct.}

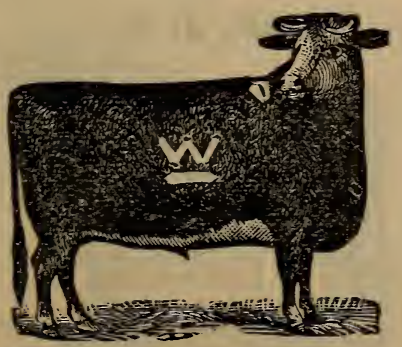

Akers, J. W.

Springview.

Range, Holt Creek

Read, W bar Size $8 \times 8$

on either side

Individual.

Durham. 
Austin, W. A. Springview.

Range, Head of Timber Creek

Read, S open AL Size 6 in.

Horses, inverted $\Omega$ on left thigh.

State. Association.
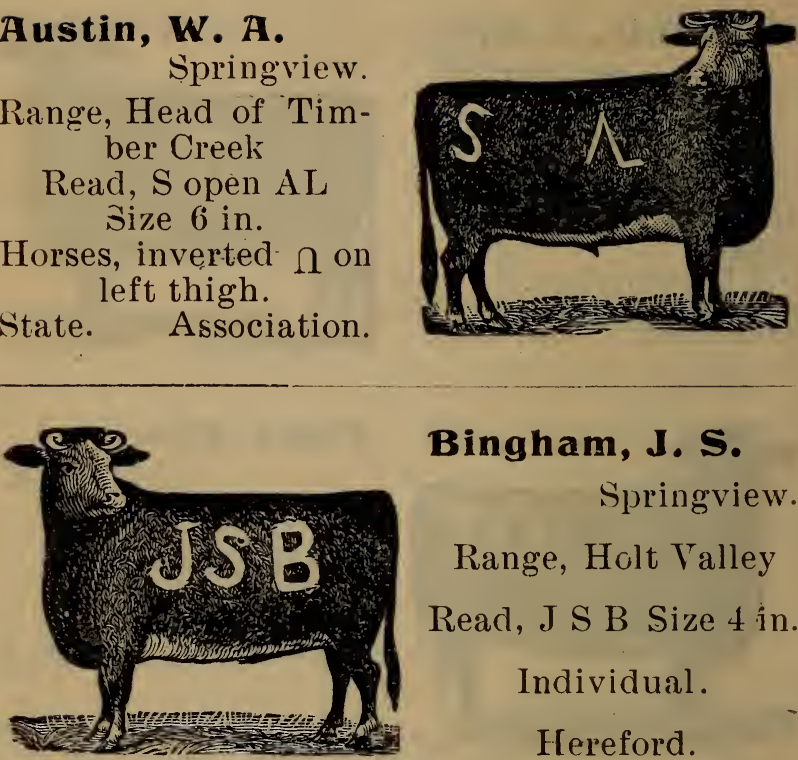

Bingham, J. S.

Springview.

Range, Holt Valley

Read, J S B Size 4 ín.

Individual.

Hereford.

Ballard, Mary Ballard, Geo. E. Springview.

Range, West Holt Read, M Size 5x5

County.

Hereford.
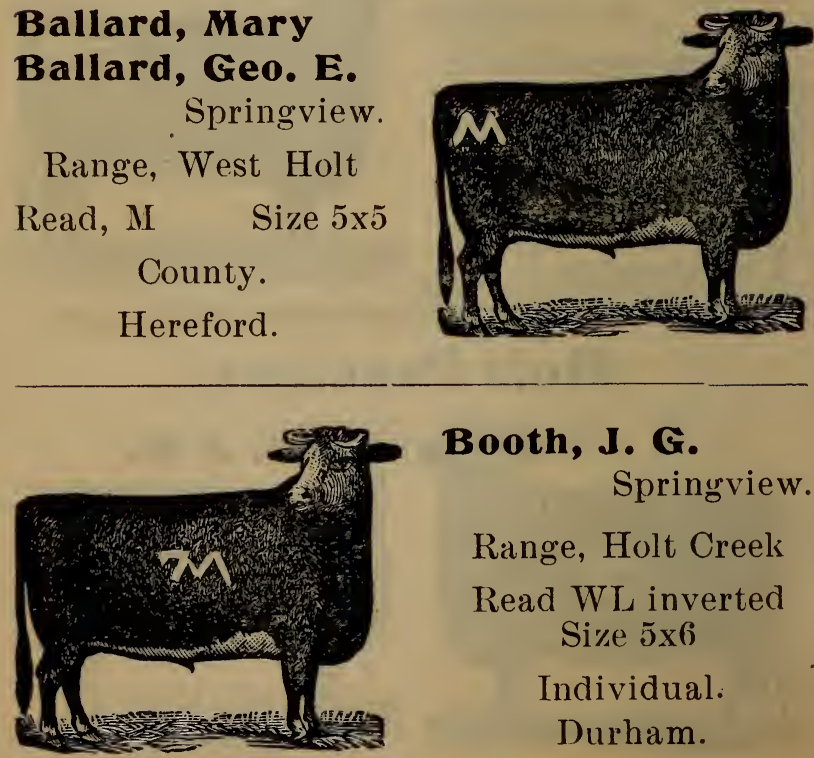

Booth, J. G.

Springview.

Range, Holt Creek

Read WL inverted

Size $5 \times 6$

Individual.

Durham. 
Booth, e. A.

Springview.

Range, Holt Creek

Read, 7 B Size 5x6

Durham.
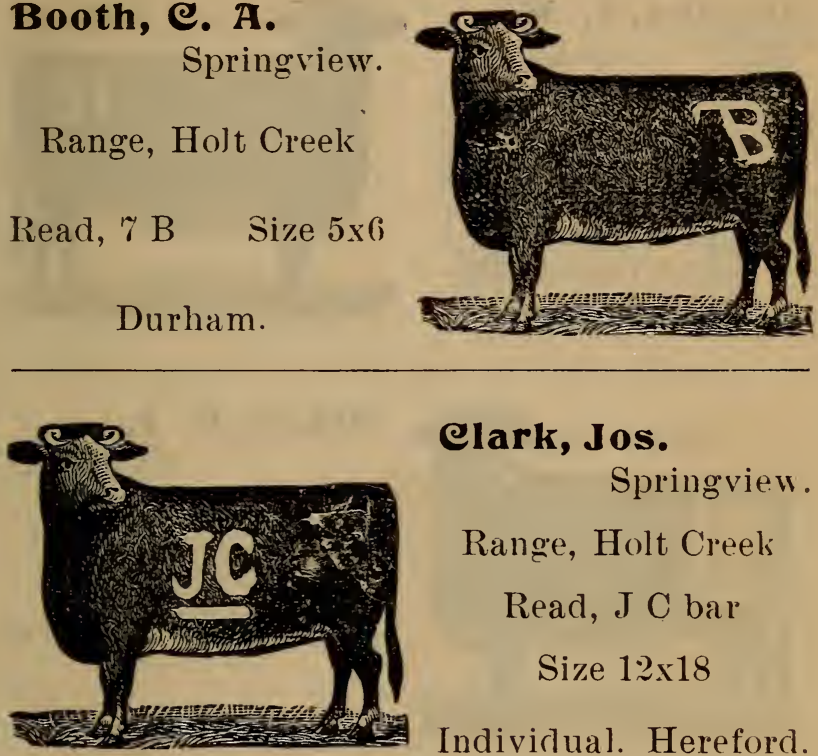

elark, Jos.

Springview.

Range, Holt Creek

Read, J C bar

Size $12 \times 18$

Indivirlual. Hereford.

\section{Carlson, Alfred}

Marlbank.

Range, Cottonwood

Read, A C Size $4 \times 6$ County.

Durham.

Herefords.
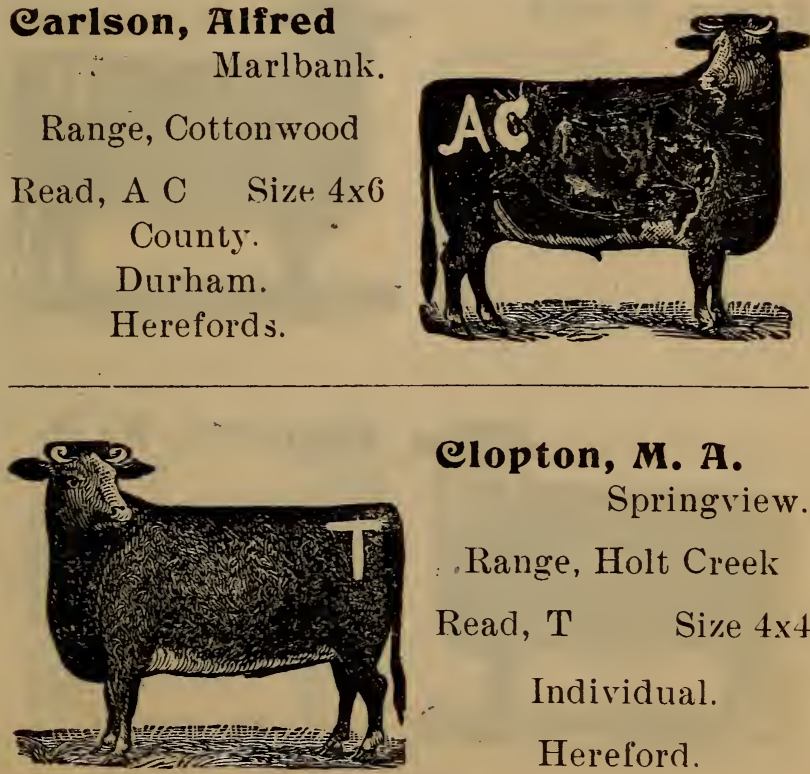

elopton, M. A.

Springview.

.Range, Holt Creek

Read, $T \quad$ Size $4 \times 4$

Individual.

Hereford. 
elopton, I. M.

Springview.

Range, South Cottonwood

Read, I C - Size 3x5

Individual.

Red Polled.

Hereford.
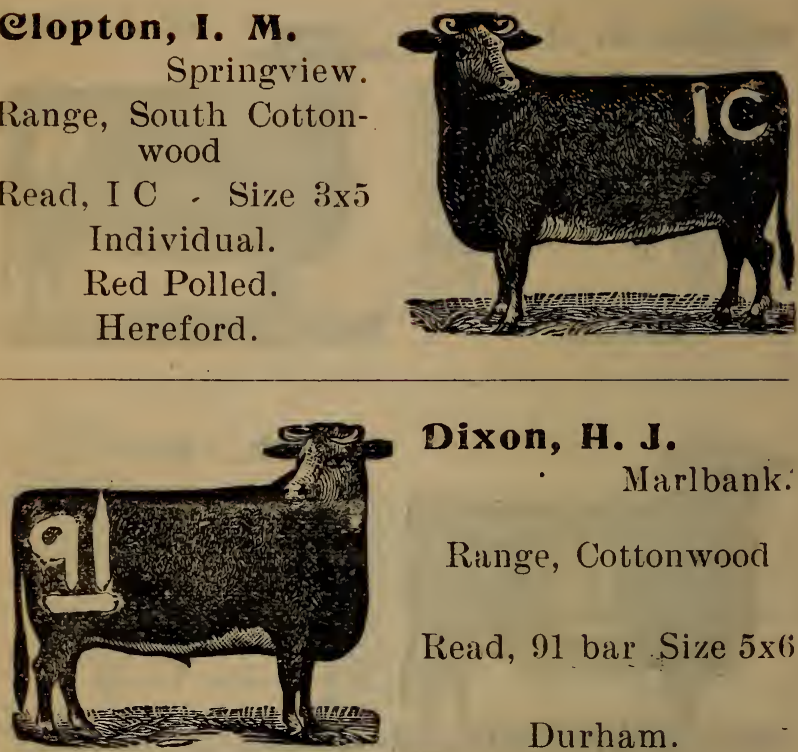

Dixon, H. J.

- Marlbank:

Range, Cottonwood

Read, 91 bar Size $5 \times 6$

Durham.

\section{Dixon, Frank}

Marlbank.

Range, Cottonwood

Read, $91 \quad$ Size 5x

Association.

Durham.
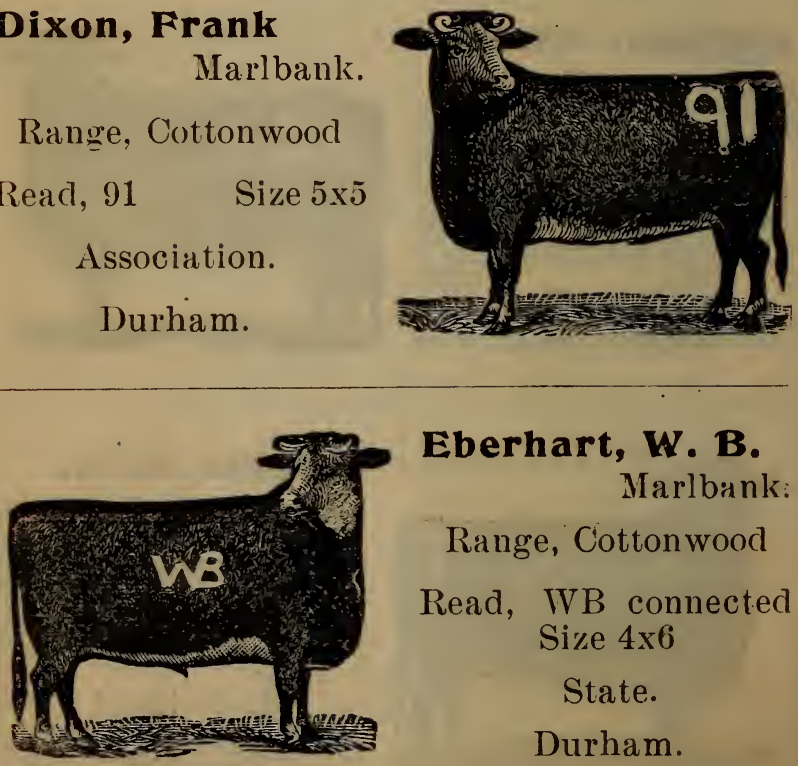

Eberhart, W. B.

Marlbank:

Range, Cottonwood

Read, IVB connected Size $4 \times 6$

State.

Durham. 


\section{Fink Bros.}

Springriew.

Range, Head of Cottonwood

Read, $X 7$ Size $4 \times 5$

State.

Hereford.

Short Horn.
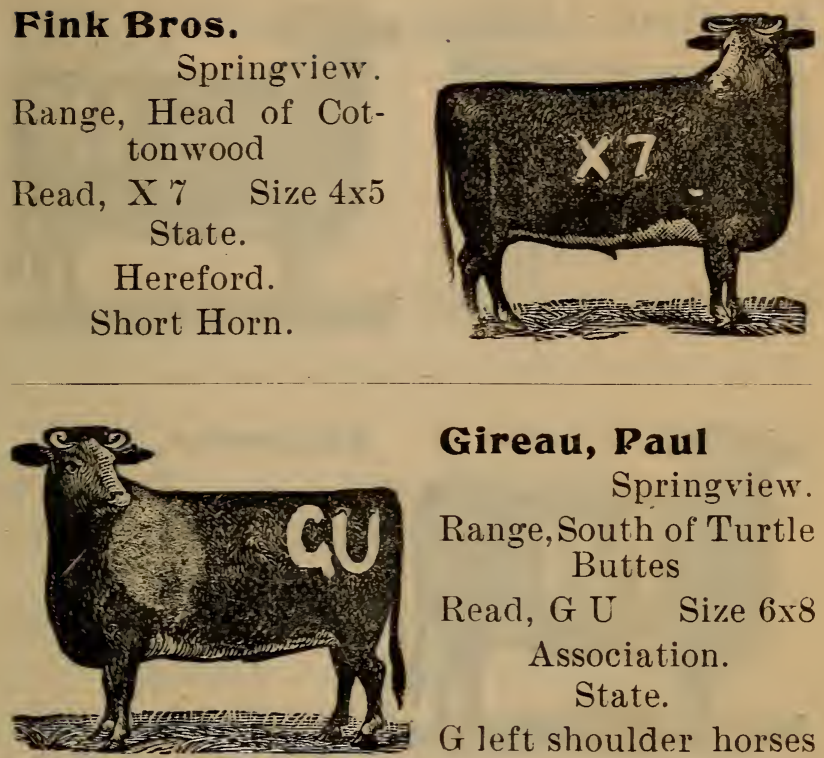

Gireau, Paul

Springview.

Range, South of Turtle Buttes

Read, G U Size 6x8 Association.

State.

G left shoulder horses

\section{Huston, Eilert}

Norden.

Range; between Cottonwood \& Lost Creeks Read, 110 Size 3 in.

County.

Mixed.
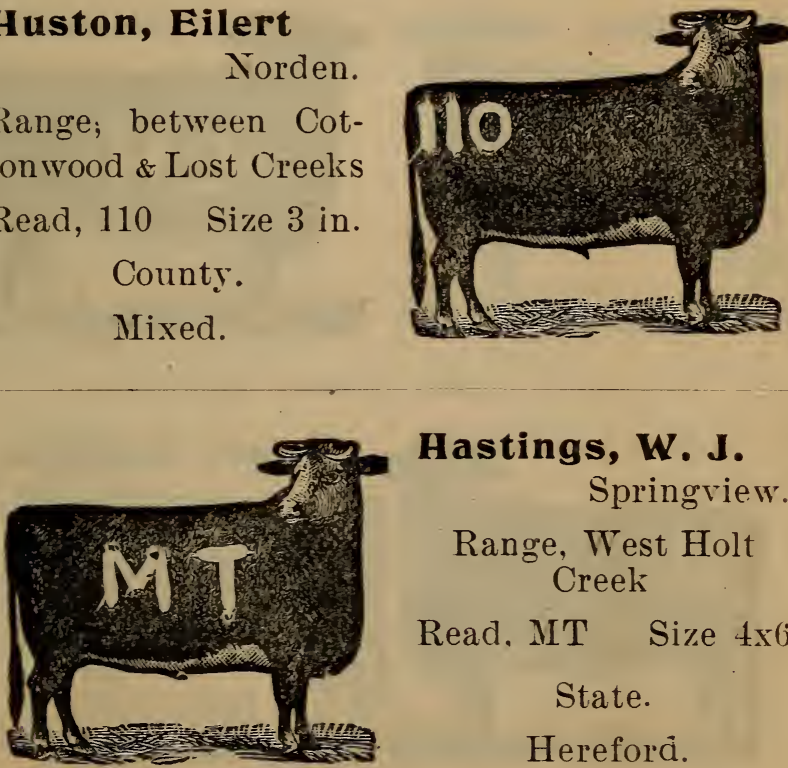

Hastings, W. J.

Springview.

Range, West Holt Creek

Read, MT Size $4 \times 6$.

State.

Hereford. 
Hamilton, L. L.

Springview.

Range, Holt Creek

Read, L H Size $4 \times 5$

Individual

Durham.
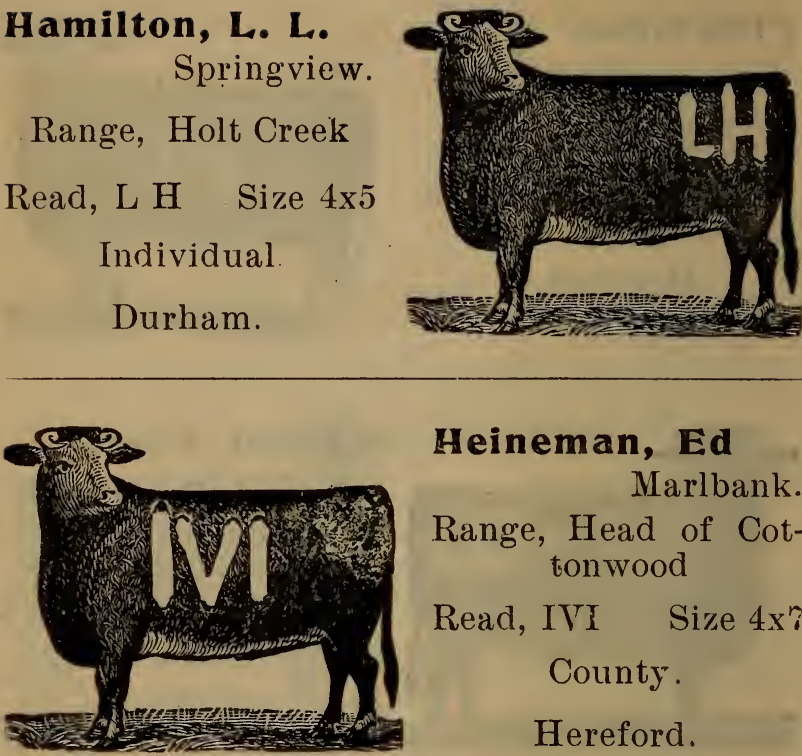

Heineman, Ed

Marlbank.

Range, Head of Cottonwood

Read, IVI Size 4x?

County.

Hereford.

\section{Hamilton, e. A. Springview.}

Range, Holt Creek

Read, L H Size $4 \times 5$

Durham.
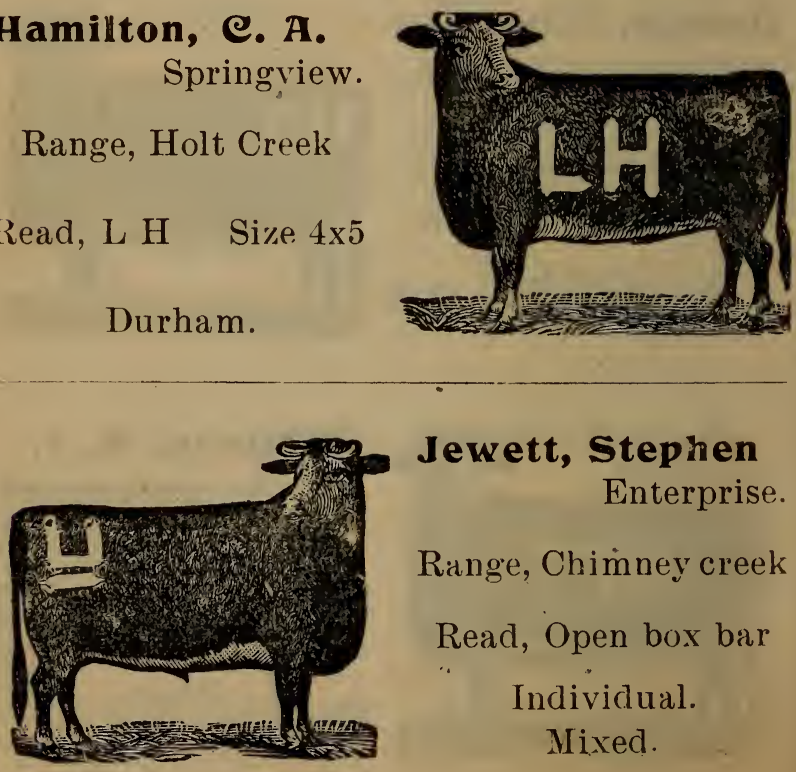

Jewett, Stephen

Enterprise.

Range, Chimney creek

Read, Open box bar

Individual.

Nixed. 


\section{5.)}

Kurzenberger, William

Springview.

Range, Holt Creek

Read, Scissors

Size $6 x 5$

County.

Durham.
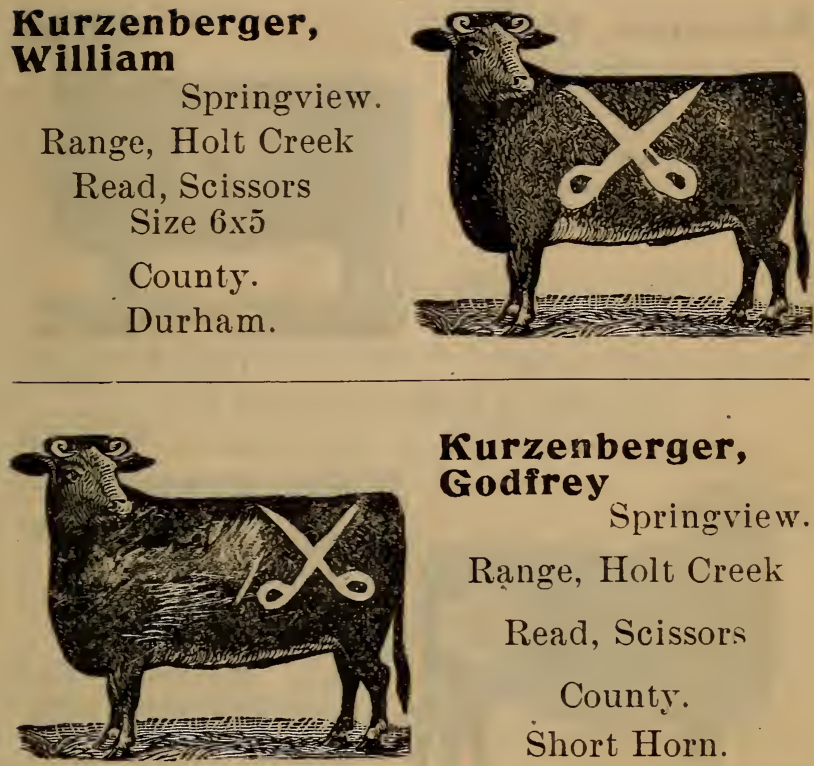

Kurzenberger, Godfrey

Springview.

Range, Holt Creek

Read, Scissors

County.

Short Horn.

\section{Kuhl, Henry}

Springview.

Range, West Holt

Read, Half Leg Size 7 in.

Individual.

Shơt Horn.
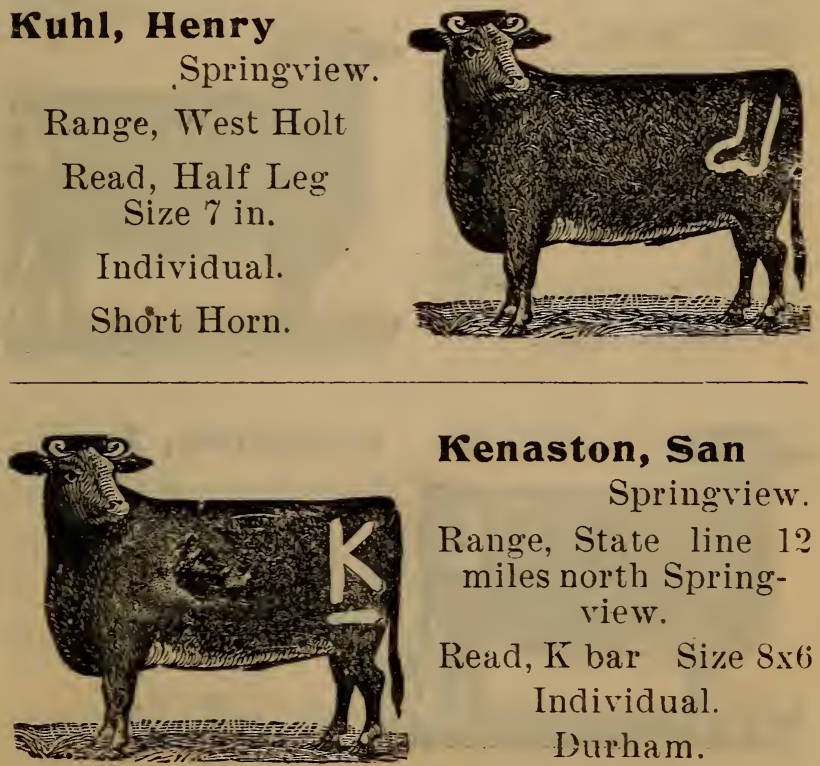

Kenaston, San

Springrview.

Range, State line 1? miles north Springview.

Read, $K$ bar Size $8 \times 6$ Individual. Durham. 
Kenaston, W. A.

Springview.

Range, State line 12 miles north Springview

Read, Two-thirds Size $12 \times 8$.

Association.

Dulap mark on all.

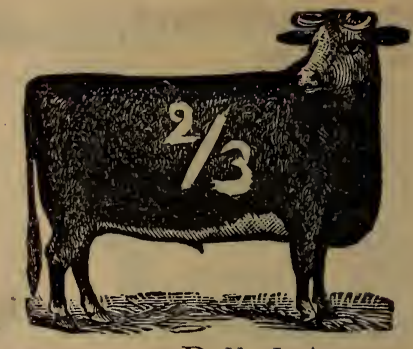

Old brand $\mathrm{K}$ bar on right hip.

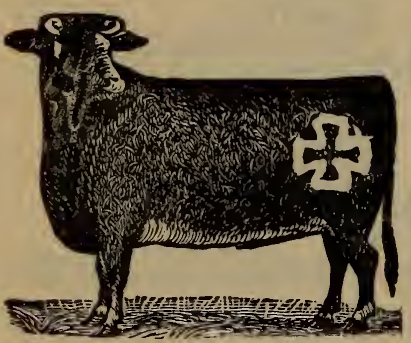

\section{Lear, e. E.}

Springview.

Range, Holt Creek

Read, Maltese Cross Size $6 \times 6$

Association. Durham.

\section{Lear, $\mathrm{Wm}$.}

Springview.

Range, Holt Creek

Read, WL connected on Left Side

Size $5 \times 6$.

Ass'n.

Short Horn.

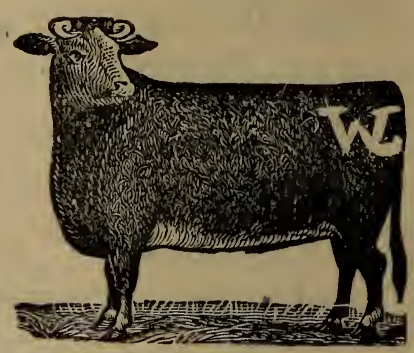

Loughran, T. F.

Marlbank.

Range, Cottonwood Valley

Read, OL Size 5x'7

County.

Mixed. 


\section{7}

Mock, John

Marlbank.

Range, 4 miles north Norden.

Read, I Y Bar Size $5 x$ '

Mixed.

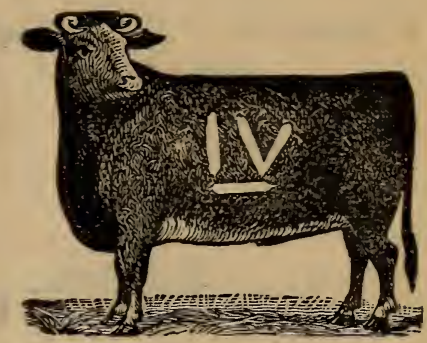

Mock, Mrs. F.

Marlbank.

Range, Cottonwood

Read, I V Size 5x'

Mixed.

\section{Neihus, Claus}

- Marlbank.

Range, Cottionwood

Read, C N Size 5x?

State.

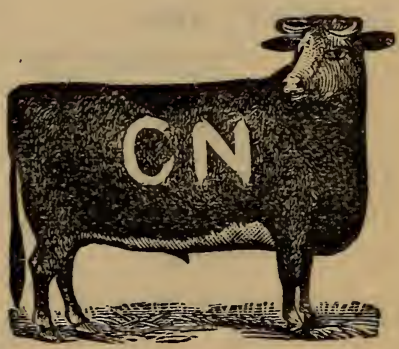

Niehus, Frank Springview.

Range, West Holt Creek

Read, $5 \mathrm{~N} \quad$ Size $4 \mathrm{x} 4$

State.

Durham.

Ass'n. 
Pense, A.

Springview.

Range, West Holt Creek

Read, PW

Size $3 \frac{1}{2}$ in. each

County. Hereford. Durham.
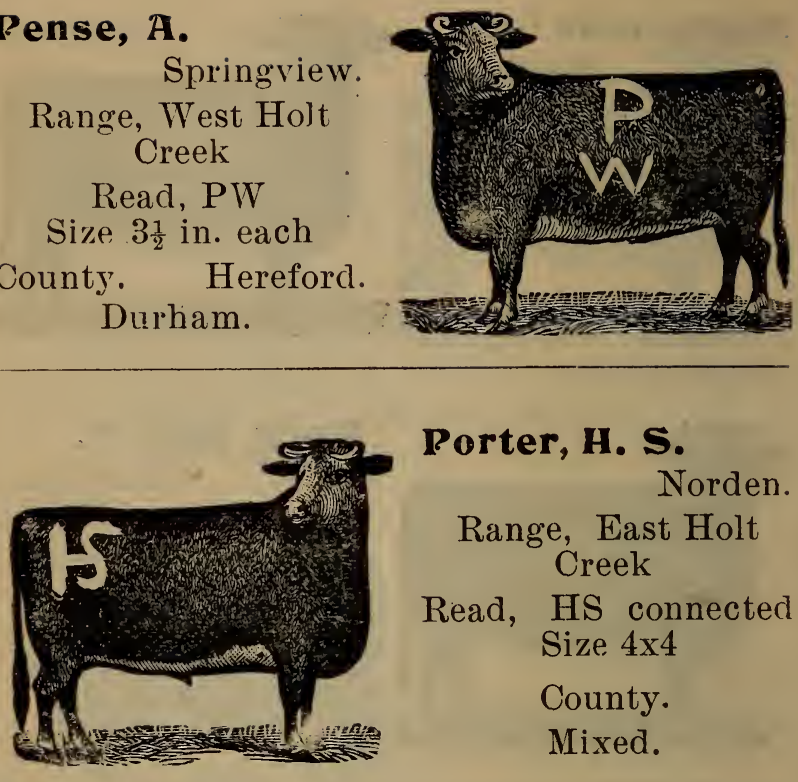

\section{Ev uther, Jos.}

Springview.

Range, West Holt Read, J R Size $4 \times 3$ County. Red Polled.

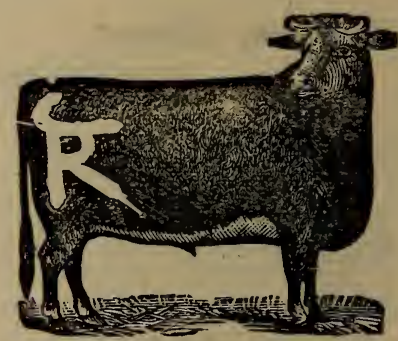

\section{Roby, D. K.}

Springview.

Range, Cottonwood Creek

$$
\text { Read, D bar R }
$$

Size $5 \times 17$

Ass'n.

Durham. 
Snyder, E. L.

Springview .

Range, West Holt Read, 5 S Size $5 \times 10$ State.

Durham.

Horse brand $\mathbf{Z Z}$ on left thigh

Read, double $\mathbf{7}<$
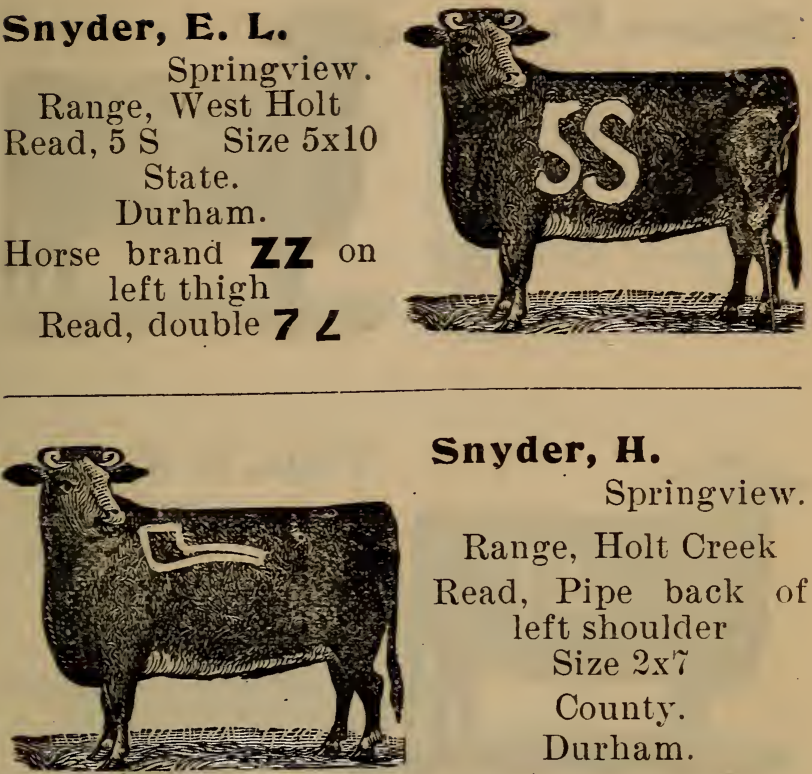

Snyder, H.

Springview.

Range, Holt Creek

Read, Pipe back of left shoulder

Size $2 x \%$

County.

Durham.

\section{Shattuck, L. H.}

Marlbank.

Range, Cottonwood

Read, S Size $3 \times 4$

Individual.

Mixed.
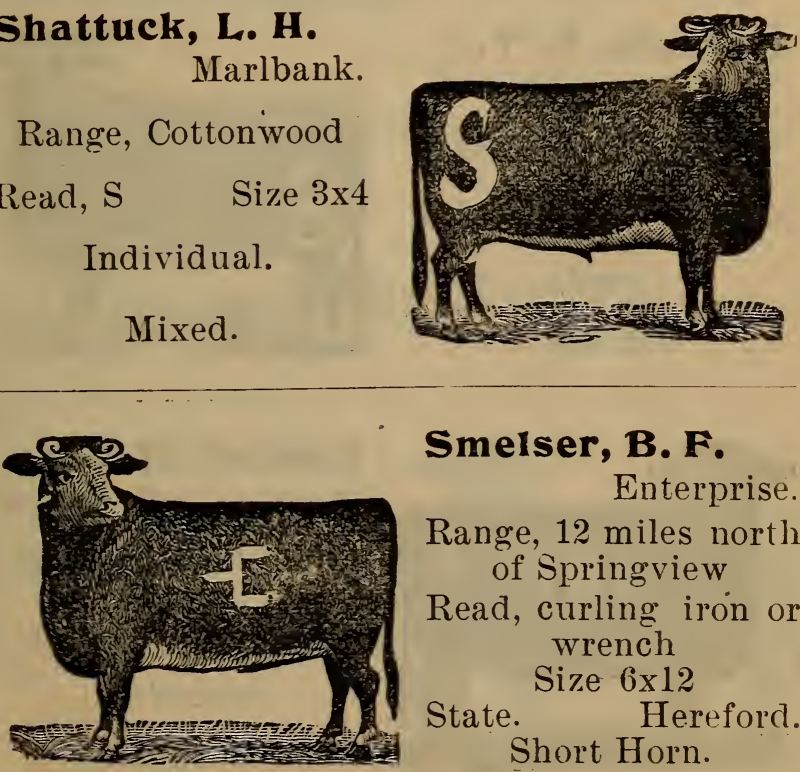

\section{Smelser, B. F.}

Enterprise.

Range, 12 miles north of Springview

Read, curling iron or wrench

Size $6 \times 12$

State. Hereford. Short Horn. 
60

Thomas, W.F.

Springview.

Range, West Holt Creek.

Read, lazy H T Size 10x5

Short Horns.
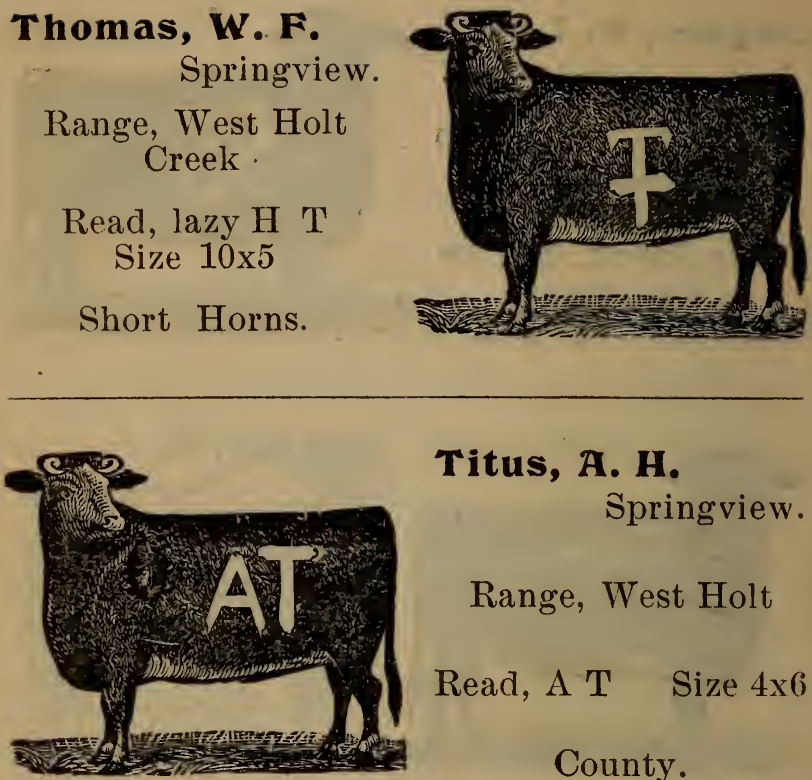

Titus, A. H.

Springview.

Range, West Holt

Read, A T Size $4 \times 6$

County.

Vifquain, E. $T$.

Springview.

Range, West Holt

Read, 8

Size $4 \mathrm{x} 4$

County.

Durham.
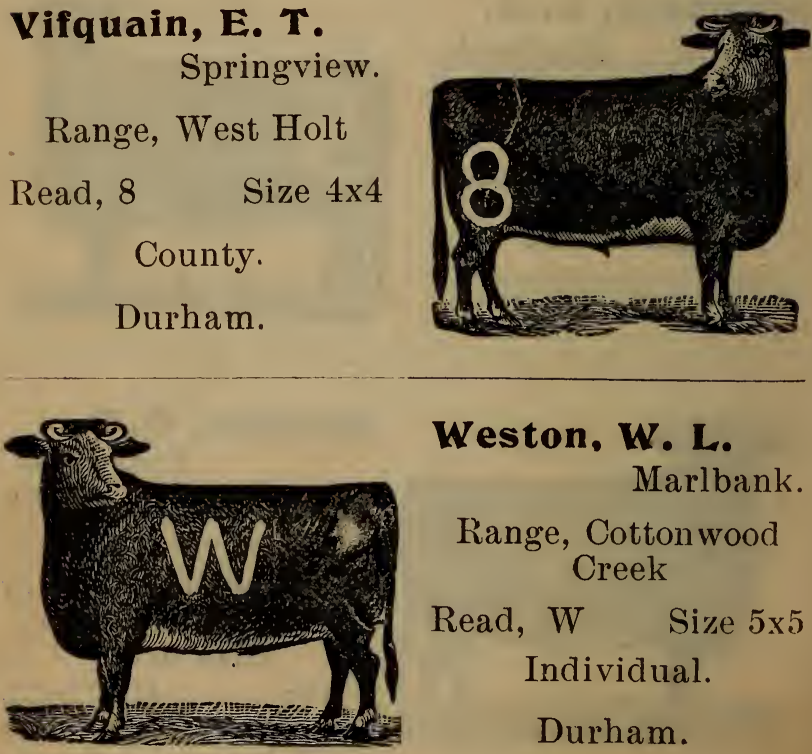

Weston, W. L.

Marlbank.

Range, Cottonwood Creek

Read, W Size 5x5

Individual.

Durham. 
Woeppel, Gus Springview.

Range, Timber Creek

Read, G U S bar

Size 6 in. each

State.

Hereford.
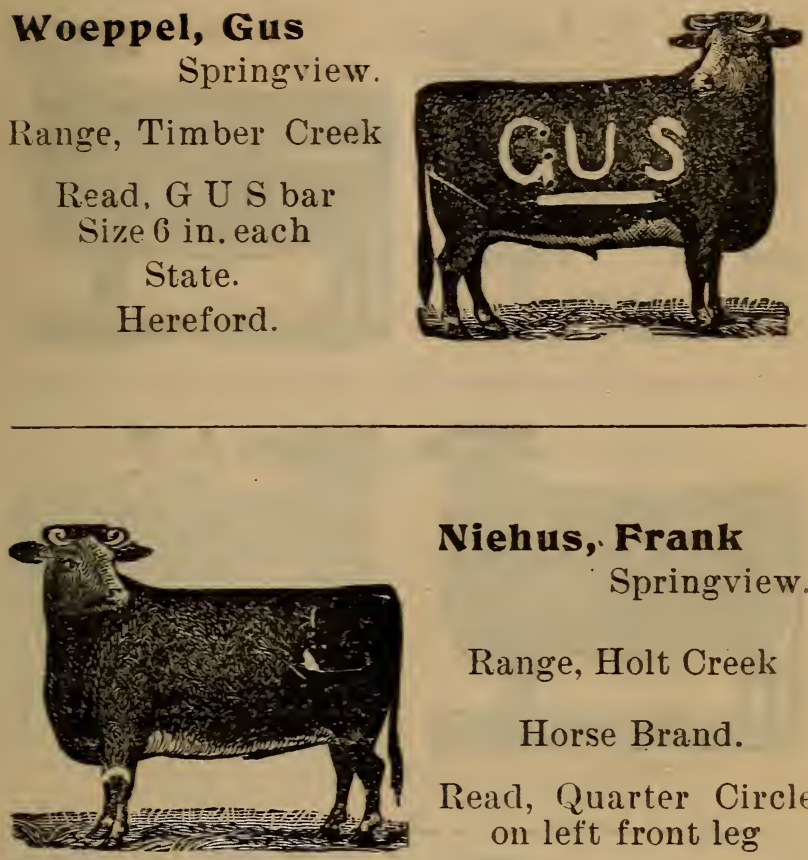

Niehus, Frank

Springview.

Range, Holt Creek

Horse Brand.

Read, Quarter Circle on left front leg

\section{Keya Paha Precinct.}

Adkins, R. M.

Brocksburg.

Range, Spotted Tail Creek

Read, A $4 \quad$ Size 5x6

State.

Mixed.

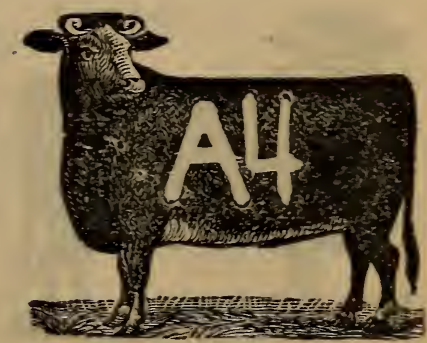


Baker, A. J.

Brocksburg.

Range, Northeast of Mills

Read, carpenter's

Size $3 \times 5$. County. Mixed.

Horses side of neck
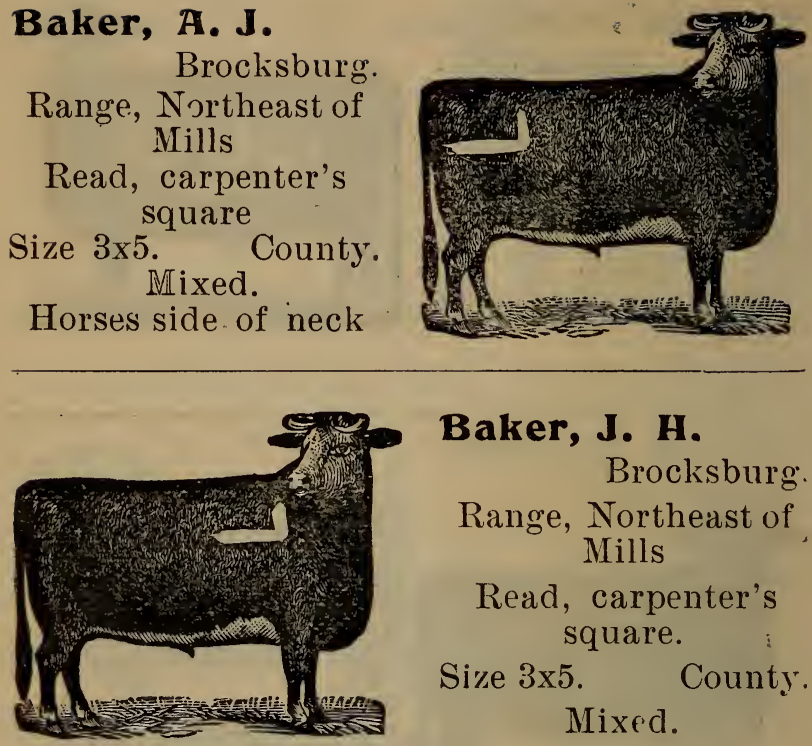

\section{Baker, J. H.}

Brocksburg.

Range, Northeast of Mills

Read, carpenter's square.

Size $3 \times 5$.

County.

Mixed.

Horse same on left side of reck.

\section{Bauld, John}

Brocksburg.

Range, Coon Creek

Read B5 Size 5x6

Ass'n. - State.

Hereford.

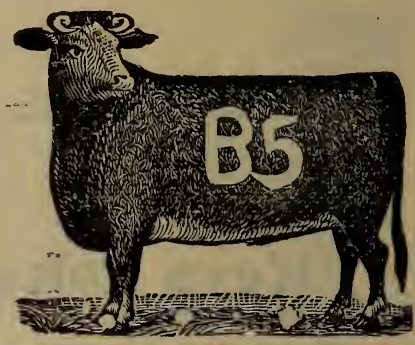

\section{Briscoe, Jos.}

Brocksburg.

Range. betw'n Brocksburg and Ponca

Read, J B connected County. Association. 
Collins, W. M.

Brocksburg.

Range, Wolf C'reek

Read, YC

Hereford.

Old brand pad lock
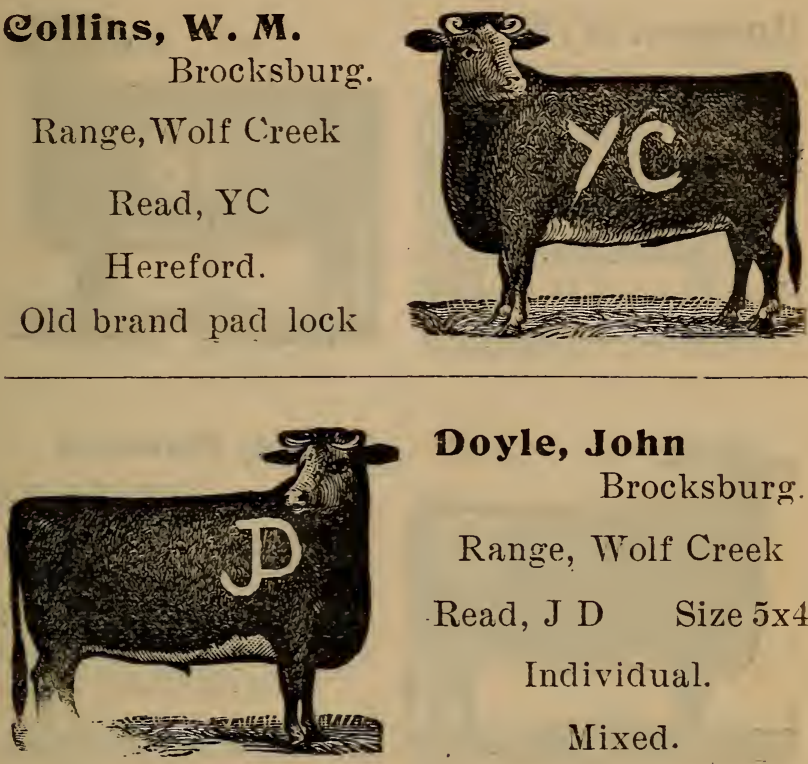

Doyle, John

Brocksburg.

Range, Wolf Creek

Read, J D Size 5x4

Individual.

Mixed.

Ditto, elark N.

Brocksburg.

Range, Keya Paha River

Read, C L Size $4 \times 8$

Individual.

Short Horn. Hereford.
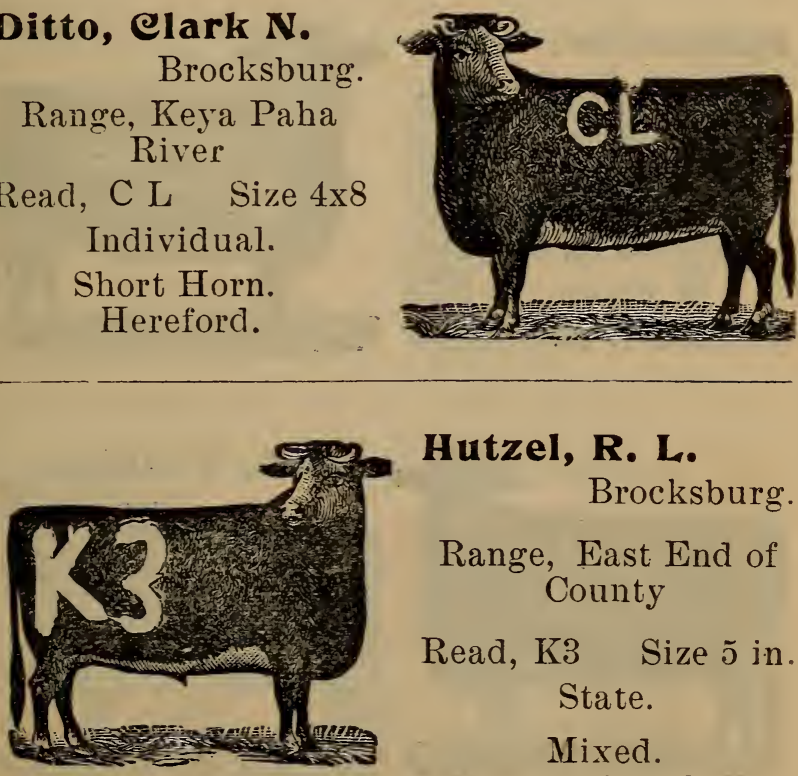

\section{Hutzel, R. L.}

Brocksburg.

Range, East End of County

Read, K3 Size 5̃ in. State.

Mixed. 
Hudson, W. N.

Brocksburg.

Range, Oak Creek Read, S 8 Size $6 \times \mathbf{r}$ State. Ass'n.

Hereford.

Horse brand $\mathrm{H}$ bar on left shoulder
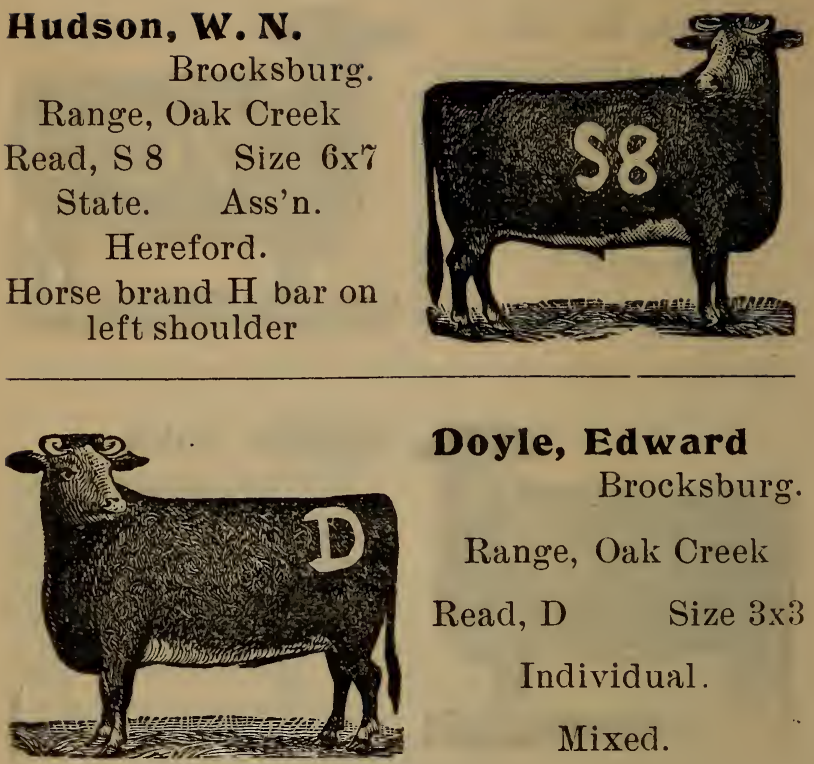

Doyle, Edward

Brocksburg.

Range, Oak Creek

Read, D Size $3 \times 3$

Individual.

Mixed.

Fickweiler, Willy

Brocksburg.

Range, Keya Paha river

Read, Six Shooter

Size 13 in. long

State.

Mixed.
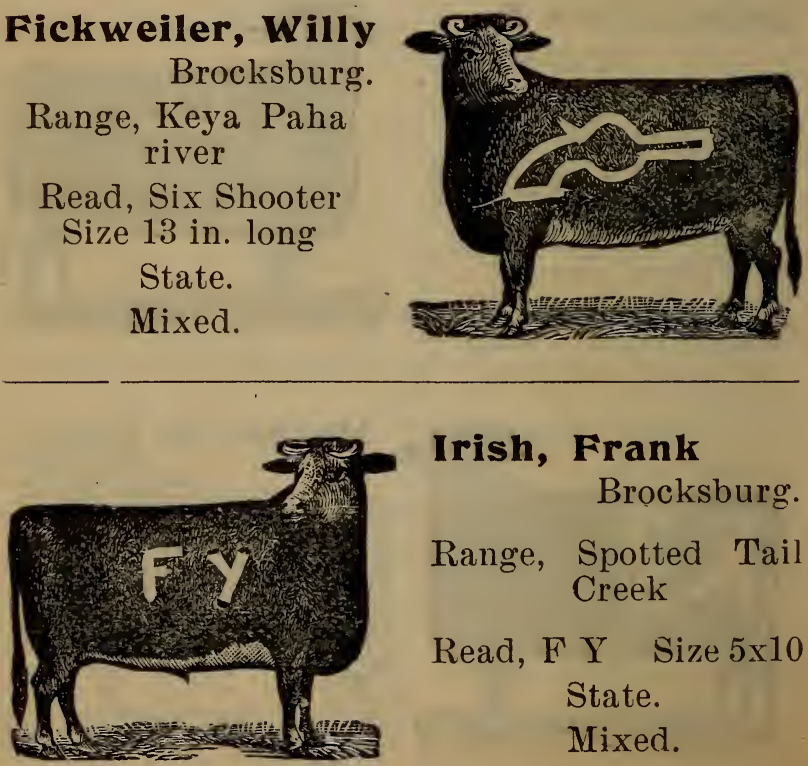

Irish, Frank

Brocksburg.

Range, Spotted Tail Creek

Read, F Y Size 5x10 State.

Mixed. 
Jeffords, Mary A.

Brocksburg.

Rangie, Wolf Creek

Read, Anchor

Individual.

Short Horn.
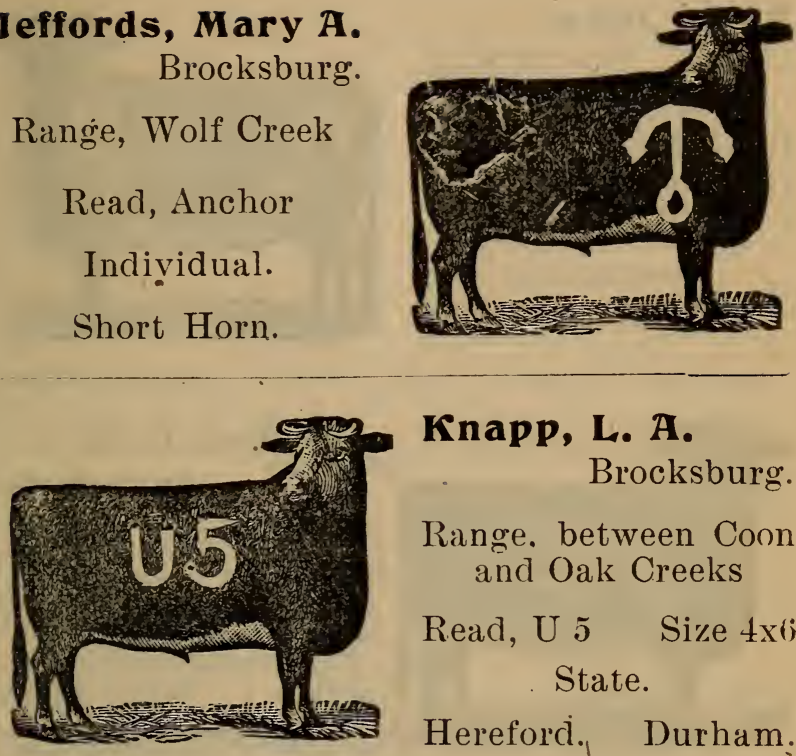

Knapp, L. A.

Brocksburg.

Range. between Coon and Oak Creeks

Read, U $5 \quad$ Size $4 \times 6$

State.

Hereford. Durham.

Lusk, W. K.

Brocksburg.

Read, $3 \mathrm{X}$ bar

Size $4 x 4$

County.

Mixed.

Same brand for horses

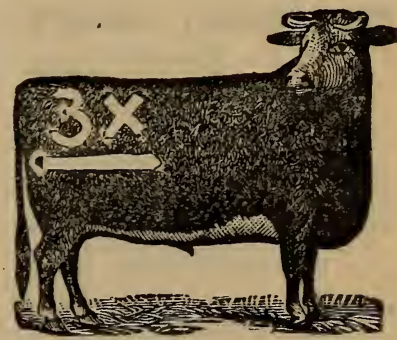

\section{Munt, Frank}

Brocksburg.

Range, Oak Creek

Read, C C reversed Size $4 \times 4$

Individual.

Mixed. 


\section{Payn, John}

Brocksburg.

Range, Wolf Creek Read, P A Șize 5 in. $\mathrm{P}$ on Left Shoulder, A on Left Thigh Association. Mixed.
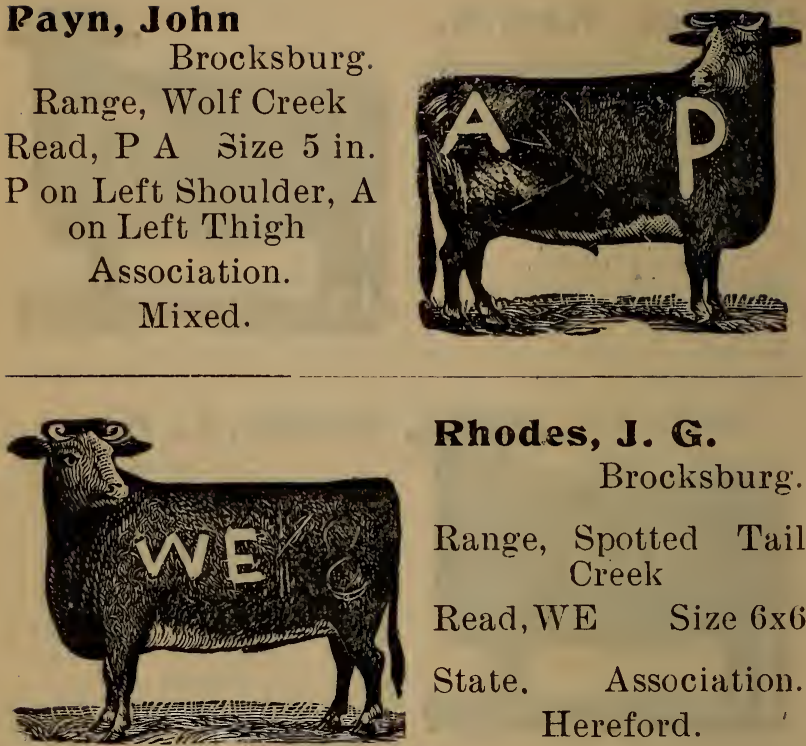

\section{Rhodes, J. G.}

Brocksburg.

Range, Spotted Tail Creek

Read,WE Size $6 \times 6$

State. Association. Hereford.

\section{Swetland, Henry}

Brocksburg.

Range, Keya Paha river

Read, V S Size 5x6 Horses on left shoulder Association.

State. Hereford.

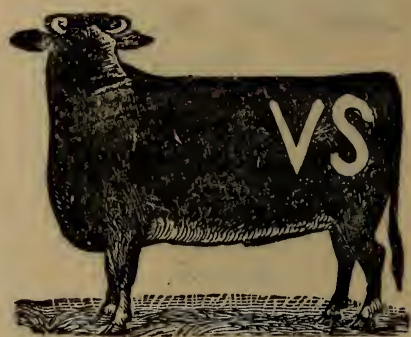
Old brand HS connected.

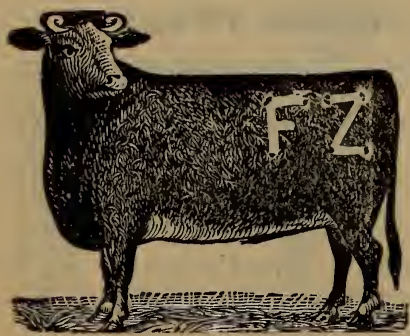

Satterlee, F. E. and $G$. $Z$.

Brocksburg.

Range, Keya Paha river near Brocksburg Read FZ Size 4 in. State. Durham. Polled Angus. 
Stewart, Jack

Mills.

Range, Wolf Creek

Read, S on forehead

Size $4 \frac{1}{2} \times 3$

Individual.
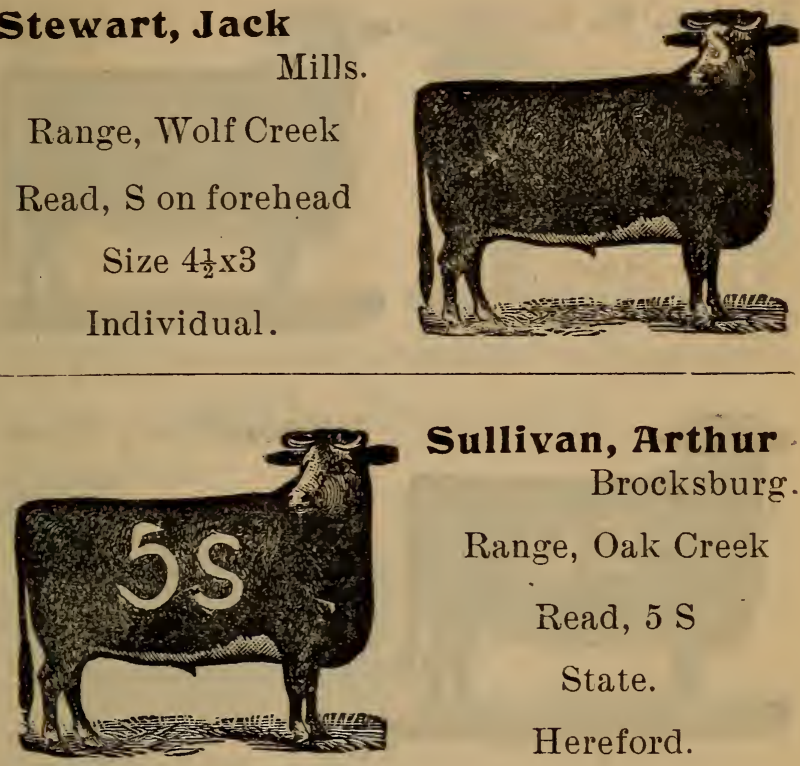

\section{Sullivan, Arthur}

Brocksburg.

Range, Oak Creek

Read, $5 \mathrm{~S}$

State.

Hereford.

\section{Thompson, John}

Brocksburg.

Range, Keya Paya river

Read, J T bar

Size $4 \mathrm{x} 6$

County.

Mixed.
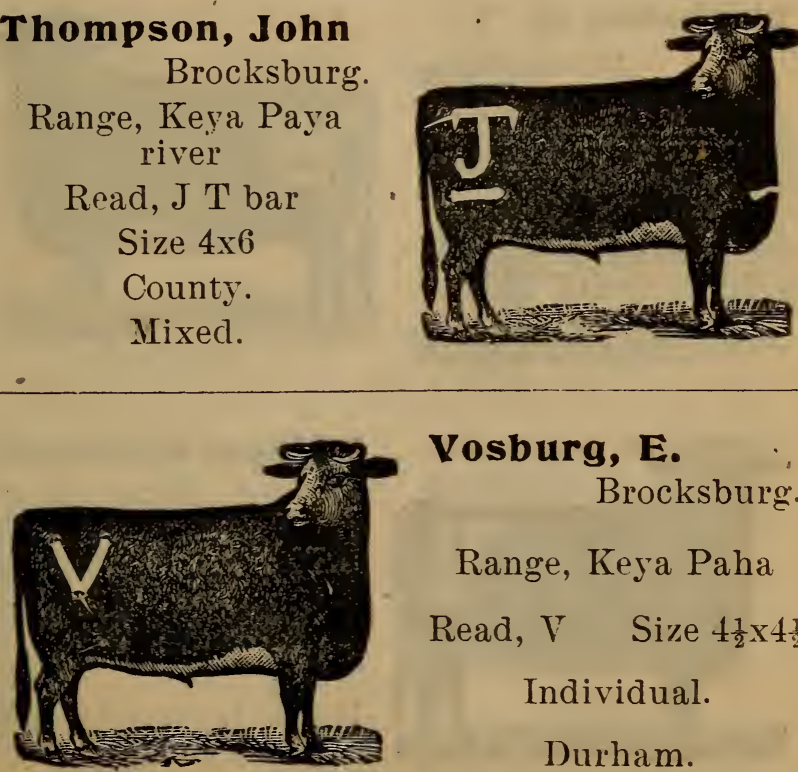

Vosburg, E.

Brocksburg.

Range, Keya Paha

Read, V Size $4 \frac{1}{2} \times 4 \frac{1}{2}$

Individual.

Durham. 
Wales, H. E.

Naper.

Range, Northeast Keya Paha County Read, Necktie and Breeching Size 9 in. State.

Durham.
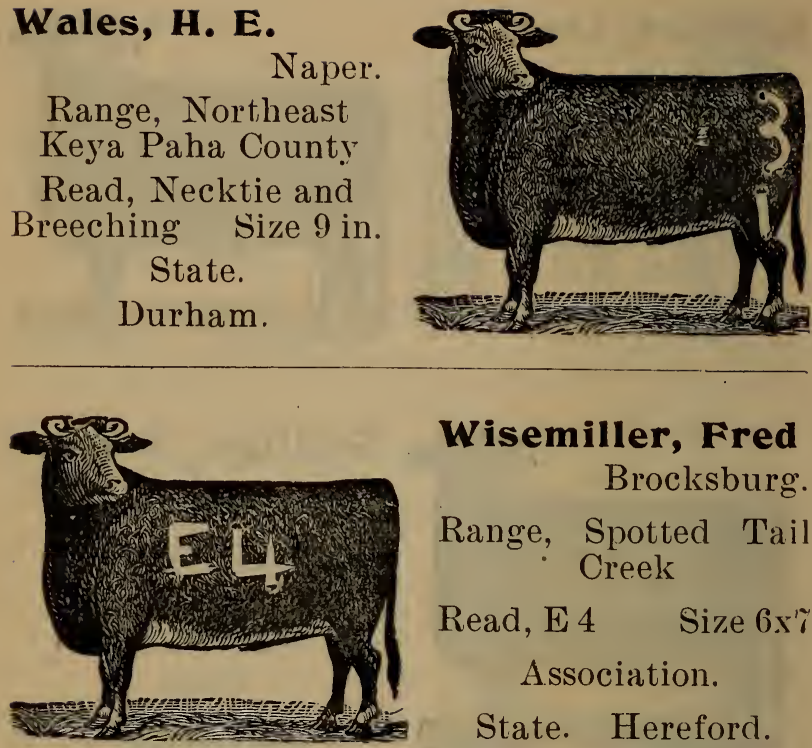

Wisemiller, Fred

Brocksburg.

Range, Spotted Tail Creek

Read, E 4 Size $6 \mathbf{x}^{\prime}$ Association.

State. Hereford.

Williams, M. T.

Brocksburg.

Range, Brocksburg

Read, Seven W Bar Size $6 \times 10$

Association. State.

Horse brand $\mathrm{X}$ on left shoulder.
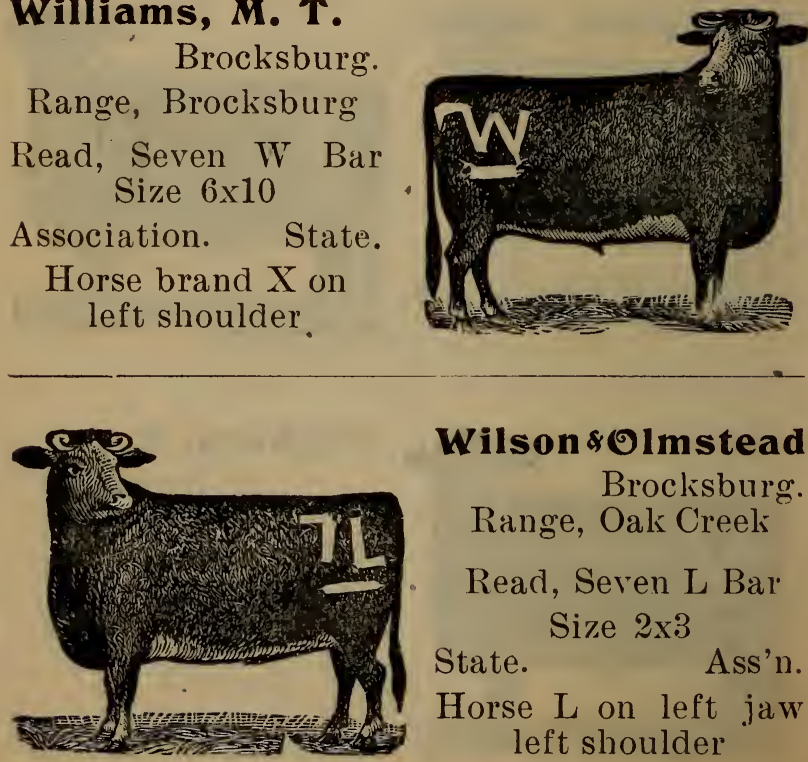

Wilson $\$ O O M s t e a d$

Brocksburg. Range, Oak Creek

Read, Seren L Bar

Size $2 \times 3$

State. Ass'n.

Horse $L$ on left jaw left shoulder 
Wales, E. E.

Naper.

Range, Spotted Tail Creek

Read, E Size 7 in. County.

Durham. White Faces.

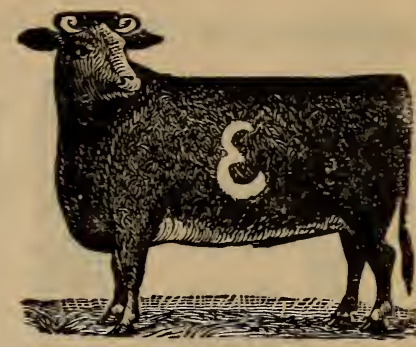

\section{Mills Precinct.}

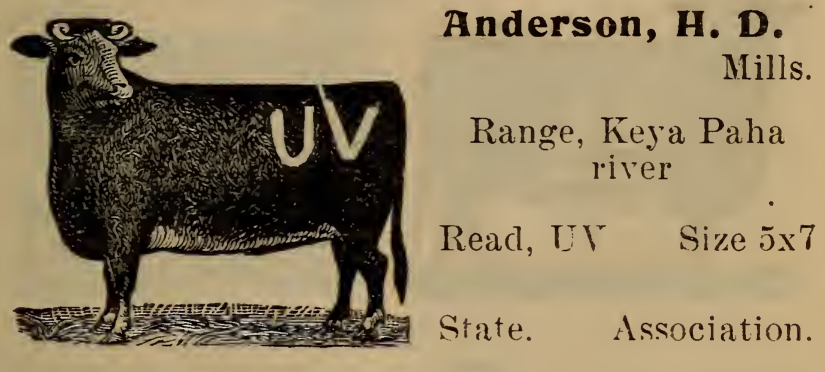

\section{Billings, Alonzo}

Mills.

Range, Pasture

Read, Z B bar

'state. Hereford.

Durham.
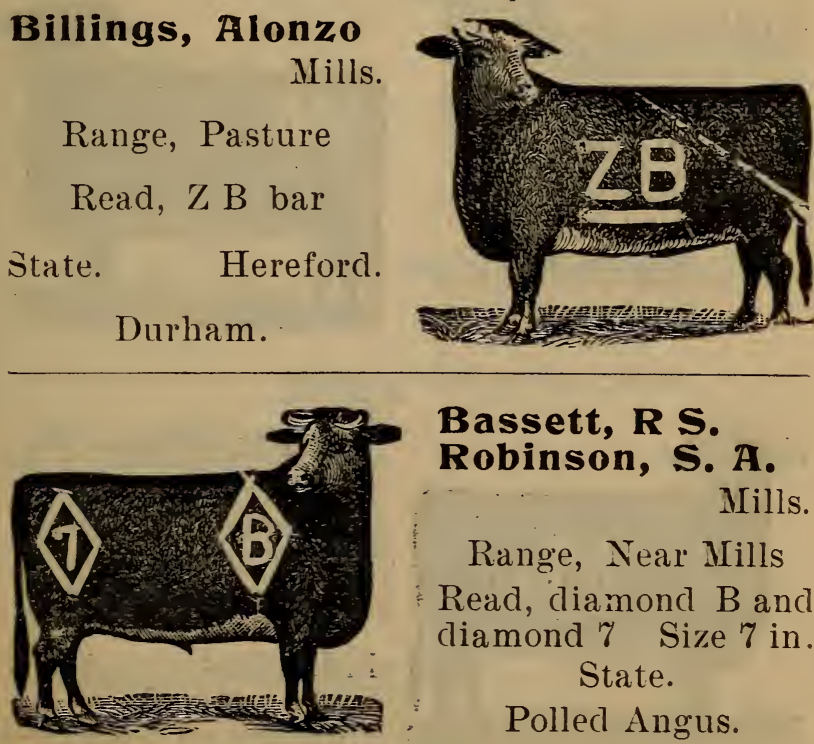

Bassett, R S. Robinson, S. A. Mills.

Range, Near Mills Read, diamond B and diamond 7 Size 7 in. State. Polled Angus. 
Chapin, W. A.

Mills.

Range, Cuon Creek

Read, U C right side Size $5 \times 5$

State.

Durham.
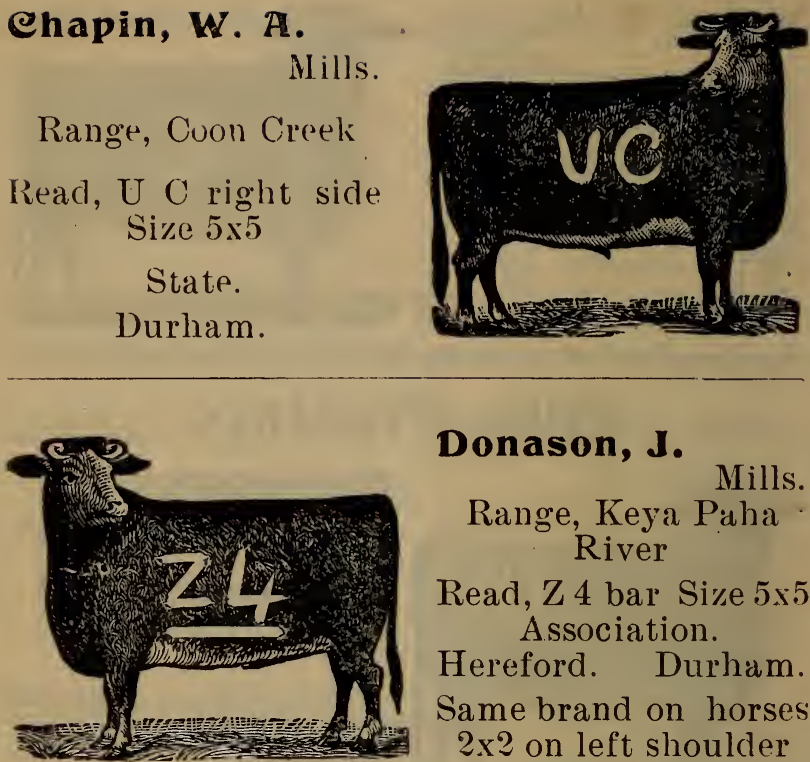

Donason, J.

Mills.

Range, Keya Paha River

Read, Z 4 bar Size 5x5 Association.

Hereford. Durham. Same brand on horses $2 \times 2$ on left shoulder

\section{Knight, W. M.}

Mills.

Range, $1 \frac{1}{2}$ miles west of Mills

Read, WK connected Size $3 \times 5$

Hereford.

Durham.
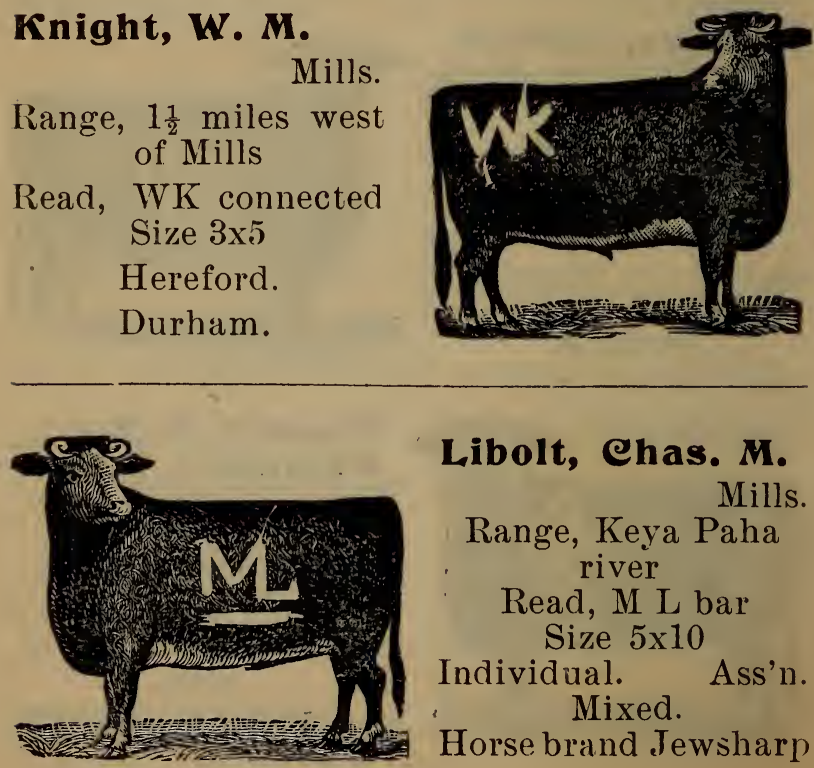

Libolt, ehas. $M$.

Mills.

Range, Keya Paha river

Read, M L bar

Size $5 \times 10$

Individual. Ass'n. Mixed.

Horse brand Jewsharp 


\section{Pratt, M. E.}

Mills.

Range, Indian Creek Read, "Hat brand"

on both hips State.

Association.

Horses \& mules same brand near shoulder.
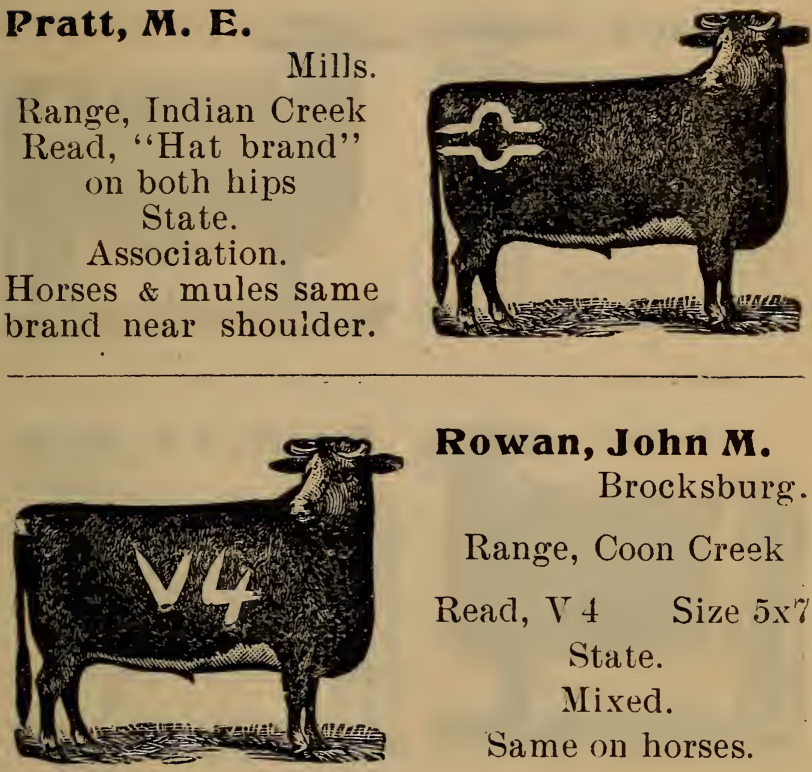

Rowan, John M. Brocksburg.

Range, Coon Creek

Read, V 4 Size $5 x \%$

State.

Mixed.

Same on horses.

\section{Rowan, Thos.}

Mills.

Range, N. E. Keya Paya county

Read, Flying $\mathrm{V}$ and $3 \mathrm{~V}$ Size $6 \times 7$ Association. State. Mixed.
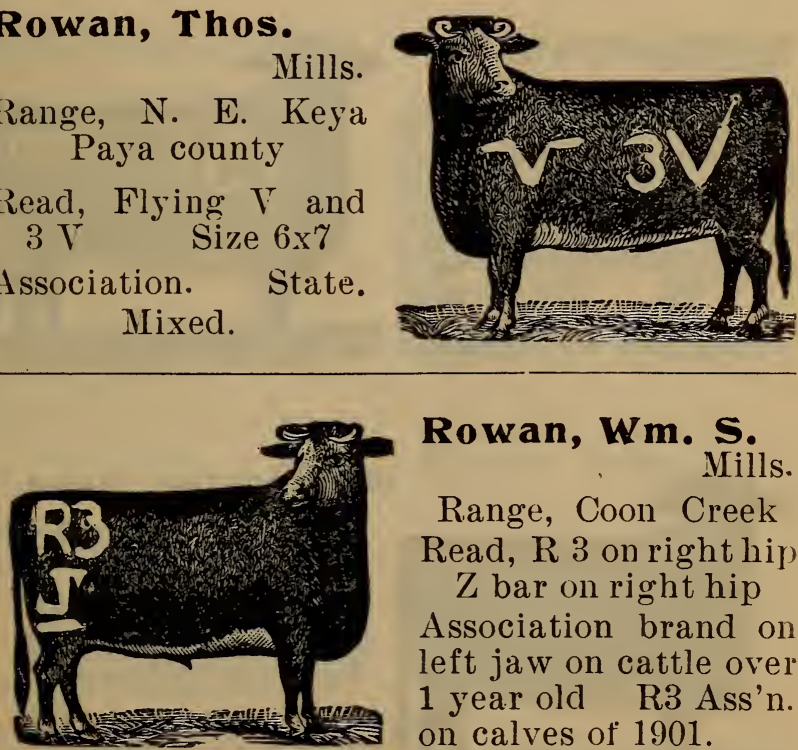

Rowan, Wm. S. Mills.

Range, Coon Creek Read, $\mathrm{R} 3$ on right hip $\mathrm{Z}$ bar on right hip Association brand on left jaw on cattle over 1 year old R3 Ass'n. on calves of 1901.

State. Association.

Mixed. 
Sylvester, Arthur Mills.

Range, 4 miles west of Mills

Read, Mxt connected Size $4 x 6$

County. ... Mixen.
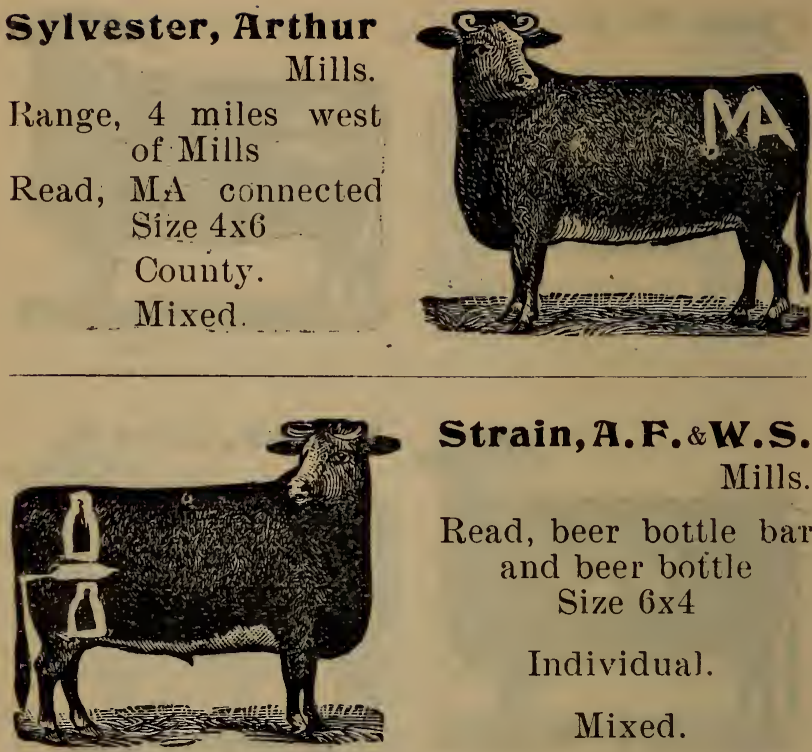

Strain, A.F.\&W.S. Mills.

Read, beer bottle bar and beer bottle Size $6 \times 4$

Individual.

Mixed.

Stroltenberg, A. Mills.

Range, between Coon and Spring Creeks

Read, Apple $\mathrm{S}$ and $\mathrm{Ox}$ Yoke Size $4 \times 5$

State. Ass'n.

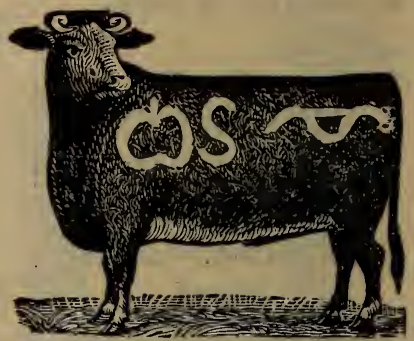

Sylvester, Cass Mills.

Range, $2 \frac{1}{2}$ miles west of Mills

Read, Flaring E Size $4 \times 6$

Individual. 
Smothers, $\mathrm{Wm}$.

Mills.

Range, Dry Creek

Read, bar Y S

Size $8 x y$

State.

Short Horn.

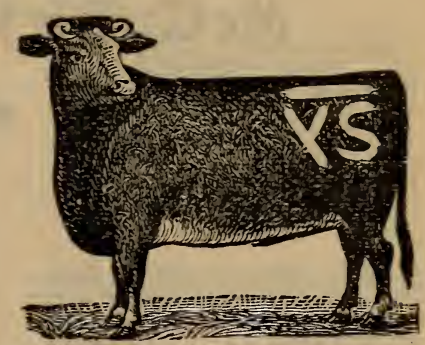

\section{Tuton, Nels.}

Mills.

Range, Keya Paha river

Read, You N Size 4xõ

Individual.

Common.

Wilkinson, Geo.W

Mills.

Range, Mills Precinct

Read, W bar right hip Individual.
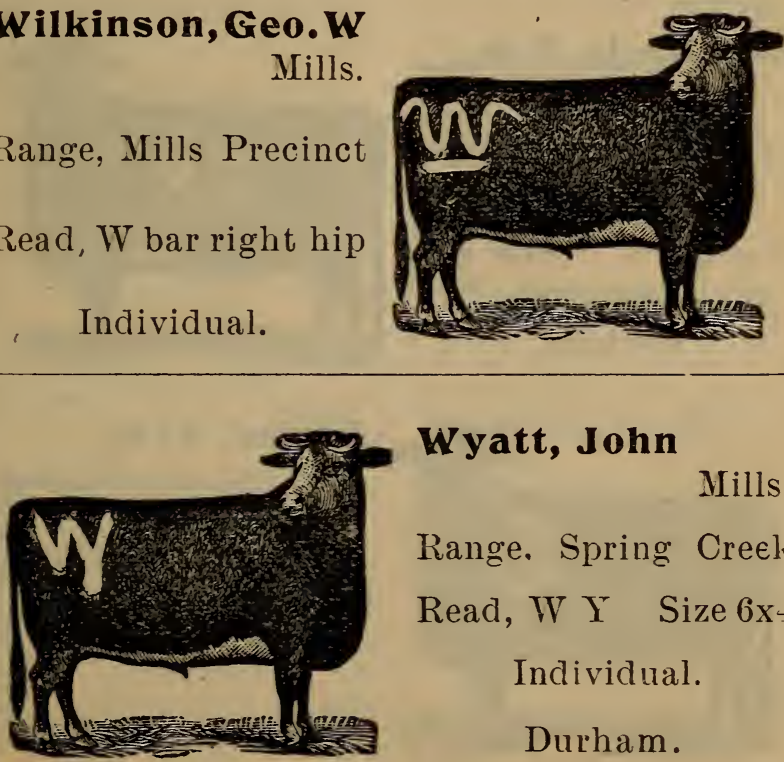

Wyatt, John

Mills.

Range. Spring Creek Read, W Y Size $6 \times 4$ Individual.

Durham. 


\section{McGuire Precinct.}

\section{Allen, M. M.}

Norden.

Range, Middle Creek and Niobrara River

Read, 3A

Size $4 \mathrm{x} 4$

Durham.

Polled Angus.

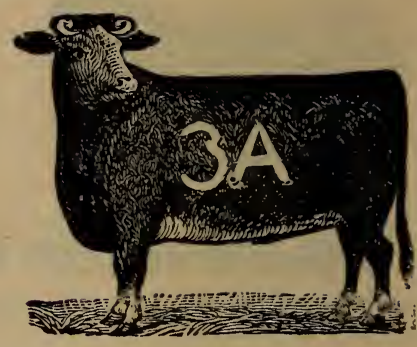

Bassett, W. E.

Norden.

Range, $1 \frac{1}{2}$ miles south Norden

Read, T J

Individual.

Mixed.

\section{Campbell, J. S.}

Norden.

Range, 2 miles west of Norden

Read, J S Size $4 \times 4$ Individual.

Durham.
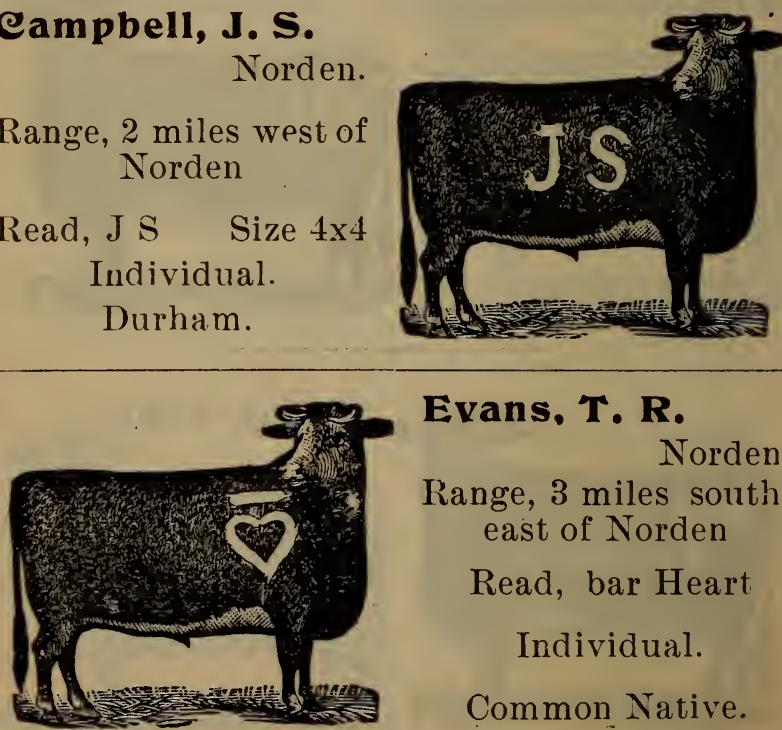

Evans, T. R.

Norden.

Range, 3 miles southeast of Norden

Read, bar Heart

Individual.

Common Native. 


\section{5}

Eglehoff, Fred

Norden.

Range, west end of County

Read, R E Size 4x5

Individual.

Mixed.
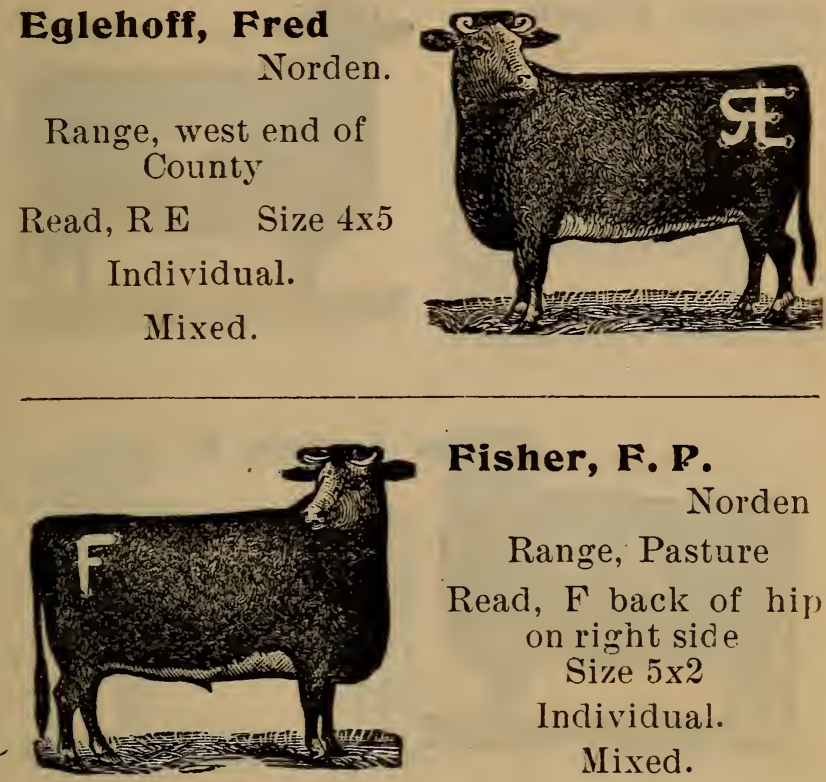

Fisher, F. P.

Norden

Range, Pasture

Read, F back of hip on right side

Size $5 \times 2$

Individual.

Mixed.

\section{Hutchinson, E. L.}

Penbrook.

Range, Niobrara river southwest of Norden

Read, $\mathrm{H}$ on neck right side Size $2 \times 3$

Individual.

Durham.
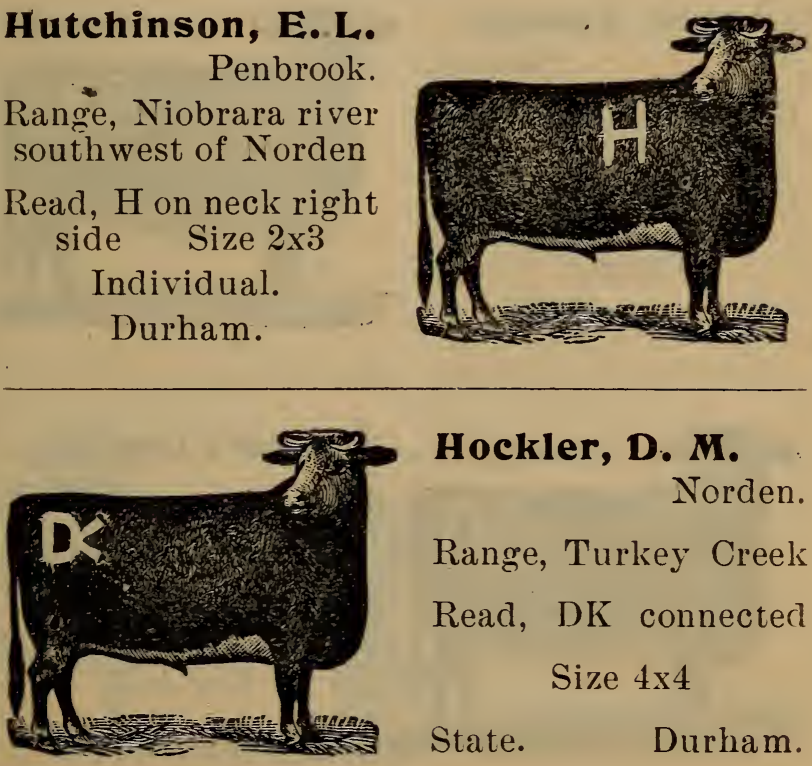

Hockler, D. M.

Norden.

Range, Turkey Creek

Read, DK connected

Size $4 \times 4$

State.

Durham. 
Kuhre, Frank

Norden.

Range, Niobrara river south of Norden

Read, TK Size 5x5

State. Hereford.
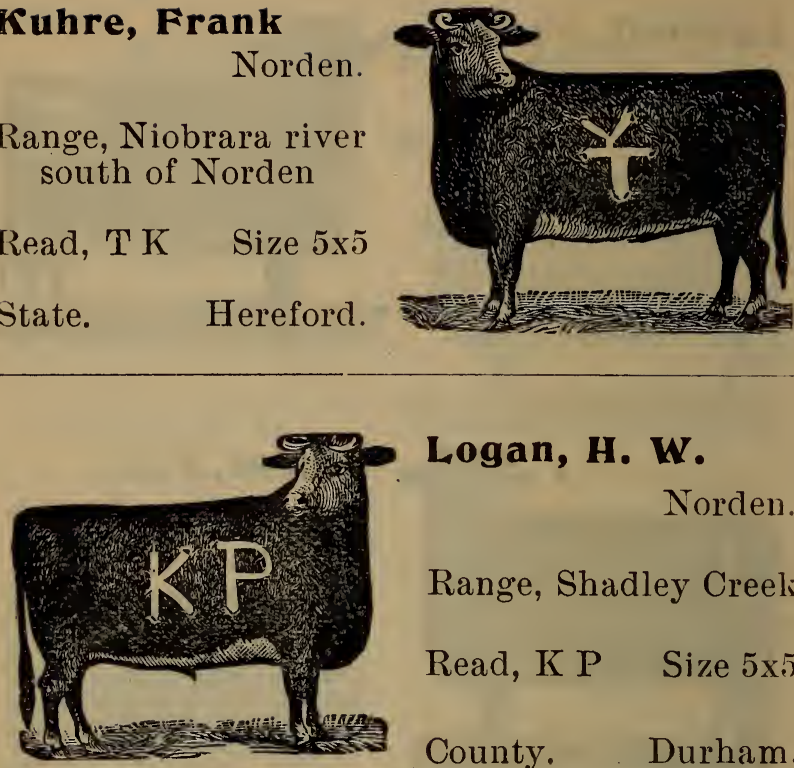

Logan, H. W.

Norden.

Range, Shadley Creek

Read, K P Size 5x5

County. Durham.

\section{Langer, Joseph}

Norden.

Range, 3 miles southwest of Norden

Read, S U Size $4 \times 6$

County.

Mixed.

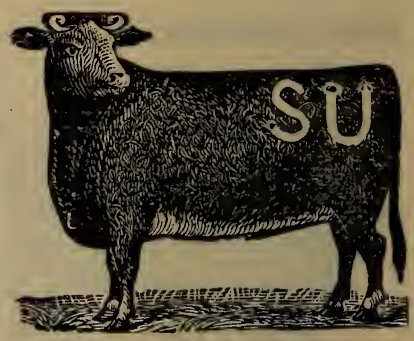

Langer, Louisa

Norden.

Range, 3 miles southwest of Norden

Read, S U inverted Size $4 x 6$

County. Mixed. 


$$
\text { ii }
$$

Laird, Thos.

Norden.

Range, Lost Creek

Read, T laxy I con-

nected Size $4 \times 4$

County.

Short Horn.

Horse brand 7 on left jaw.
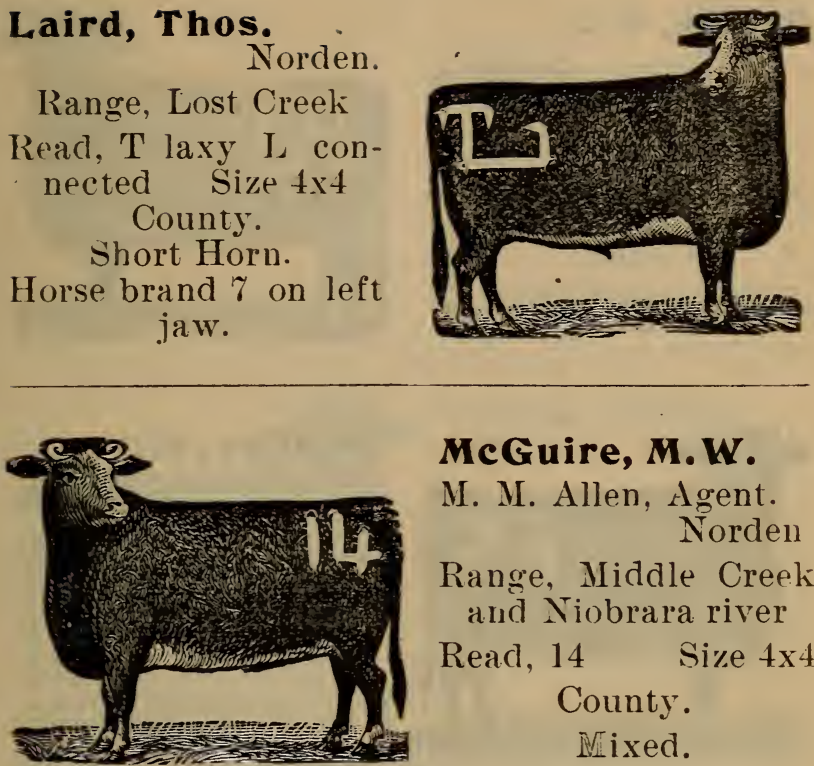

McGuire, M.W.

M. M. Allen, Agent.

Norden

Range, Middle Creek and Niobrara river

Read, 14 Size $4 \times 4$

County. $\mathbb{N}$ ixed.

\section{Morrison, F. N.}

Norden.

Range, west of Norden

Read, O bar $\mathrm{T}$ Size $3 x y$ Individual.

Mixed.

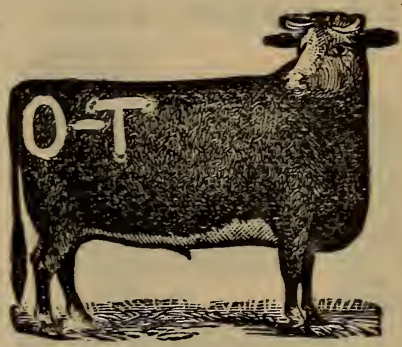

\section{Poitevin, Ant.}

Norden.

Range, west end of County

Read, la\%s $S \frac{1}{2}$ circle State. Mixed.

Old brand bar Heart on either hip. 
Porter, J. J.

Norden.

Range, Norden

read, JP J Size 5x

Cônty.

Durham.
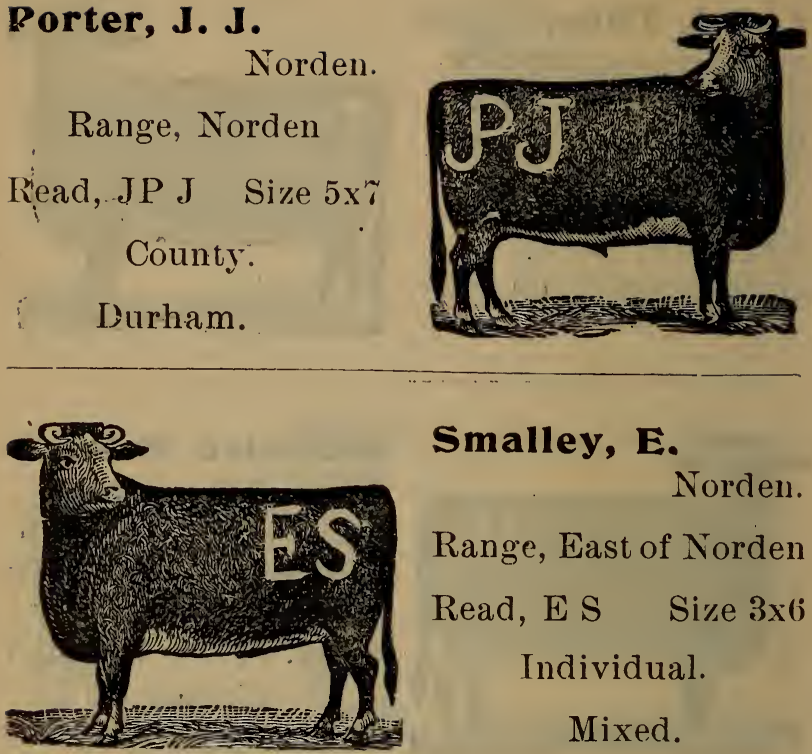

Smalley, E.

Norden.

Range, East of Norden

Read, E S Size $3 \times 6$

Individual.

Mixed.

\section{Thompson, John}

Norden.

Range, Niobrara south of Norden

Read, TK Size 5x5

State. Hereford.
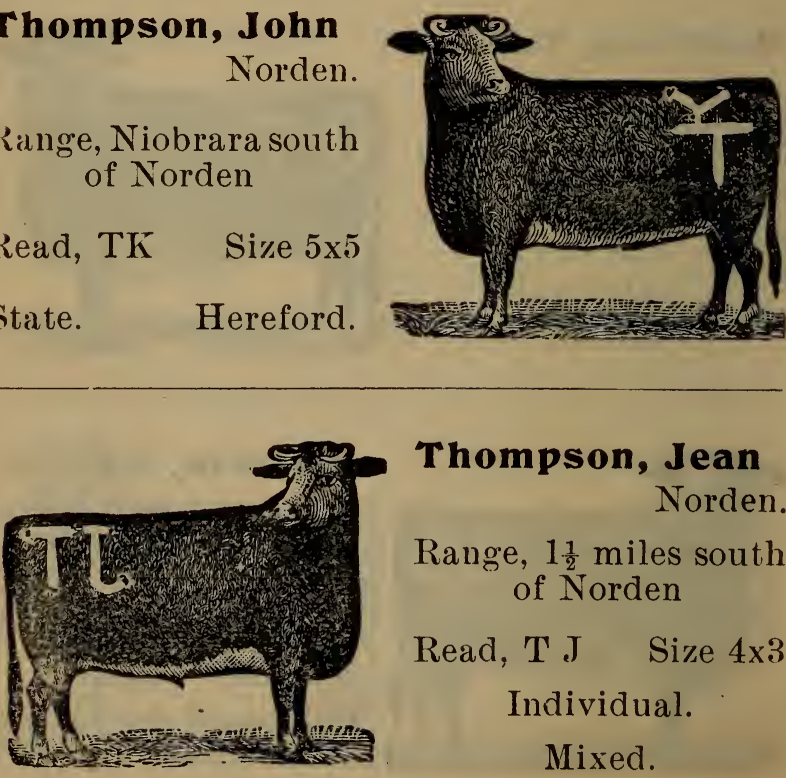

Thompson, Jean

Norden.

Range, $1 \frac{1}{2}$ miles south of Norden

Read, T J Size $4 \times 3$

Individual.

Mixed. 
Wells, J. B.

Norden.

Read, J Star Size $4 \times 7$

State.

Mixed.
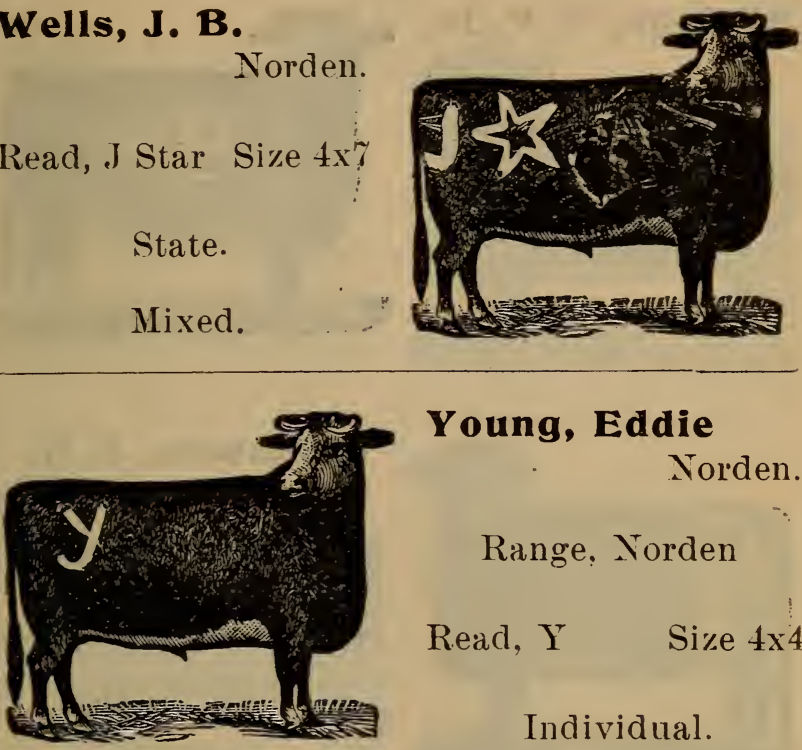

Young, Eddie

Norden.

\author{
Range, Norden \\ Read, Y Size $4 \mathrm{x} 4$ \\ Individual.
}

\title{
Norden Precinct.
}

Brewster, F. L.

Norden.

Range, Head of Mule Shoe Creek

Read, F B connected Size $5 \times 5$ on Calves State.

Durham.

Short Horn.
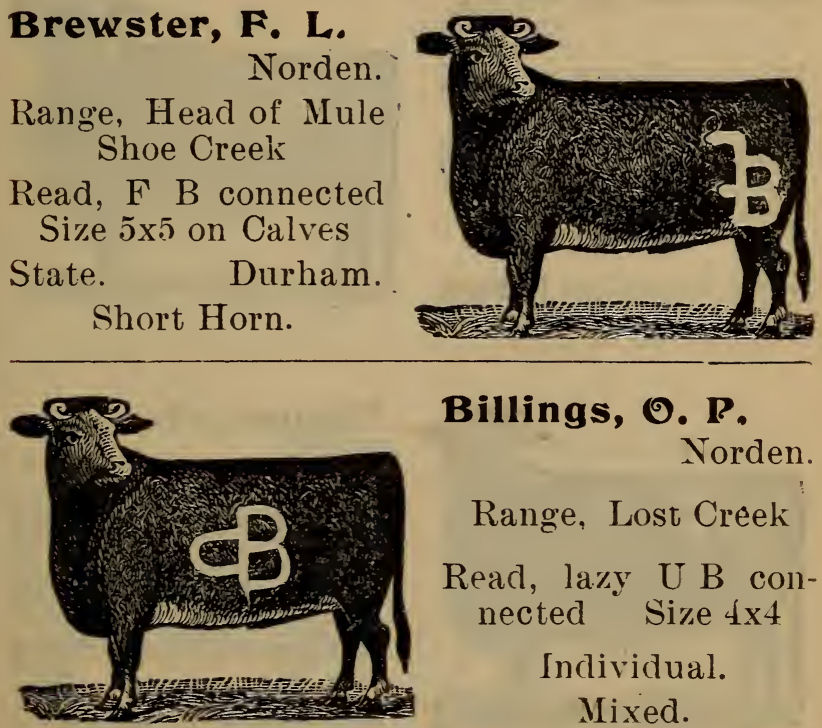

Billings, $\boldsymbol{O}$. P.

Norden.

Range, Lost Creek

Read, lazy U B connected Size $4 \times 4$

Individual.

Mixed. 
Billings, O. P. Jr.

Norden.

Range, Lost Creek

Read, B bar Size 6x20

Individual.

Short Horn.
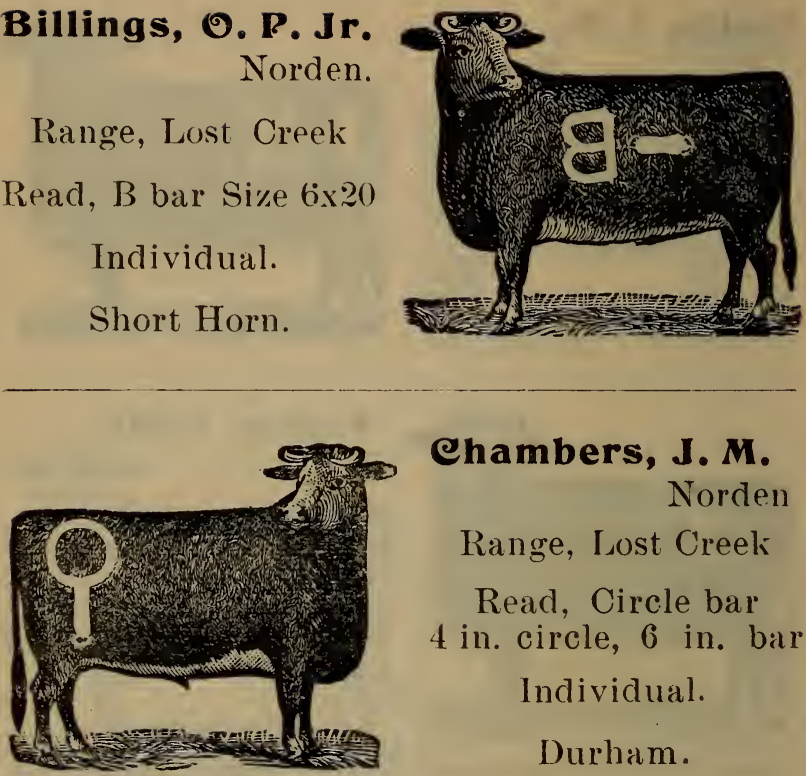

Chambers, J. M.

Norden

Range, Lost Creek

Read, Circle bar 4 in. circle, 6 in. bar Individual.

Durham.

Chambers, B. P.

Norden.

Range, Head of Lost Creek

Read, IX L Size 4x County. Mixed.

Same brand on horses.
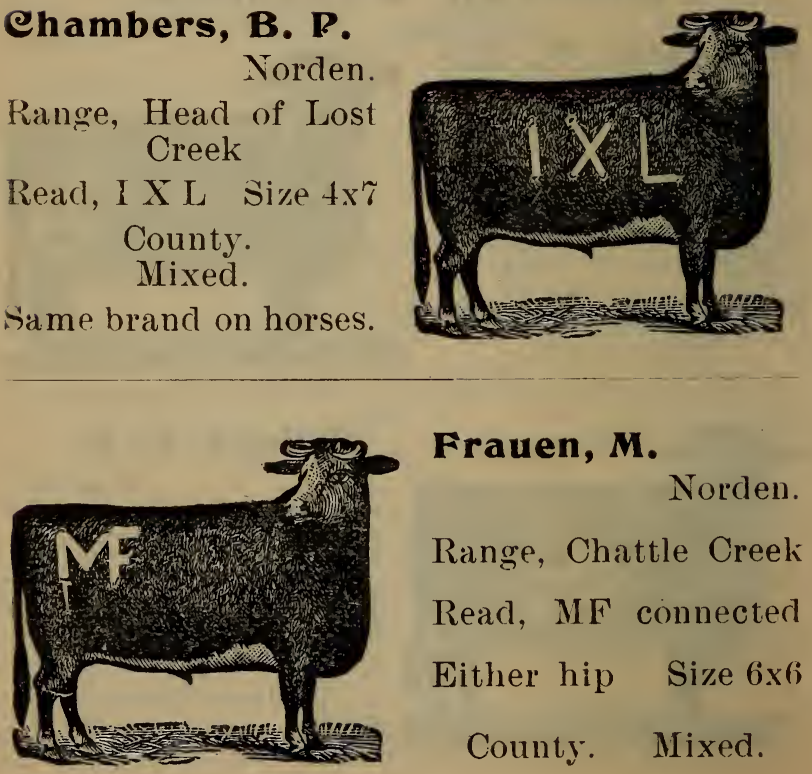

Frauen, $M$.

Norden.

Range, Chattle Creek

Read, MF connected

Either hip Size 6x6

County. Mixed. 


\section{1}

Ganow, Lewis

Norden.

Range, $3 \frac{1}{2}$ miles north of 'Norden.

Read, Circle bar and 7 H O Size 6x6

Individual. Durham.
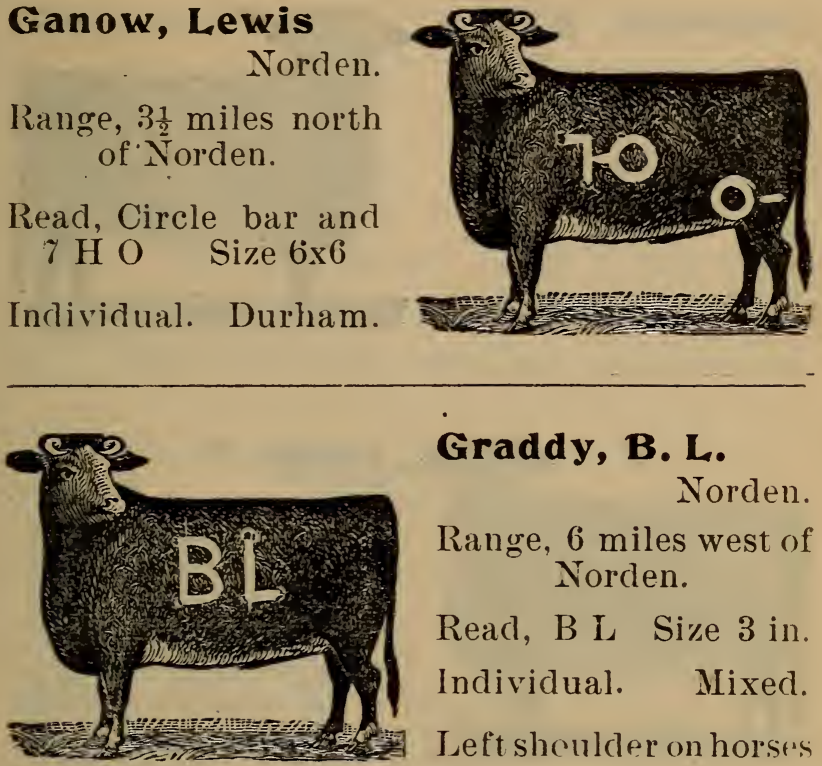

\section{Graddy, B. L.}

Norden.

Range, 6 miles west of Norden.

Read, B L Size 3 in. Individual. Mixed.

Left sheulder on horsess

Kirk, Jos. S.

Norden.

Range, Shadley Creek

Read, H J Size $6 \times 6$

County.

Mixed.
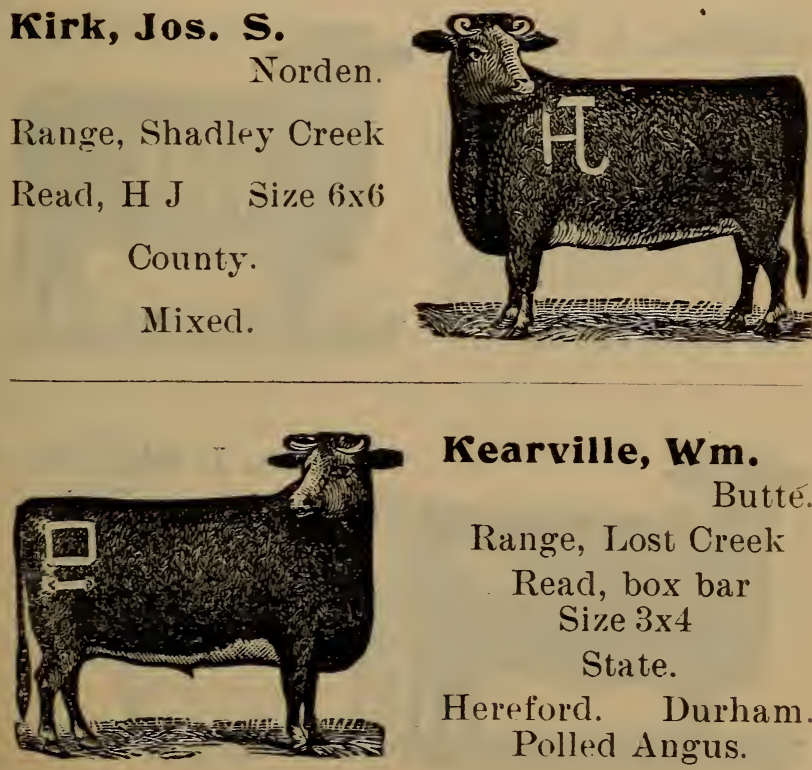

Kearville, $\mathrm{Wm}$.

Butté.

Range, Lost Creek

Read, box bar

Size $3 \times 4$

State.

Hereford. Durham.

Polled Angus. 
Lawrence, $A$.

Butte.

Range, North of

Norden

Size $7 \times 5$

Any part of body.

Mixed.
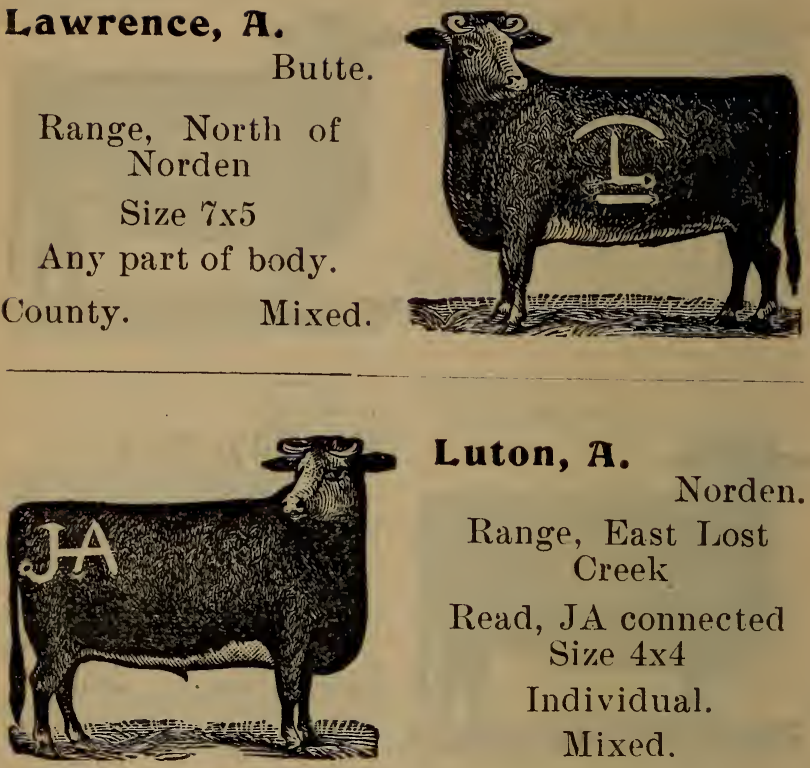

Luton, A.

Norden.

Range, East Lost Creek

Read, JA connected Size $4 \times 4$

Individual. Nixed.

Koplan, J. V. Norden.

Range, West of Lost Creek.

Read, 101 Size 4 in. Individual.

Short Horn.
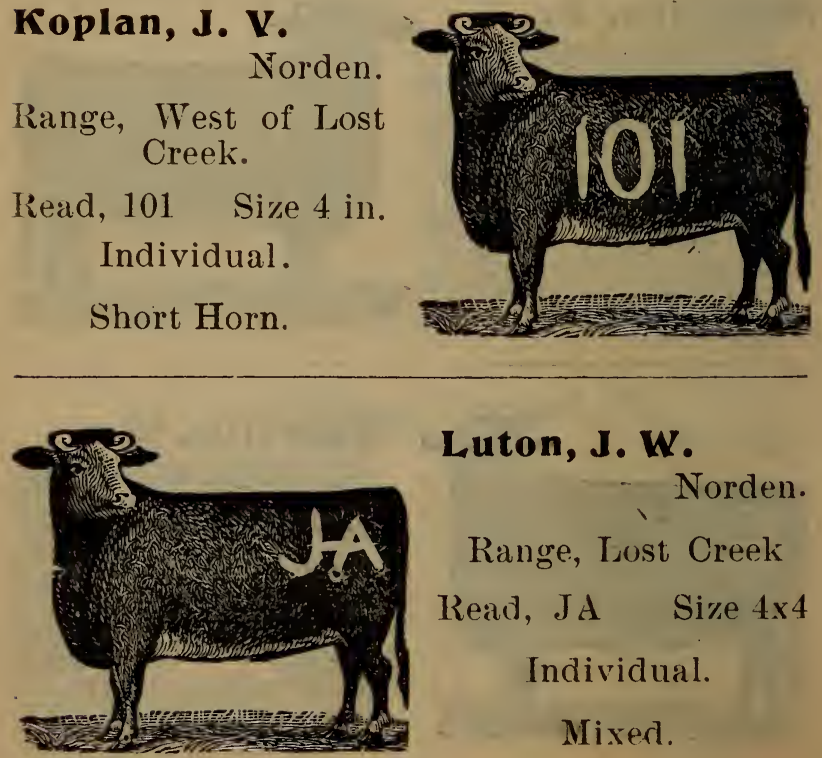

Luton, J. W.

Norden.

Range, Lost Creek

Read, JA Size $4 \times 4$

Individual.

Mixer. 


\section{3}

Moore, John S.

Norden.

Range, Lost Creek

Read, seven up, seven down under quarter circle. Size $3 \times 3$

County. Short Horn. Horse brand 7 on right

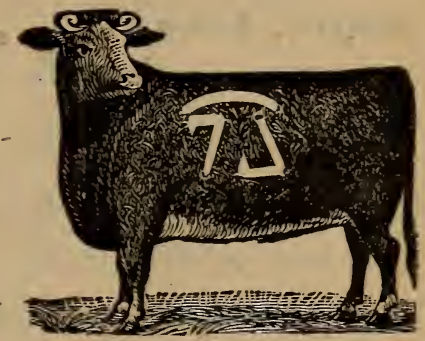
jaw, bar on right jaw, lazy 7 on left shoulder.

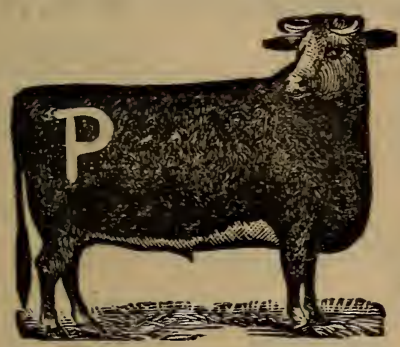

Powell, E. H. Norden.

Range, Cottonwood Creek

Read, $\mathrm{P} \quad$ Size 5x5

Mixed.

\section{Payne, Pearl}

Sparks.

Range, Shadley Creek Read, $\mathrm{P} \quad$ Size 4 in. Individual.

Holstein. Short Horn. Polled Angus.
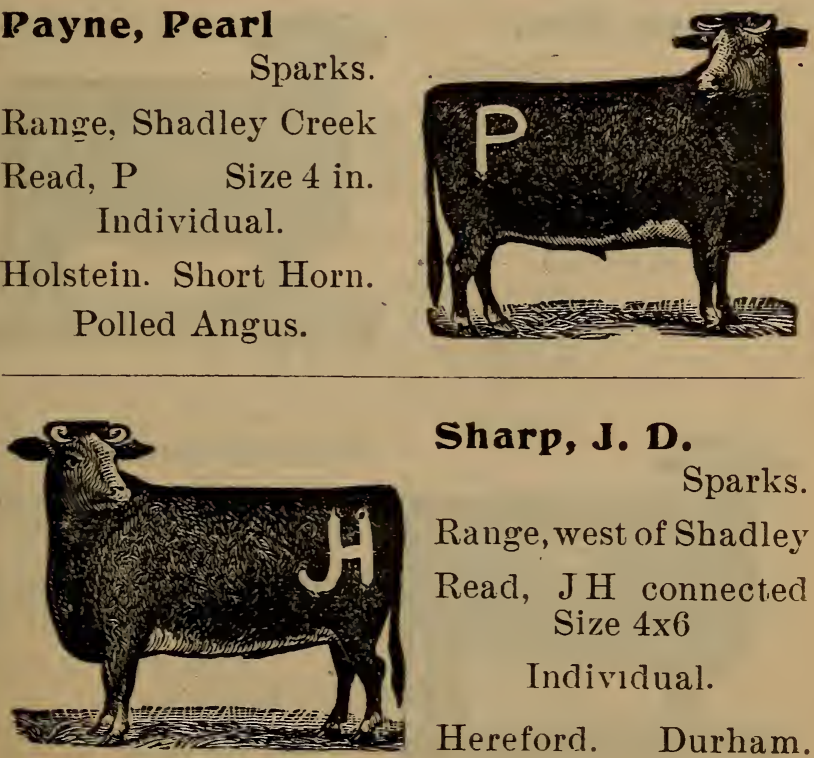

Sharp, J. D.

Sparks.

Range, west of Shadley Read, JH connected Size $4 \mathrm{x} 6$

Individual.

Hereford. Durham. 
Smith, John

Norden.

Range, Shadley Creek

Read, II on either hip

Also lazy $\nearrow_{4}$ on left hip

Mixed.
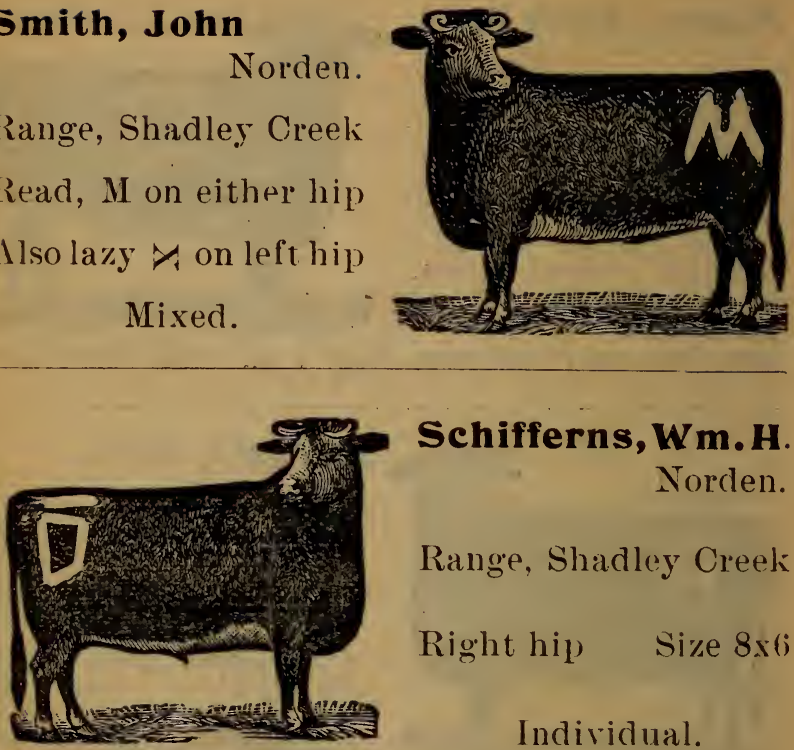

Schifferns, Wm.H.

Norden.

Range, Shadley Creek

Right hip $\quad$ Size $8 \times 6$

Individual.

\section{Siefken, Geo.}

Norden.

Range, Lost Creek

Rear, H R connected Size 5xร

State.

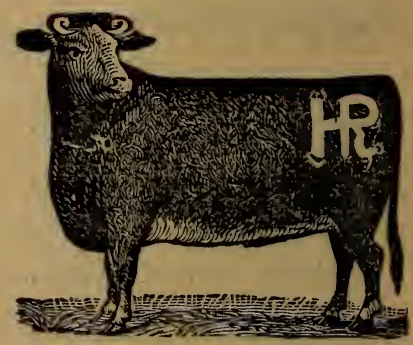

Starkjohann, Jno.

Norden.

Range, Rose Valiey Read, quarter circles

On Right Ribs Size $2 \times 4$ Individual.

Mixed. 


\section{Holstein-Fresians}

\section{THE DAIRY COW FOR THE WEST,}

\section{Breed the Best.}

Registered Stock at Moderate

Prices. Correspondence

Solicited.

HENRYY G, GLISSMAN

Station "B" R. D.

\section{Omaha, Neb.}

\section{GEORGE BROTHERS}

\section{PRINTING}

We can Print your Stationery and put Your Brand on the Animal.

Fraternity Building.

LINCOLN, - NEBRASKA. 
86

Amspoker, Fred Springview.

Range, Burton Creek

Read, $\mathrm{R} Y \quad$ Size $4 x 9$

State. Ass’n.

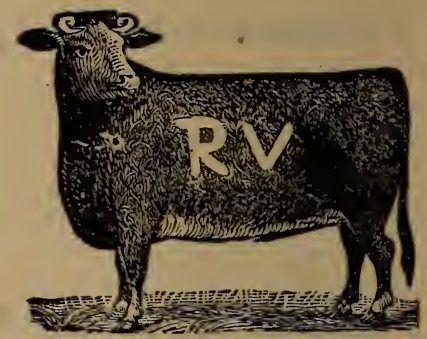

Amspoker, Frank Springview.

Range, Burton Creek Read, OA Size $7 \mathrm{x} 3$ Association. County.

Brede, Arthur

Range, near Carns Read, bar $\mathrm{B}$ and $\mathrm{T} B$ Size 6 in. long Gnly one brand on an animal Individual.

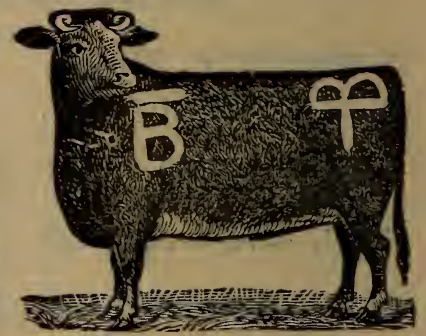

f

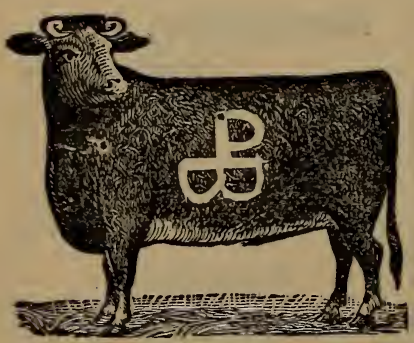

\section{Bahr, Peter}

Pine Camp.

Range, Wyman Creek Read, P B Size $4 \times 6$ State. Hereford.

Same brand for horses 
Cowger, J. T.

Springview.

Range, Spring Creek Read, J C Size 5x6 County.

Red Polled.
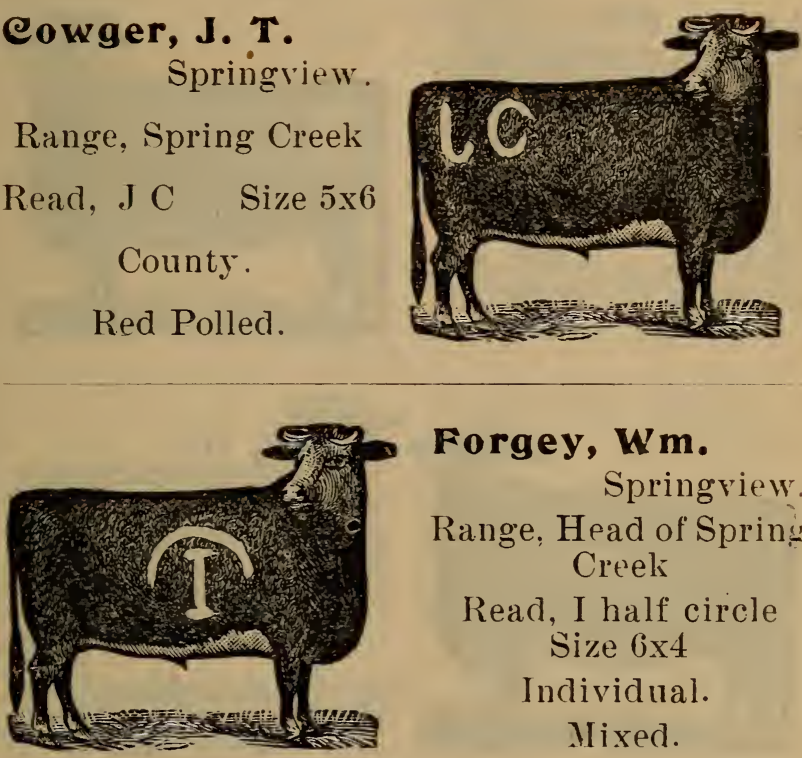

Forgey, Wm.

Springriew.

Range, Head of Spring Creek

Read, I half circle Size $6 \times 4$ Individual.

IIixed.

Gipson, Peter

Pine Camp.

Range, Pine Camp

Read, P G and T C

Size $2 \frac{1}{2} \times 3$

County.

Mixed.
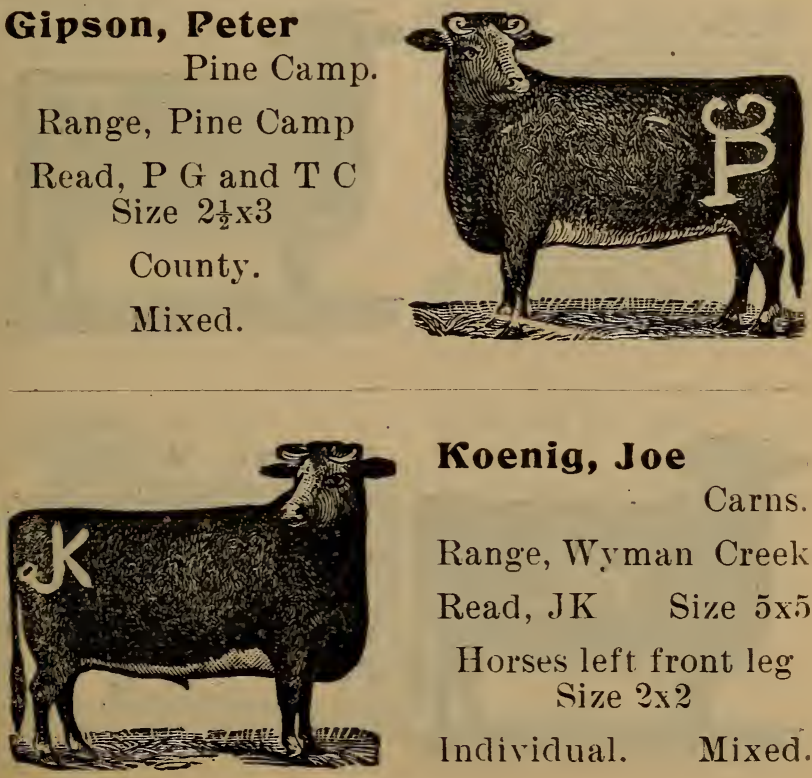

Koenig, Joe

Carns.

Range, Wyman Creek Read, JK Size 5x.5

Horses left front leg Size $2 x 2$

Individual. Mixed. 
Lowe, Chas.

Pine Camp.

Range, Spring Creek

to Simpson

Read, 2

Size $4 \times 4$

County. Mixed.
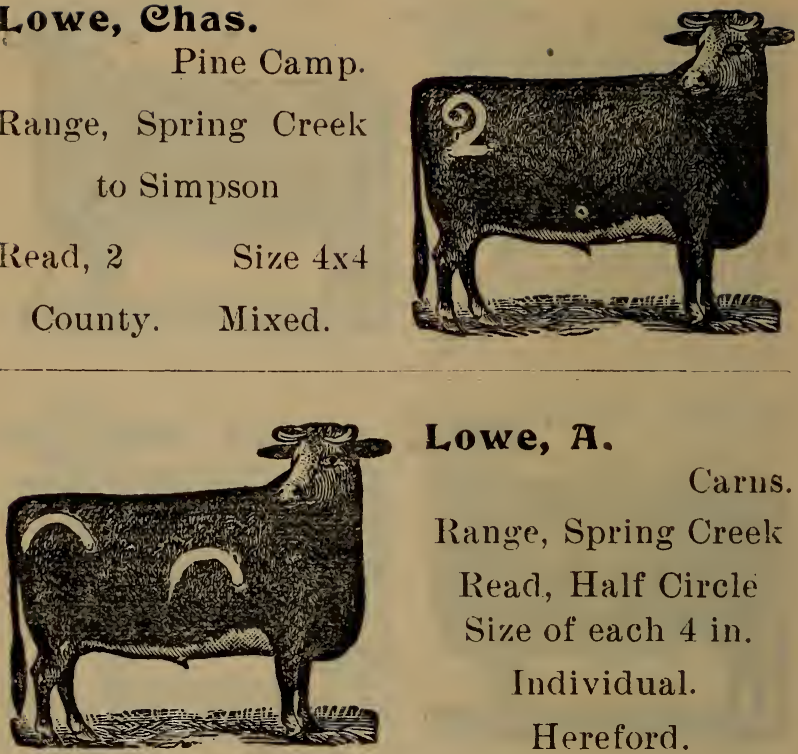

Lowe, A.

Carus.

Range, Spring Creek

Read, Half Circle

Size of each $4 \mathrm{in}$.

Individual.

Hereford.

\section{Mceracken, $\mathbf{R}$.} Pine Camp.

Range, Spring Creek Read, Heart Size $3 \times 4$ Individual.

Hereford.

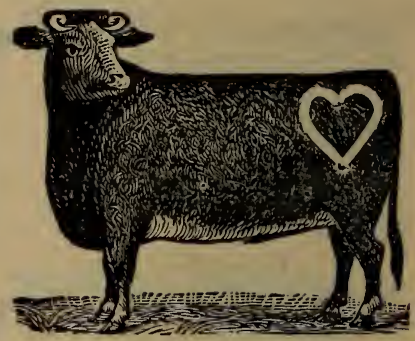

Osborn, W. L,

Springview.

Range, Wilkins Creek

Read, W O Size $5 \times 10$

Individual.

Durham. 
Oamek, Frank

Carns.

Range, 3 miles west Carns

Read, F O Size $6 \times 6$

Individual.

Mixed.
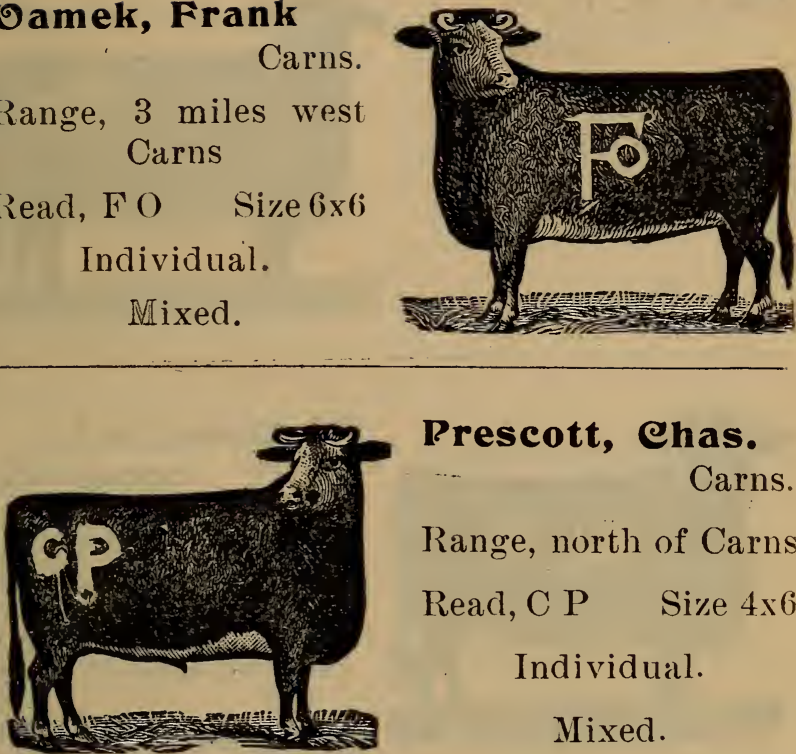

Prescott, Chas.

Carns.

Range, north of Carns

Read, C P Size $4 \times 6$

Individual.

Mixed.

\section{Paris, E. B. and Elsie $B$.}

Carns.

Range, $1 \frac{1}{2}$ miles southeast of Pine Camp

Read, P Size 5x3

Individual.

Hereford. Durham.

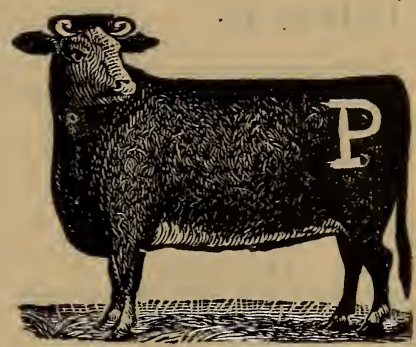

\section{Paris, R. B.}

Carns.

Range, Pine Camp

Read, $P \quad$ Size 4 in. Left thigh and back of shoulder Individual. Mixed. 
Peet, M. D.

Springview.

Range, Burton Creek Read, Shoe Size 5x5

Individual.

Durham.
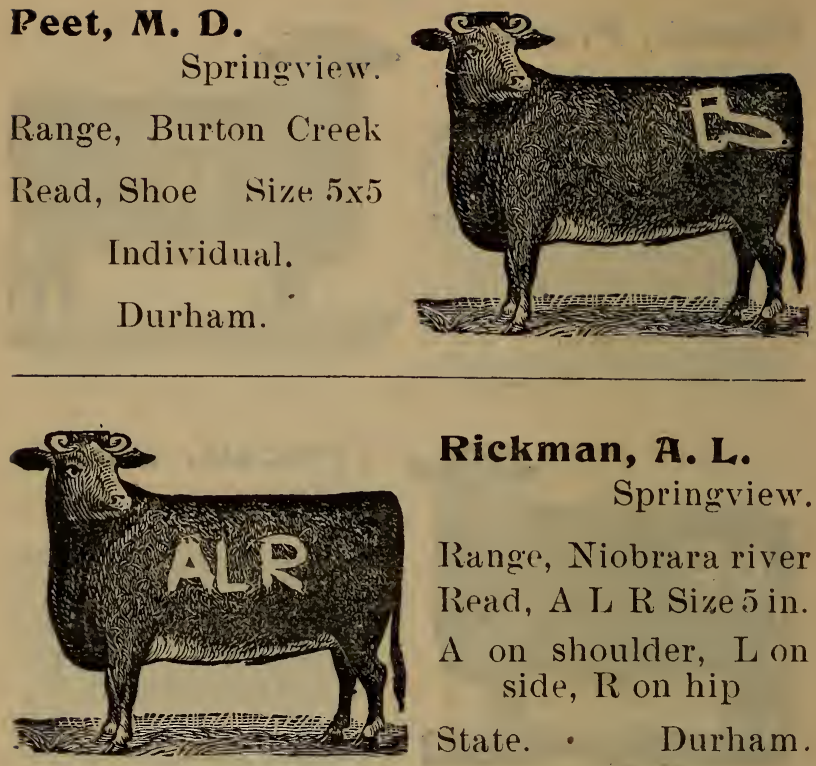

\section{Rickman, A. L.}

Springview.

Range, Niobrara river Read, A L R Size 5 in.

A on shoulder, L on side, R on hip

State. - Durham.

\section{Riley, E.}

Carns.

Range, Niobrara three miles west of Carns Read, 7 Sime $4 \mathrm{x}+$ Individual. Durham. Polled Angus.

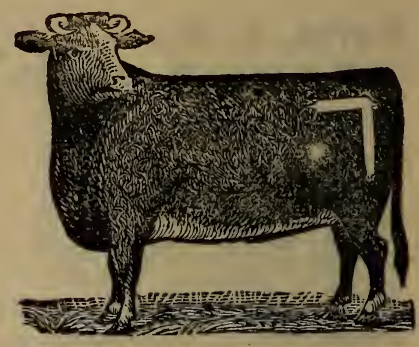

\section{Roe Bros.}

Carns.

Range, Niobrara river west of Carns

Read, S Z Size 5xi;

State.

Association. Mixed. 
Stewart, E. H.

Carns.

Range, Northwest of Carns.

Read, H S connected Size 5xว Individual. Hereford.
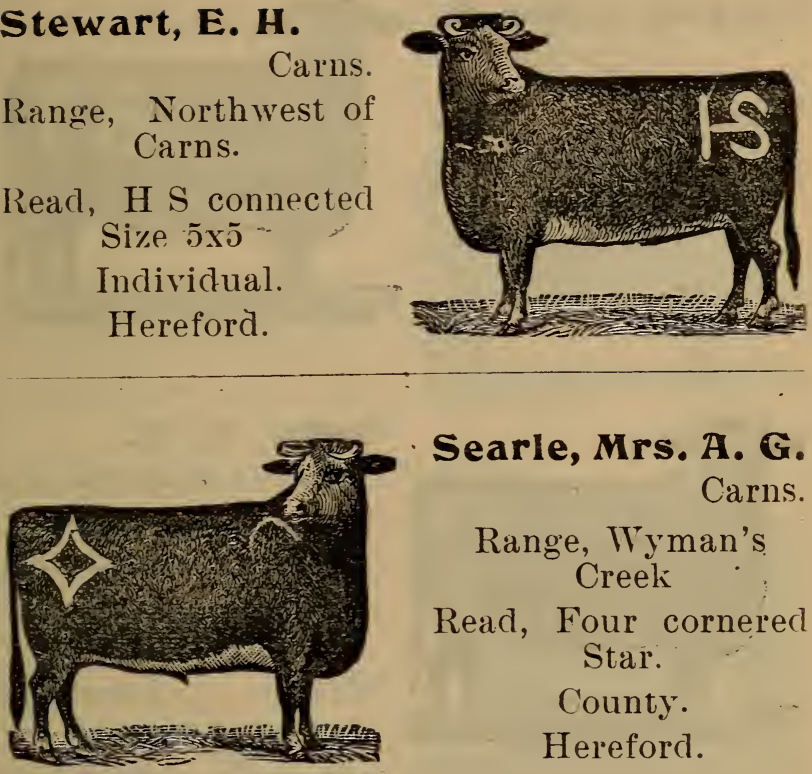

Searle, Mrs. A. G. Carns.

Range, Wyman's Creek

Read, Four cornered Star.

County. Hereford.

\section{Stewart, F. M. Carns.}

Range, Northwest of Carns

Read, S Size 5x3 Individual.

Durham. Hereford.

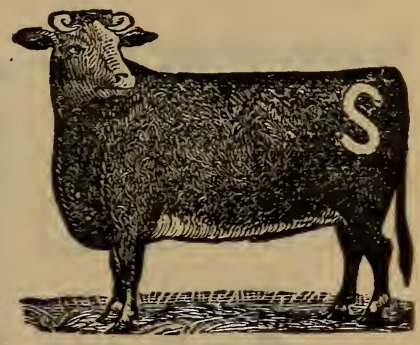

Seiga, Wm.

Carns.

Range, $2 \frac{1}{2}$ miles northwest of Carns

Read, JF Size $4 \times 6$

Also WS on right hip County.

Hereford. 


\section{2}

\section{Shepherd, Geo. M.}

Springview.

Range, Burton Creek

Read, B $7 \quad$ Size $5 \times 6$

County.

Hereford.
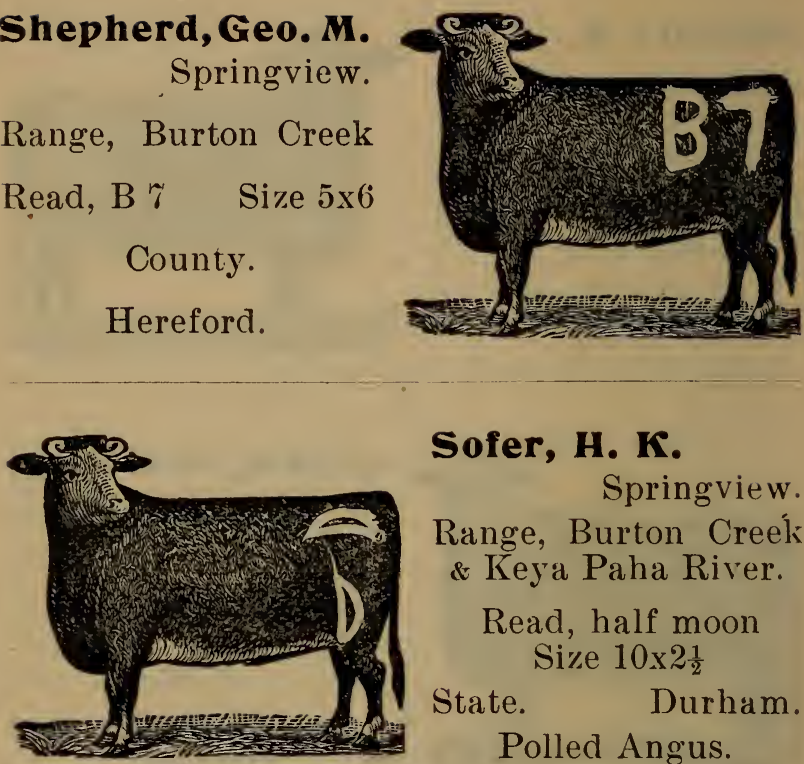

Sofer, н. к.

Springview.

Range, Burton Creek \& Keya Paha River.

Read, half moon Size $10 \times 2 \frac{1}{2}$

State.

Durham.

Polled Angus.

Same brand on right shoulder of horses single

Wentworth, A. A. Pine Camp.

Range, Niobrara river (i miles west of Carns Read, AW Size $3 \times 4$

State.

Mixed.
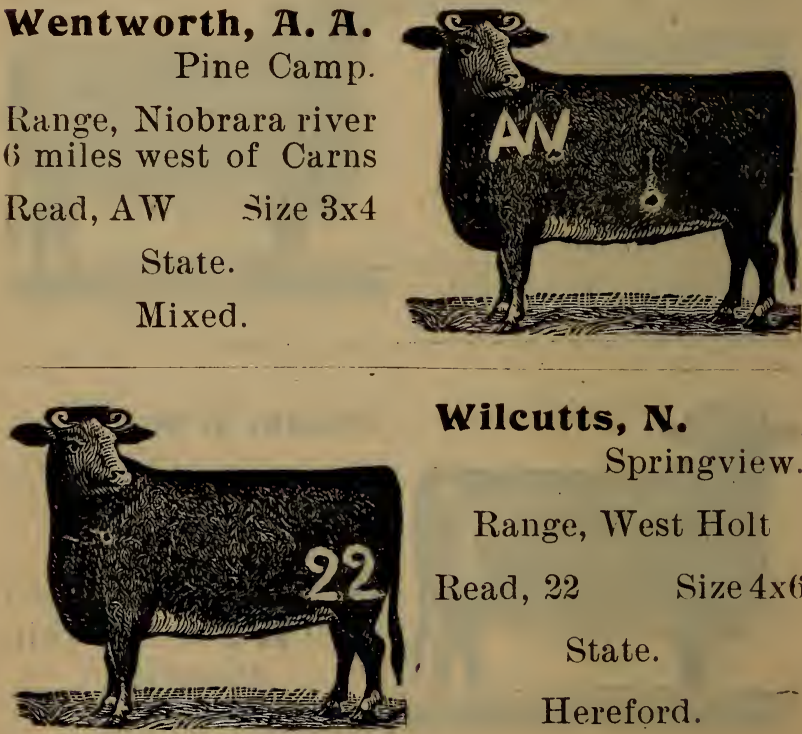

Wilcutts, $\mathbf{N}$.

Springview.

Range, West Holt

Read, $22 \quad$ Size $4 \times 6$

State.

Hereford. 


\section{Simpson Precinct.}

\section{Brown, Solon}

Simpson.

Range, Southwest of King Hill

Read, JA B connected Size 5x5

Horses left shoulder Individual. Durham.

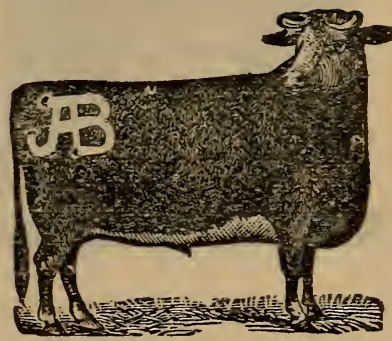

Brown, Herbert

Simpson.

Range, Runon Creek Read, JAB connected Size 5x 5

Horses same brand on left shoulder

Individual. Durham.
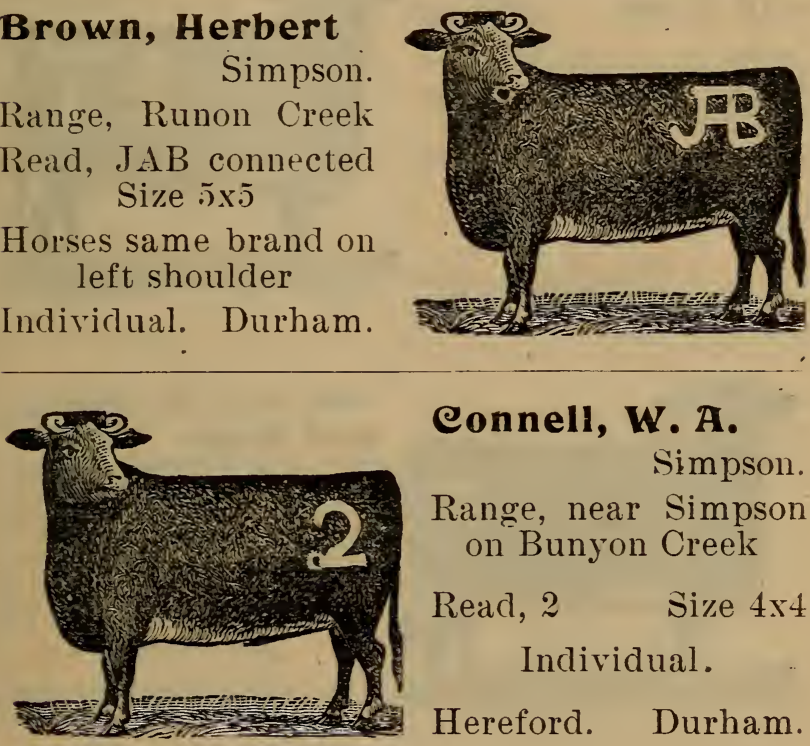

Connell, W. A.

Simpson.

Range, near Simpson on Bunyon Creek

Read, $2 \quad$ Size $4 \times 4$ Individual.

Hereford. Durham.

\section{Dyer, John M.}

Carns.

Range, 5 miles below Carns.

Read, Tuyer Iron Size $6 \times 6$

County. Short Horn. Also brisket wattle mark.

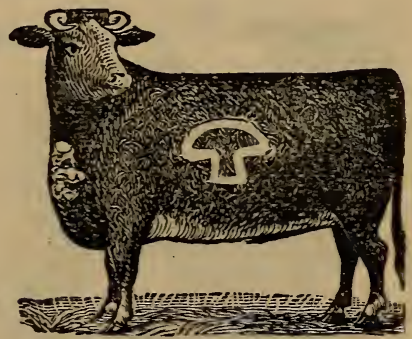


94

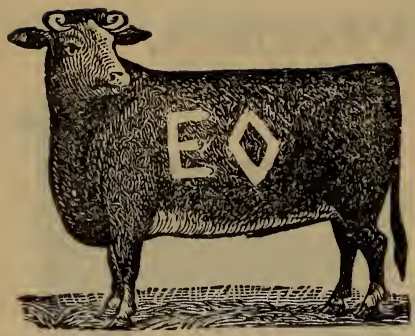

Elen, e. J.

Mills.

Range, 6 miles south of Mills

Read, E diamond

Size $10 x 8$

State. Mixed.

\section{Genung, L. $T$.} and Sons

Carns.

Range, N. E. of Carns Read, L T Size 6x6

State.

Mixed.
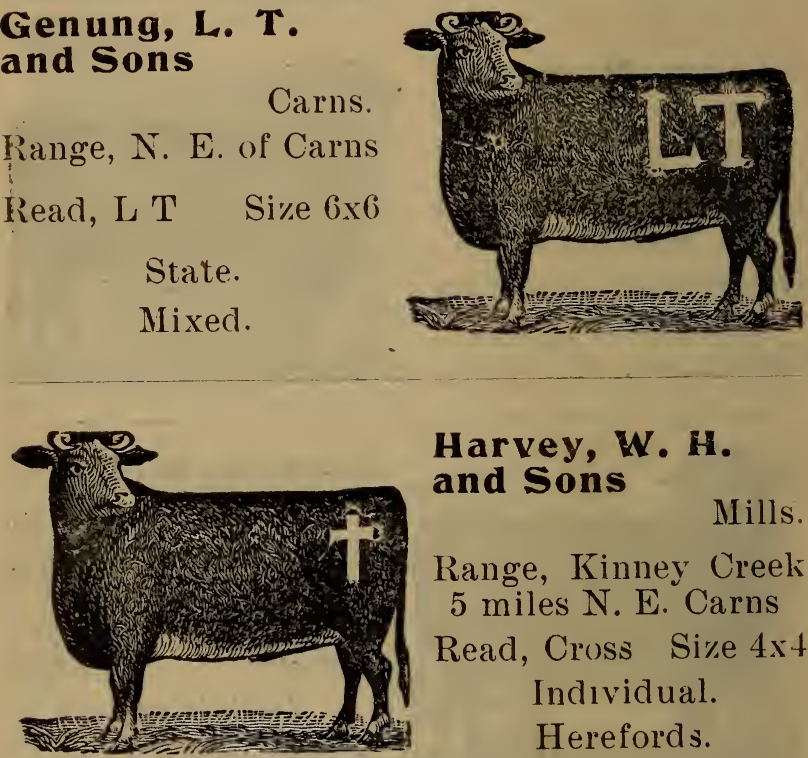

Harvey, W. H. and Sons

Mills.

Range, Kinney Creek 5 miles N. E. Carns

Read, Cross Size $4 x+$ Individual. Herefords.

\section{Hougen, $\mathbb{P}$.}

Simpson.

Range, about Simpson

Read, 96

Association.

State.

Mixed.

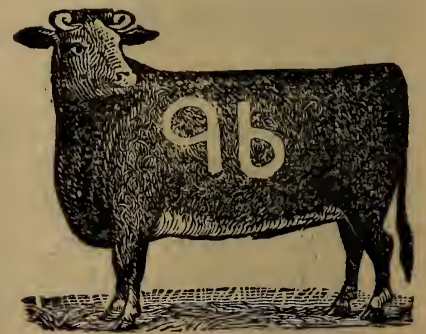




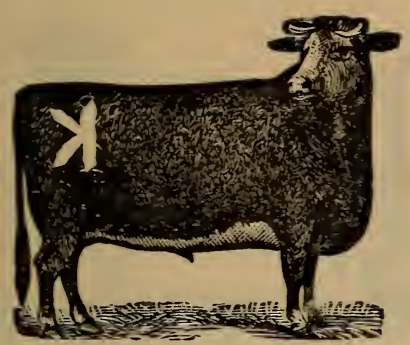

Koburg, Fred $M$.

Simpson.

Range, 2 miles west of Simpson.

Read, $K$ on right hip. Size $4 \times 3$

Individual.

Ass'n.

Mixed.

Swaffer, e. J.

Simpson.

Range, Pleasant Talley

Read, Double Wrench

Size $12 \mathrm{in.}$ long

State. Association. Hereford. Durham.
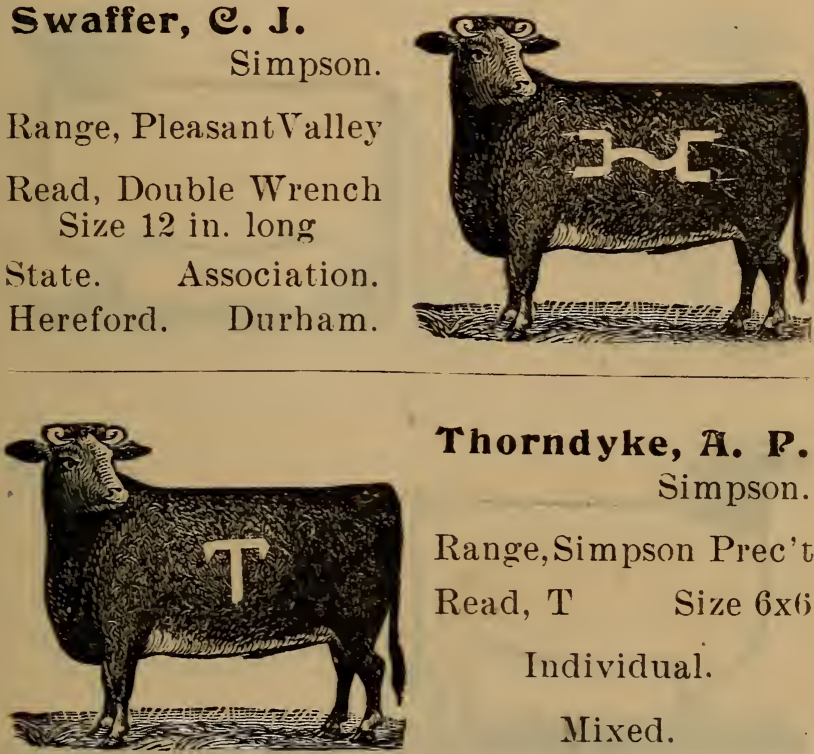

Thorndyke, A. P. Simpson.

Range,Simpson Prec't Read, T Size $6 \times 6$ Individual. Mixed.

\section{Turney, B. S.}

Simpson.

Range, 1 mile west of Simpson

Read, T B Size 5xร Individual. Hereford.

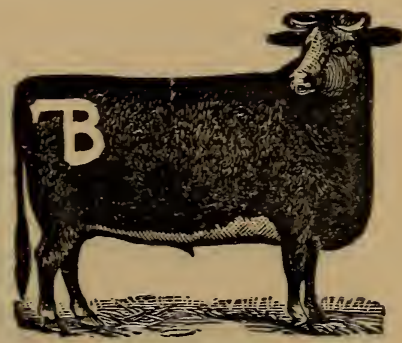



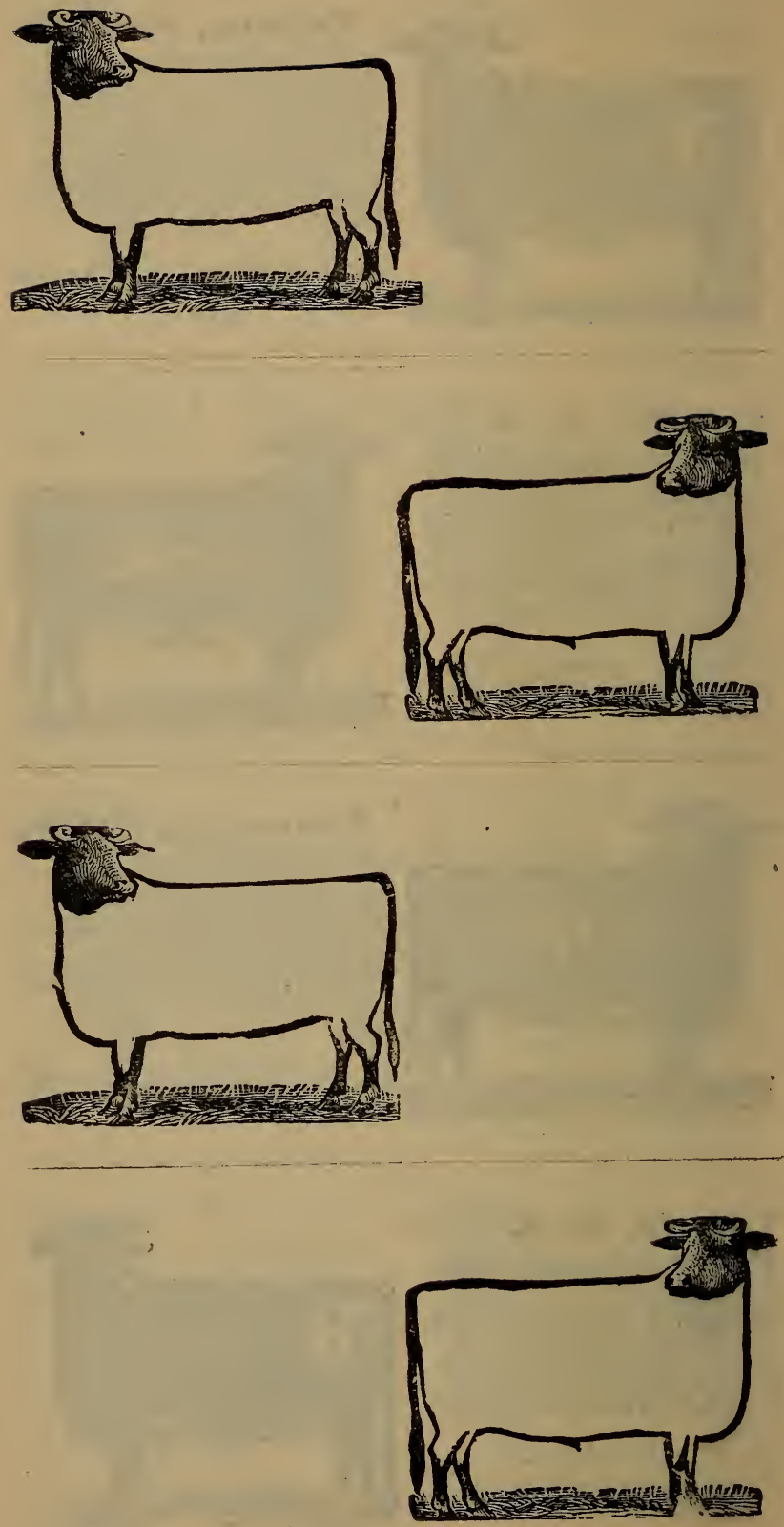

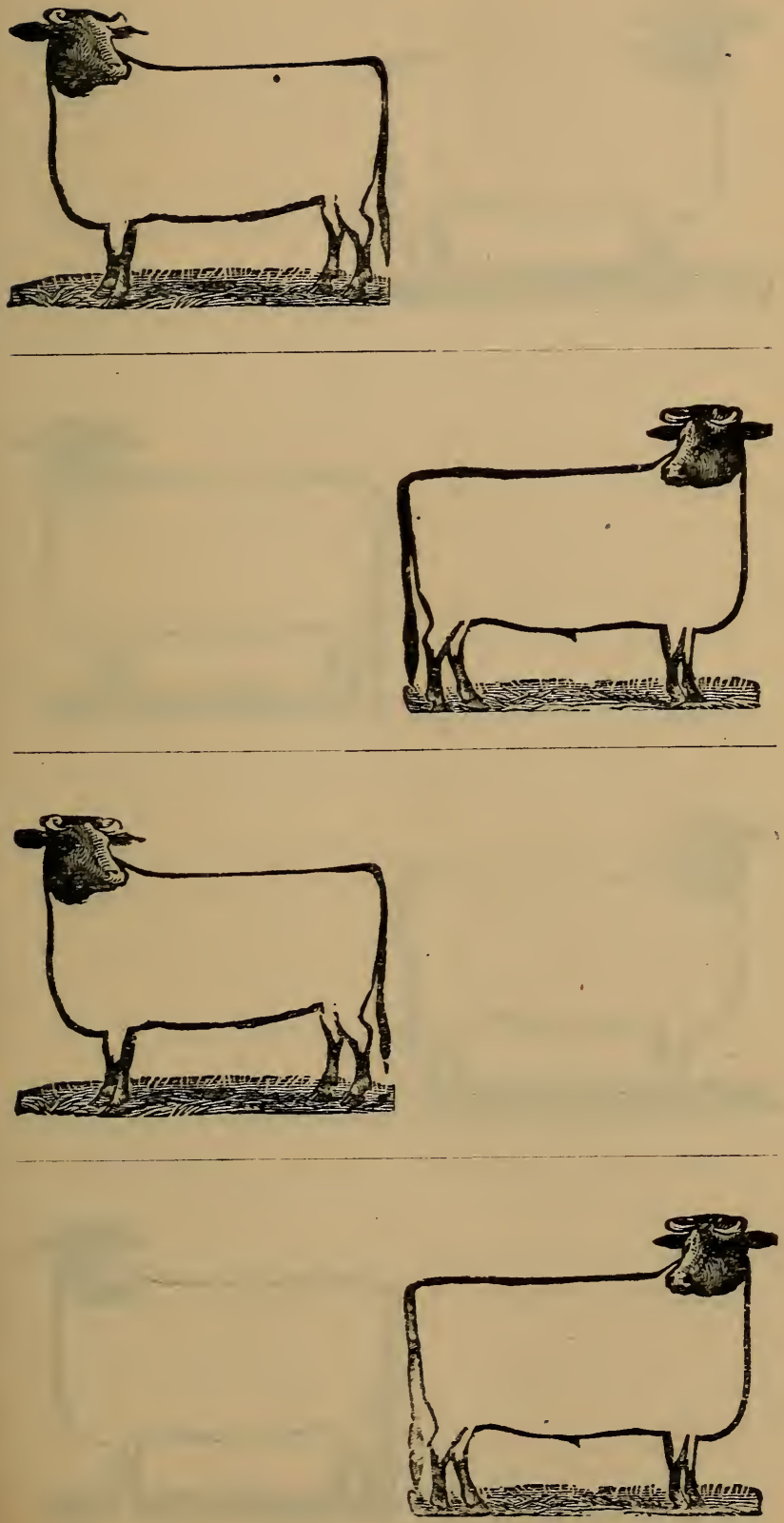

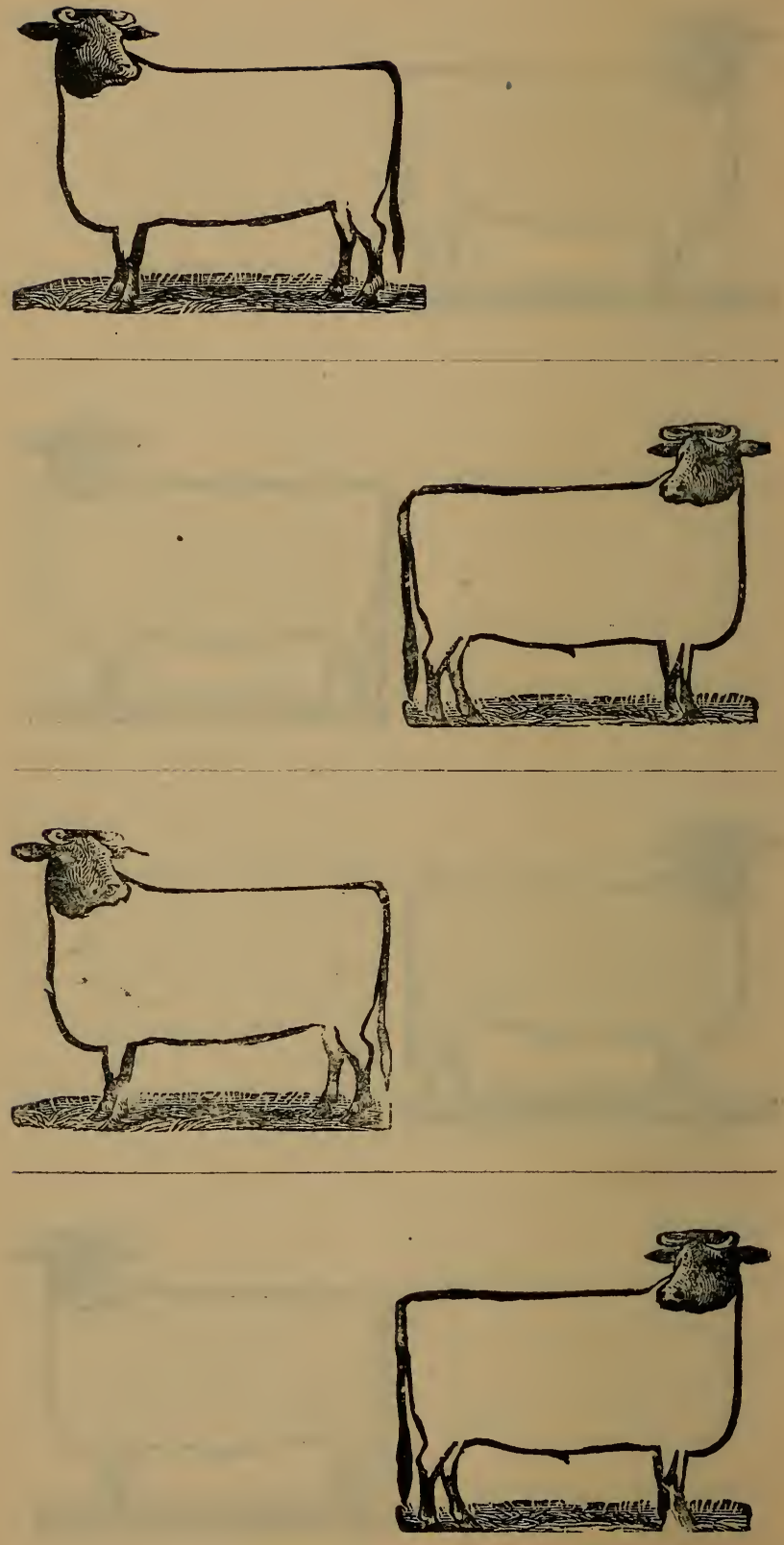

$\checkmark 19$ 

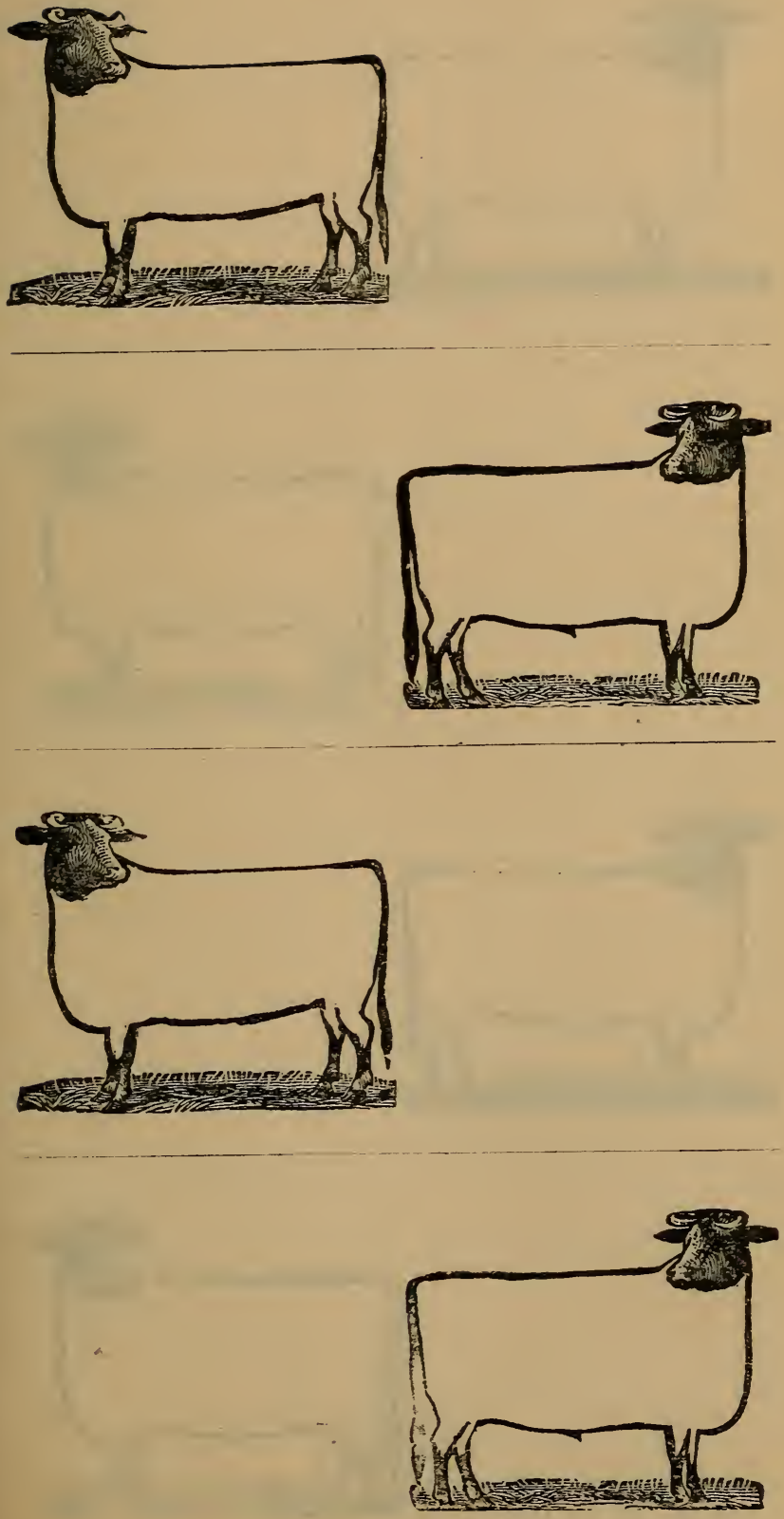

L. of C. 

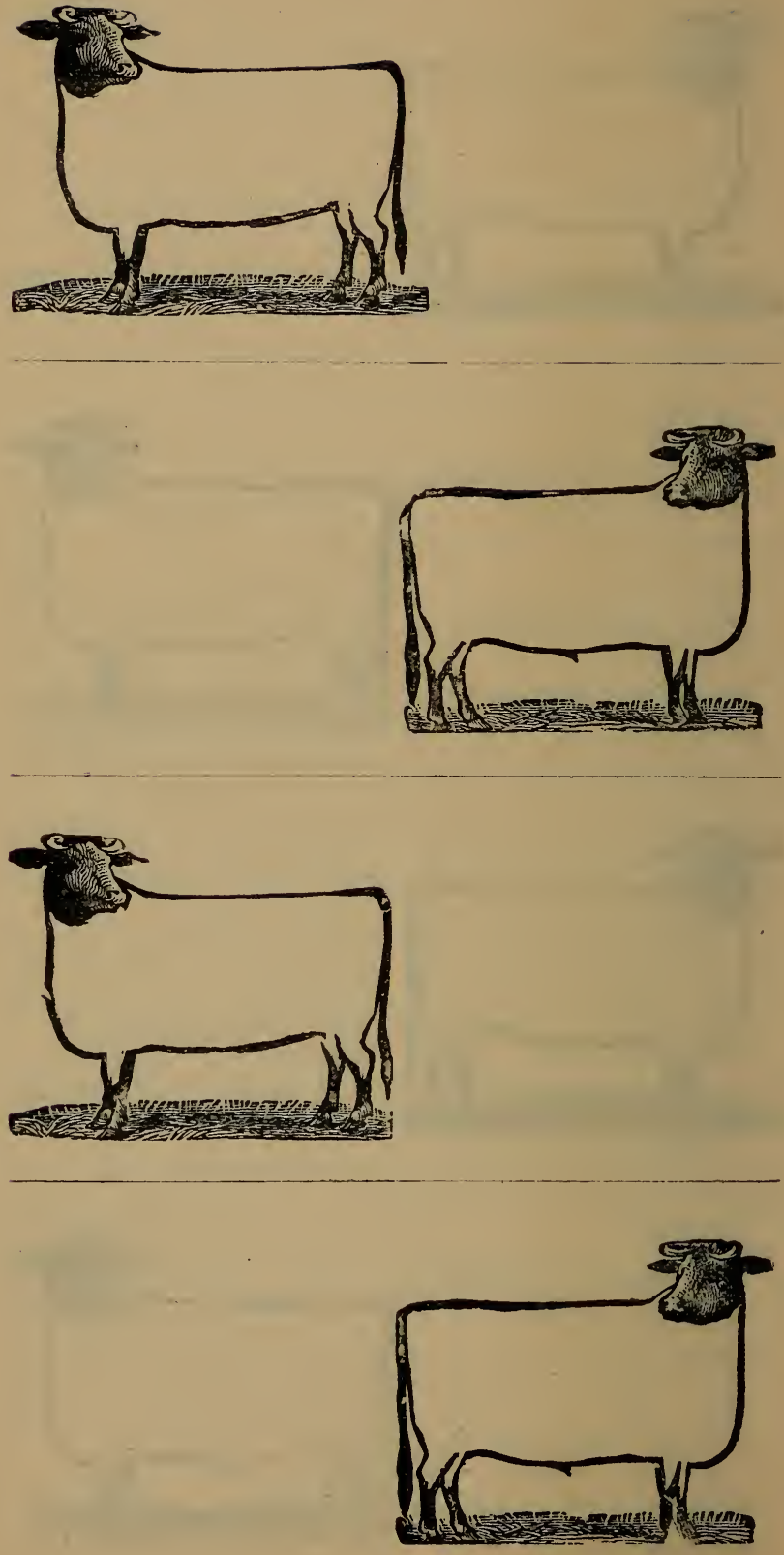

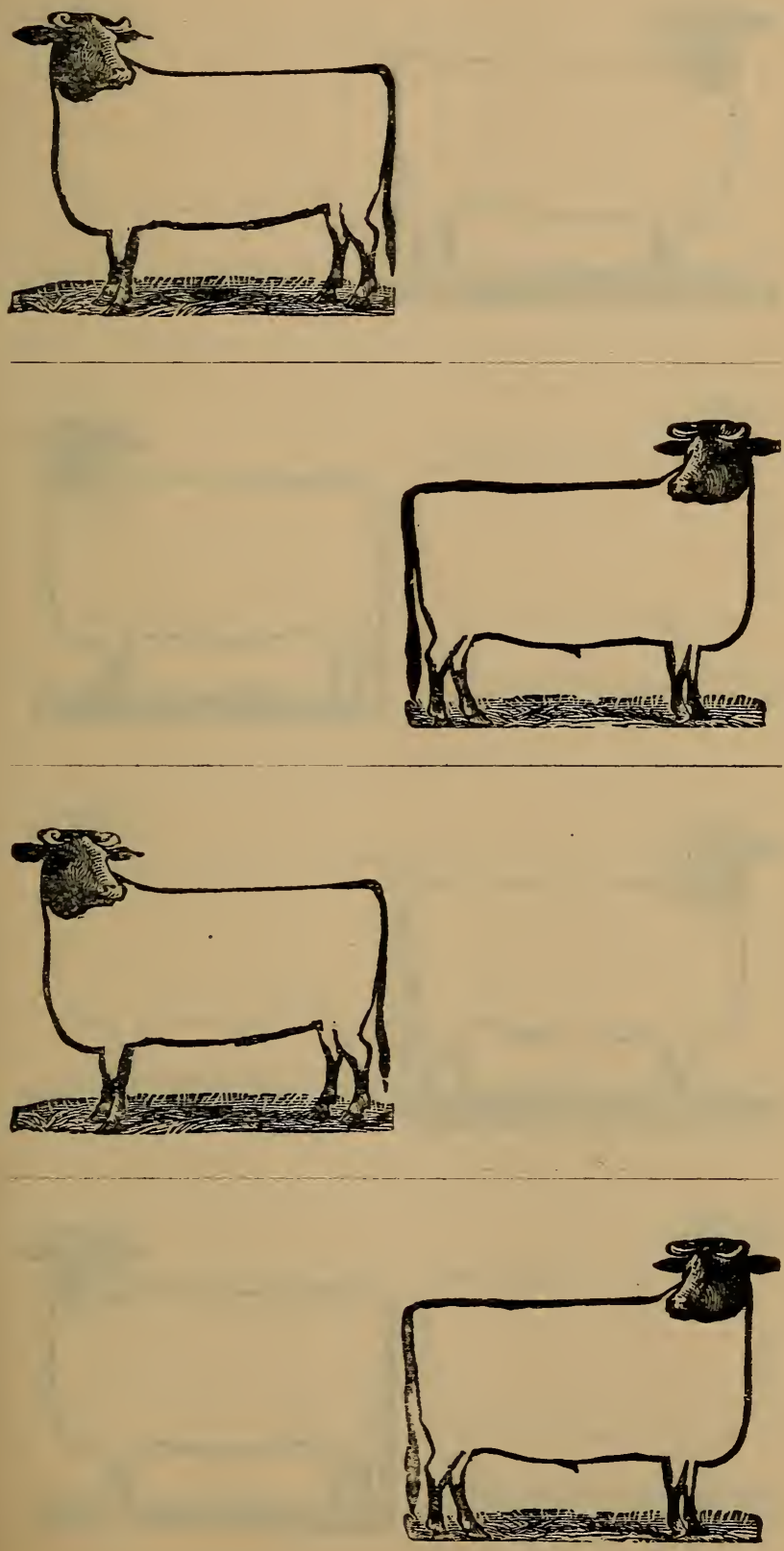

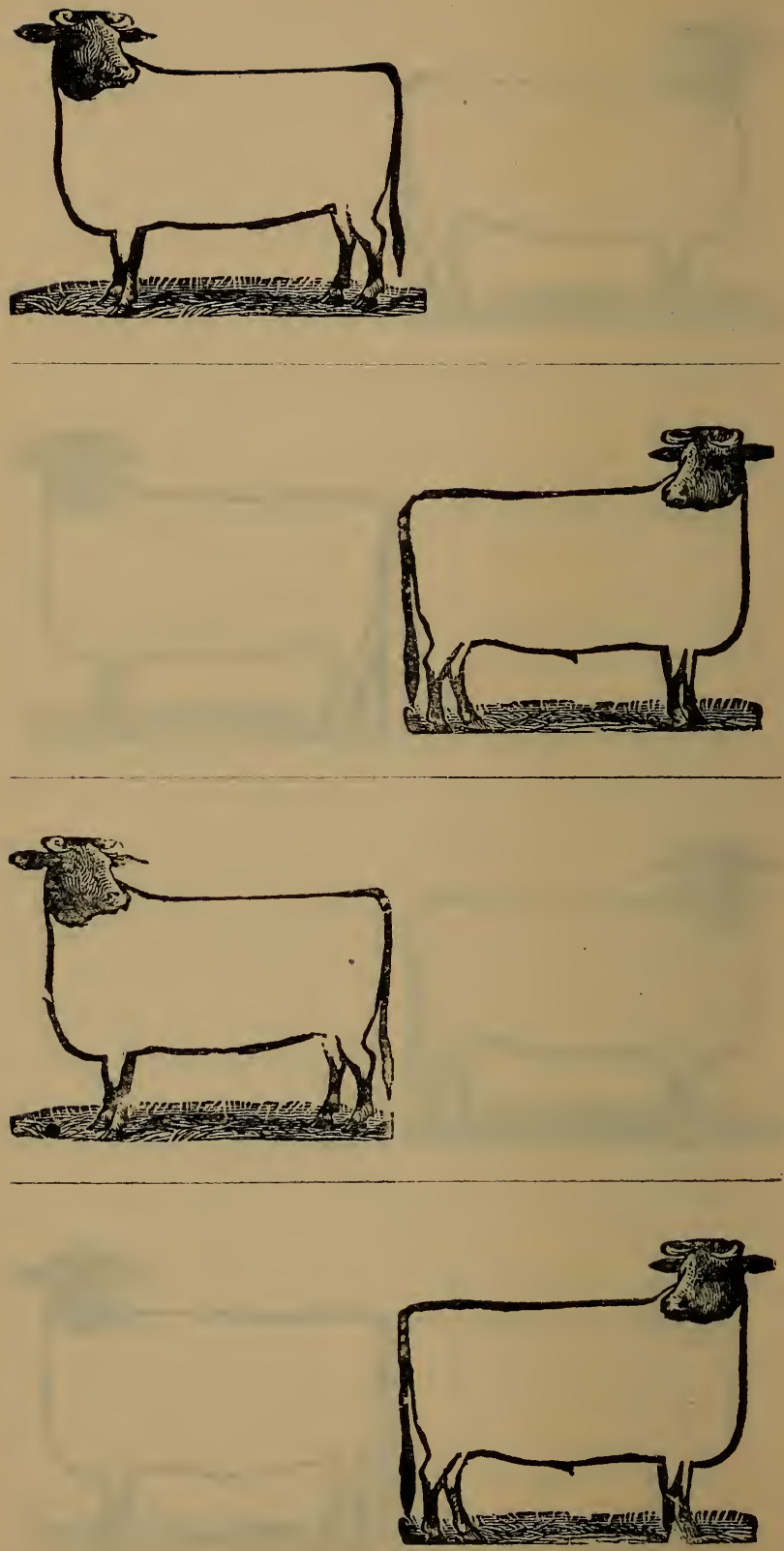

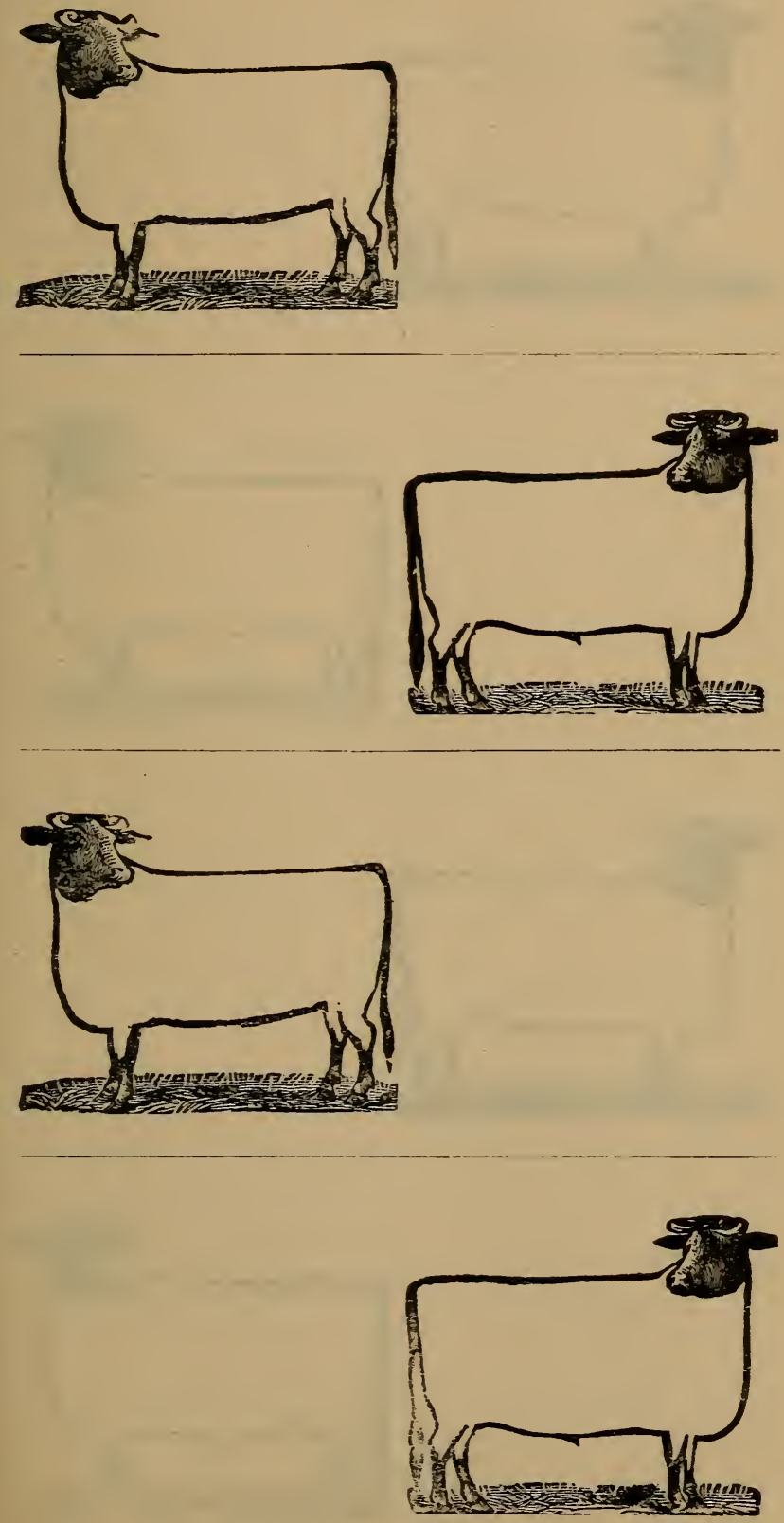


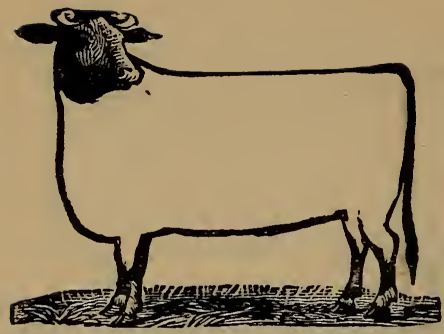

.
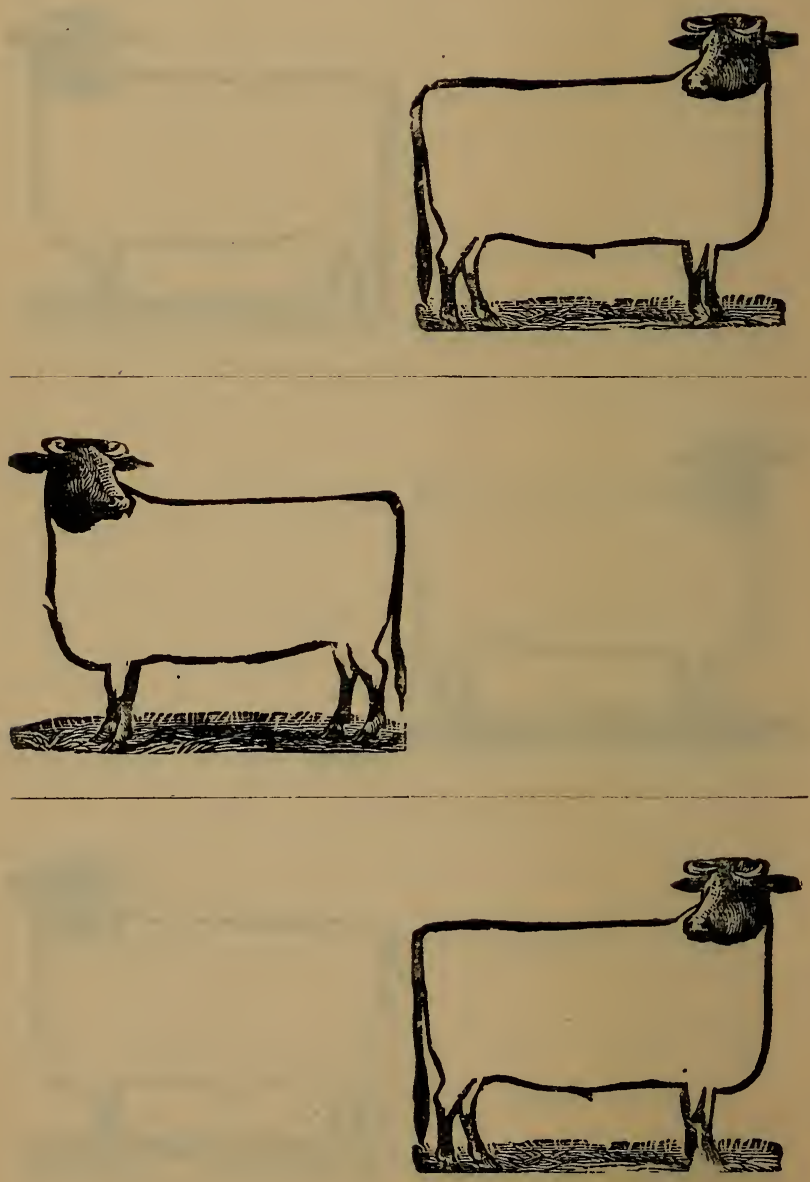

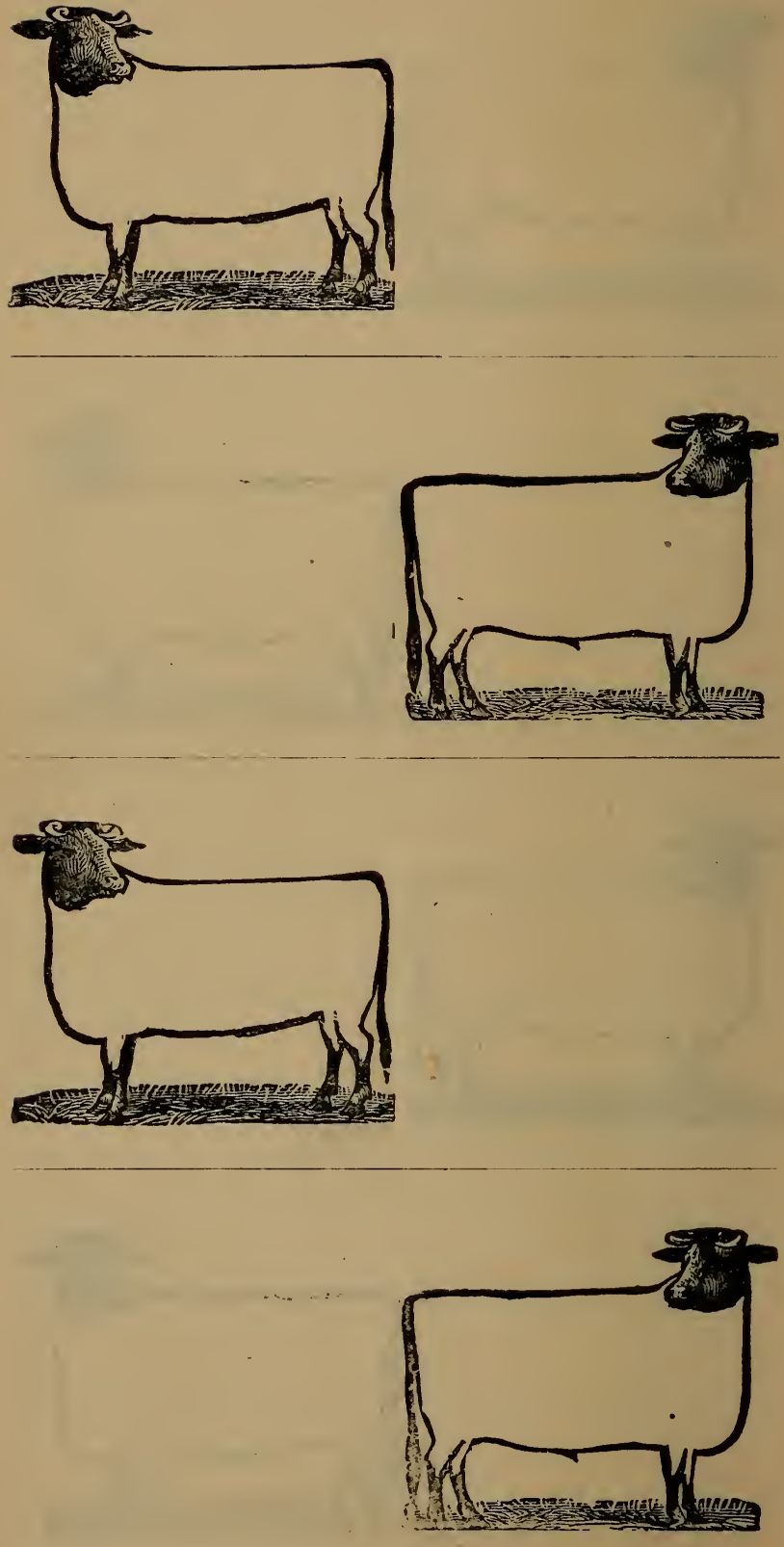



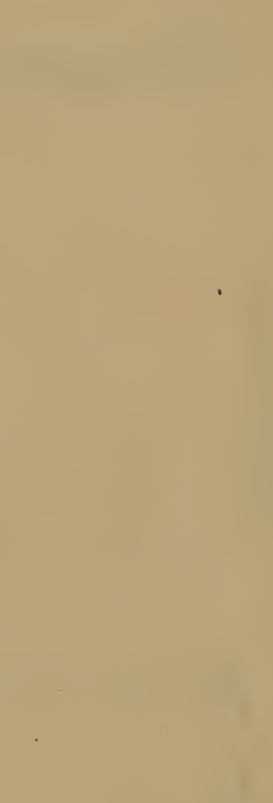


,

- 


\section{GENERAL INDEX.}

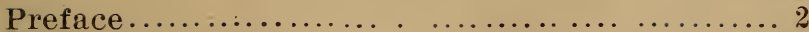

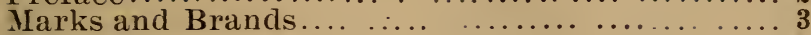

Estray Law. ...........................

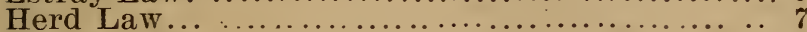

Hide Registry ....................... 8

Criminal Law Relating to Animals.............. 9

Prairie Fires ............................... 10

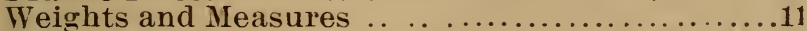

Herd Record...................... 13,14 and 15

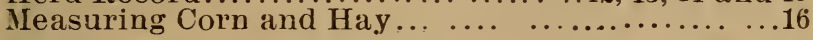

\section{Brand Index.}

All brands having figures are indexed as numerals. All brands containing a sign and letter are indexed as sign brands. All letters containing a bar are indexed as bar brands, Each precinct is indexed separately, both as to brands and names.

\section{Burton Precinct Brands.}

Numerals-17, 19, 20, 22, 23, 25, 26, 27, 29, 30, 35.

Single Letters-17, 21, 29, 33, 34.

Double Letters, separate-18, 20, 24, 26, 27, 30, 31, 32. 33,34 .

Double-Letters, connected-18, 19, 20, 22, 23, 25, 28, $29,30,31,35$.

Sign Brands-17, 18, 19, 22, 23, 24, 25, 27, 28, 29, 30, 31, $32,34,35,36$.

Triple Letters, connected or separate-18, 35 .

Bar, with letters or signs--18, 21, 22, 23, 24. 26, 27, 28, $29,30,32,33$.

Bammerlin, A., Springview................

Bickner, E. P., Springview ................

Brown, W. C., Springview................

Butler, Mrs. C., Mills ......... ...........

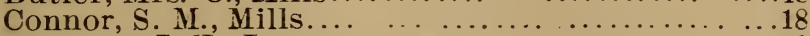

Demmer, J. H., Lutes .........................

Eweing, C. F., Spring view ................. 18

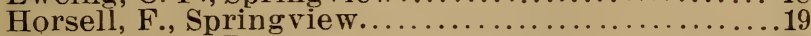

Horsell, Wm., Lutes. . ....... ........... . . .

Hills, C. H., Lutes ........................19

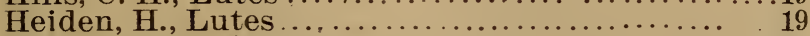

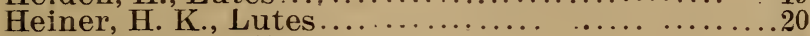

Hamblet. F. H., Lutes ...................20

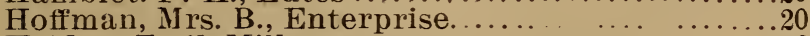

Heiden, Emil, Mills.......................20

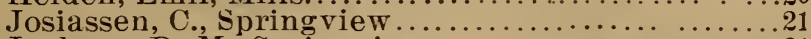

Jackson, D. M., Springview......... . . . ...21

Jackson, J. A., Springview ..................

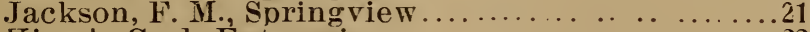

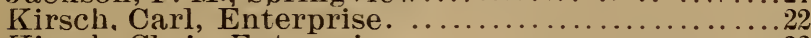

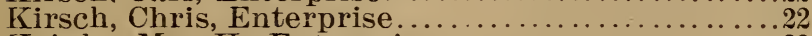

Keinke, Mrs. H., Enterprise ................

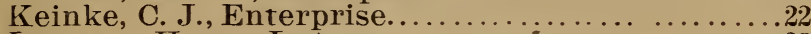

Leavens, Harry, Lutes...... . . . . . . . . . . . 23

Lees, F. J., Enterprise........................

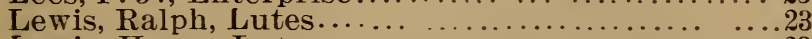

Lewis, Harry, Lutes..................... 23

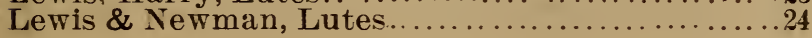


Munger, Chas.. Springriew ..................24

Munger, s. R., Springview....................24

Mays Bros.. Enterprise....................... . . 25

Myers, J. H., Enterprise......................25

Mickey Bros, Springview......................25

Mutz, Otto, Springview .......................... 25

McKee, Herbert, Lutes..............................26

$O^{\prime}$ Neil, J. B., Spring view .........................26

Millikin, Perry \& Co., Springriew. . .. . .........26

McAllister, Wm., Lutes .......................26

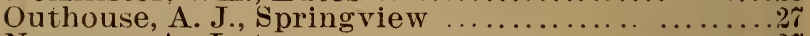

Newman, A.. Lutes................................2

Palmer, L. W. \& G. W., Springview ................2

Page, W G.. Springview .......................2

Rhodes, F. J., Lutes.................................. 28

Robinson, A. E., Lutes...........................28

Shaw, A., Lutes...............................28

Snider, Cyrus, Lutes .................................. 28

Snider, Lee, Lutes................................... 29

Sanderson, G. E., Lutes ... ... .................29

Svlvester, W. B. \& A , Lutes... .. ..............29

Shaw, Henry C., Mills.........................29

Stapleton, Chas., Mills ............................. 30

Shaw, Chas. H., Mills............................... 30

Sprague, G. W., Springview ....................30

Sylvester, Chas., Mills......................... 30

Schoattger, Fred, Enterprise .......................

Skirving, James, Ainsworth . . . . . . . . . . . . . 31

smith, T. V., Springview. . . . ................31

Theide, Carl, Enterprise.........................

Theide, Fred, Enterprise.........................

Tiffany, L. E., Enterprise.........................

Tuton, N. B., Miils............................. 32

Verrell, Dix, Enterprise ...........................

Wiley, Ed, Lutes......................................

wiley. Outarbine, Lutes.............................

wiley, Wm., Lutes..................................

wiley, James, Lutes................................... 33

Warner, W. W., Enterprise.......................... 34

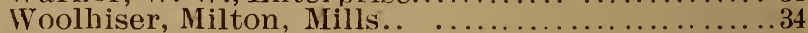

Webster, Chas., Enter prise....................... 34

Webster, C. O., Enterprise.......................

Woolhiser, A., Mills ................................. .

Wood, F. B., Enterprise............................. 35

Wood, M. M.. Enterprise ......................... 35

Wilcutts, F. M., Springview .......................... 35

Wood, John P., Lutes............................... 36

\section{euster Precinct Brands.}

Numerals--37, 40.

Single Letters--42, 43.

Double Letters, separate-37, 38, 39, 40, 41, 4.2.

Double Letters, connected--39, 42, 43 .

Sign Brands--40.

Triple Letters, connected or separate-39, 41 .

Bar, with letters or signs-3i, 38,41 .

Allen, S. S., Springview ......................

Brant Bros., Springview........................

Ballard, George, Springview.........................

Carr, L. R., Springview............................

Carnahan, J. S., Springview ....................... 
Cederberg, Peter, Springview................... 38

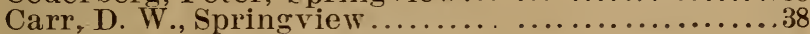

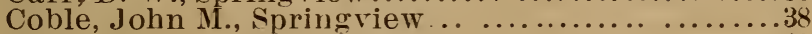

Carr, John F., Springriew $\quad \ldots \ldots \ldots \ldots \ldots \ldots \ldots . \ldots . \ldots$

Fox, Grant, Springriew...................... 39

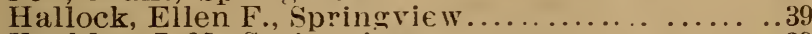

Hackler, J. M, Springriew........................... 39

Johnson, John, Springview ................40

Jaques, T. V., Spring view. . ................. . 40

Monroe, E., Springview....................... 40

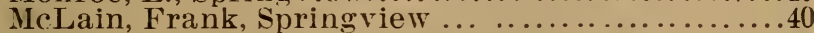

Painter, B. A., Springriew ................41

Skinner, F. W., Springview $\ldots \ldots \ldots \ldots \ldots \ldots \ldots . .41$

Skinner, Thomas, Spring view ................

Skinner, Albert, Springriew .................

Van Houton, C. J., Springview................ 42

Van Winkle, John, Springview................. 42

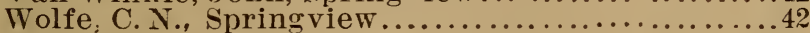

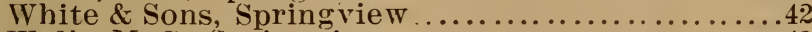

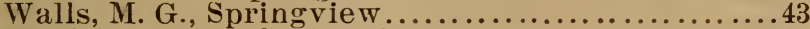

Williams, E. H., Springview............... 43

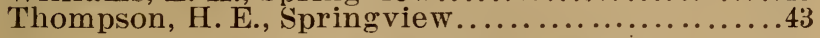

\section{Garfield Precinct Brands.}

Numerals--44, 48 .

Single Letters--47 48.

Double Letters, separate-45, 46, 47, 48, 49 .

Double Letters, connected $-45,46,49$.

Sign Brands.

Triple Letters, connected or separate -43 .

Sign, with letter or letters-44, 48.

Bar, with letters or signs -44 .

Blake, Wm., Springview....................43

Barton, R. A., Springview...........................

Conway, W. K., Springview................ . 44

Dawson, H. M., Springview .... ............44

Dawson, John I., Norden................... 44

Foster, Josephine, Springview ..................

Fessant, George, Spring view................ 45

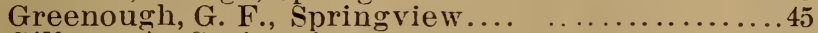

Gilbert, A., Springview.................... 45

Henneman Bros., Springview .. ............46

Herron, F. J., Springview .......................

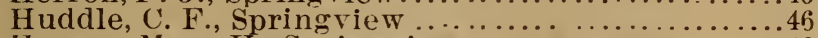

Herron, Mary K., Springview .... . ..........46

Lampitt, Wm., Springview...................

McConnell, C. IV., Springview................

McConnell, Chas., Springview ..................

Overstreet, C. E., Mills.........................

Overstreet, T. J., Springview .....................

Pfaff, D. W., Springview.... $\ldots \ldots \ldots \ldots \ldots \ldots \ldots \ldots \ldots$

Smoot, Robert, Springview........................

Sprague, Wm., Springview......................

Schrantz, J. S., Springview ............... 49

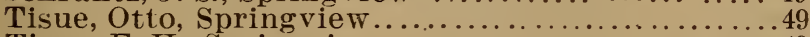

Tisue, E. H., Springview... .................. 49

\section{Holt Precinet Brands.}

Numerals-51, 52, 53, 56, 57, 59, 60 .

Single Letters-50, 51, 59, 60

Double Letters. separate--51, 52, 53, 54. 56, 57. 
Double Letters, connected-- $50,52,56,58,60$.

Sign Brands--55, 36, 59, 61, 54.

Triple Letters, connected or separate-50, 54 .

Bar, with letters or signs $-49,51,52,54$.

Akers, J. W., Springview...................... .49

Austin, W. A., Springview................... 50

Bingham, J. S., Springview ................... 50

Booth, J. G., Springview....................... 50

Ballard, Mary, Springview ...................50

Booth, C. A., Springview............................

Clark, Joseph, Springview....................... . .

Carlson, Alfred, Marlbank ......................

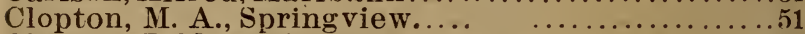

Clopton, I. M., Springview .................... 52

Dixon, H. J., Marlbank........................ 52

Dixon, Frank, Marlbank......................... 52

Eberhart, W. B., Marlbank......................... 52

Fink Bros., Springview.. ........................ 53

Gireau, Paul, Springview..................... 53

Heerton, Eilért, Norden......................53

Hastings, W. J., Springview ........................ 53

Hamilton, L. L., Springview...................54

Heine man, Ed., Marlbank.....................54

Hamilton, C. L., Springview...................54

Jewett, Stephen, Enterprise.................... 54

Kurzenberger, Wm., Springview...................

Kurzenberger, Godfrey, Springview..............

Kuhl, Henry. Springview ......................55

Renaston, Sanford................................

Kenaston, W. A., Springview.......................

Lear, C. E., Springview ........................56

Lear, Wm., Springview .......................56

Loughran, T. F., Springview................. 56

Mock, John, Marlbank .........................

Mock, Mrs. F., Marlbank ...................... 57

Neihus, Claus, Marlbank ....................... 57

Neihus, Frank, Springview .................... 57

Pense, A., Springview ............................. 58

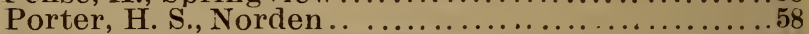

Ruther, Joseph, Springview ....................... 58

Roby, D. K., Spring view ..................... 58

Snyder, E. L., Springview .......................59

Snyder, Henry, Springview ........................

\section{Keya Paha Precinct Brands.}

Numerals--61, 62, 63, 64, 65, 67, 68 .

Single Letters--64, 67 .

Double Letters, separate $-63,66$.

Double Letters, connected--61, 62, 63, 64, 65 .

Sign Brands-62, 64, 65, 68 .

Triple Letters.

Bar, with letters or signs--65, 67, 68.

Adkins, R. M., Brocksburg....................61

Baker, A. J., Brocksburg.......................... 62

Baker, J. H., Brocksburg........................... 62

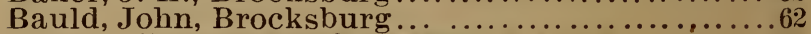

Briscoe, Joseph, Brocksburg........................62

Collins, W. M., Brocksburg .........................63

Doyle, John, Brocksburg.............................. 63

Ditto, Clark, Brocksburg................................6. 63

Hutzel, R. L., Brocksburg .......................6. 
Hudson, W. N., Brocksburg ...............64

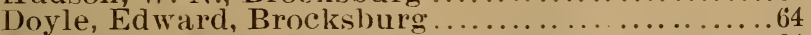

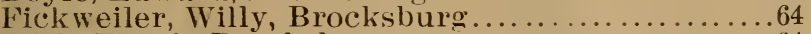

Irish, Frank. Brocksburg.................6. 64

Jeffords, Mary A., Brocksburg..................

Knapp, L. A.. Brocksburg .................... 65

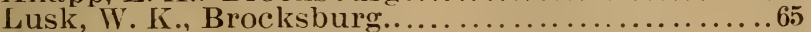

Munt, Frank, Brocksburg.................65

Payn, John, Brocksburg...................66

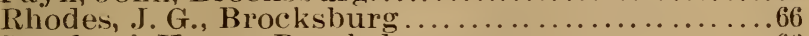

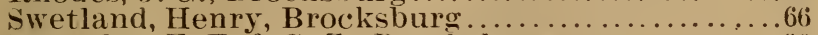

Satterlee, F. E. \& G. Z., Brocksburg...........66

Stewart, Jack, Mills....................6 $6 \tilde{i}$

sullivan, Arthur, Brocksburg.............. $6 \tilde{r}$

Thompson. John, Brocksburg..............6

Vosburg, E., Brocksburg...................67

Wales, H. E., Naper.. ....................68

Wisemiller, Fred, Brocksburg...............6

Williams, M. T., Brocksburg.................68

Wilson \& Olmstead, Brocksburg.............6s

\section{MeGuire Precinct Brands.}

Numerals- $\pi$, $\tilde{\tau}$.

Single Letters-75 and 79.

Double Letters, separate- $\approx 4, \tilde{i} 6$, $\approx s$.

Double Letters, connected- $\pi, \pi 6, \tau, \pi$.

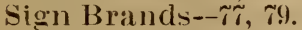

Triple Letters, connected or separate--is.

Bar, with letters or signs-- $\tau t$, $\tau \tilde{\tau}$.

Allen, M. M., Nordell....................

Bassett, W. E., Norden..................

Campbell, J. S., Norden.......................

Evans, T. R., Norden.....................

Englehoff, Fred, Norden ..................

Fisher, F. P., Norden .................

Hutchinson, E. L., Penbrook ................

Hockler, D. M., Norden ........................

Kuhre, Frank, Norden.................... 6

Logan, H. W., Norden.................... 6

Langer, Joseph, Norden......................

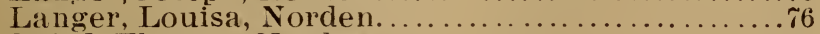

Laird, Thomas, Norden ............... .

McGuire, Mary, M. M Alien, agent, Norden........

Mlorrison, F. N., Norden...................

Poiterin, Aut, Norden ...................

P'orter, J. J., Norden ....................... is

smalley, E., Norden ..................

Thompson, John, Norden..................

Thompson, Jean, Norden..................

Mills, J. B., Norden ......................

Young, Eddie, Norden ....................

\section{Mills Precinct Brands.}

Numerals--69, 70, \%1.

single Letters-- 1 , $\tau$.

Double Letters, separate- $-69,70.73$.

Double Letters, connected-- $70,7 \%, 73$.

Sign Brands--69, $\approx 1, \tau ?$.

Triple Letters, connected or separate.

Bar, witl letters or sigus--69, $70, \tau 1, \tau:$, $\%$.

Anderson, H. D., Mills. 
Billings, Alonzo, Mills . . . . . . . . . . . . . . . 69

Bassett, R. S., Mills......................... 69

- Robinson, S. A., Mills..........................69

Chapin, W. A., Mills.......................

Donason, J., Mills........................

Knight, W. M., Mills...........................

Libolt, Charles, Mills.......................

Pratt, M. E., Mills.........................

Rowan, John M., Brocksburg.....................

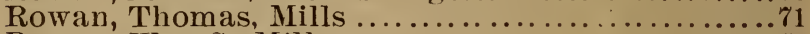

Rowan, Wm. S., Mills........................

Sylvester, Arthur, Mills.....................

Strain, A. F. \& W. S., Mills.................

Stoltenberg, A., Mills......................

Sylvester, Cass, Mills.........................

smothers. Wm., Mills....................

Tuton, Nels, Mills .........................

Wilkinson, George W.., Milis.......................

Wyatt. John, Mills..........................

\section{Norden Precinct Brands.}

Numerals--81, 82, 83.

Single Letters-83, 84 .

Double Letter's, separate--81, 85.

Double Letters, connected--79, 80, 81, 82, 83, 84, 85.

sign Brands--84.

Triple Letters, connected or separate--80.

Bar, with letters or signs--80, 81, 82, 84 .

Brewster, F. L., Norden....................

Billings, $O$. P., Norden .......................

Billings, $\mathrm{O}$. P.Jr., Norden .................. 80

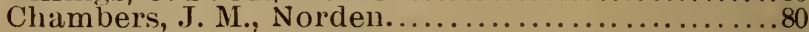

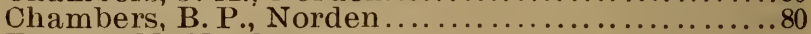

Frauen, M., Norden............................. 80

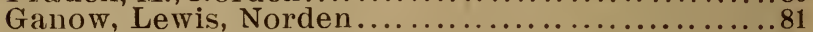

Graddy, B. L., Norden ....................

Kirk, Joseph S., Norden ......................

Kiearville, Wm., Norden ......................

Lawrence, A., Norden...................... 8 .

Luton, A., Norden.......................

Kaplan, J. V., Norden................... 8 .

Luton, J. W.. Norden .........................

Moore, John S., Norden....................... 83

Powell, E. H., Norden ........................83

Payne, Pearl, Sparks....................... 83

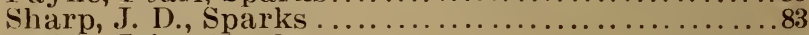

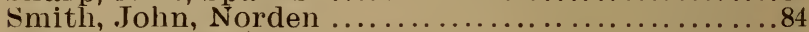

Schifferns, WW. H., Norden................. 84

Siefken, George, Norçen.................... 84

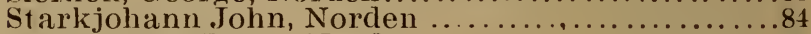

Tschauner, Henry, Norden...................

Zirnig, Frank. Norden ..................... 85

\section{Pine Precinct Brands.}

Numerals--88.

Single Letters--89, 90.

Double Letters, separate-85, 86, 8\%, 88, 89, 90 .

Double Letters, connected-86, 87, 89 .

Sign Brands-88, 90, 8\%.

Triple Letters, connected-or separate-90.

Bar, with letter's or signs--86. 
Amspoker, Ross, Springview ................ 85

Amspoker, s., Springview .................

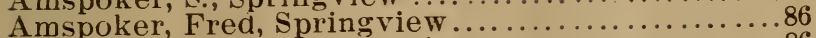

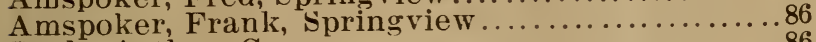

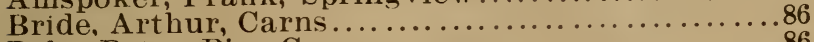

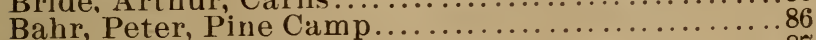

Cowger, John, Springview ............................

Forgey, Wm., Springview . . . . . . . . . . . . . . 87

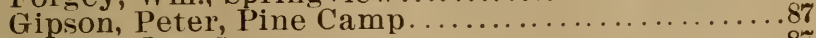

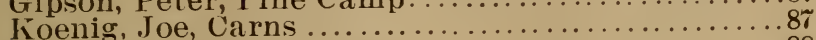

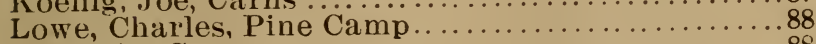

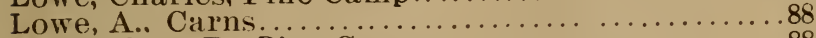

McCrocken, R., Pine Camp.................. . 88

Osborne, W. L., Springview.................. 88

Oamek, Frank, Carns....................... . 89

Prescott, Charles, Carns.................... 89

Paris, E. B. \& Elsie B , Carns... . . . . . . . . . . 89

Paris, R. B.. Carns......................... 89

Peet, M. D., Spring view . . . . . . . . . . . . . . .

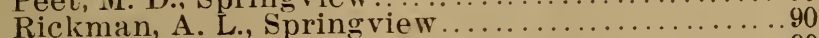

Riley. E., Carns......................... 90

Roe Bros., Carns......................... 90

\section{Simpson Precinct Brands.}

Numerals $-93,94$.

Single Letters-94, 95 .

Double Letters, separate--94.

Double Letters, connected-9.5.

Sign Brands -93, 94, 95.

Triple Letters, connected or separate--93.

Bar, with letters or signs.

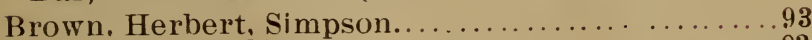

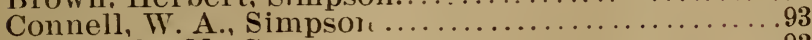

Dyer, John M , Carns....................... 93

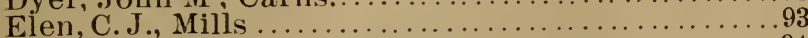

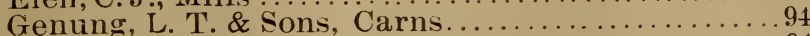

Harvey, $\mathbb{W}$. H. \& Sons, Mills................. 94

Hougen, P., Simpson...................... 94

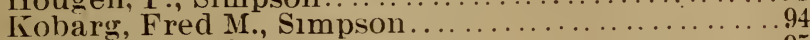

Swaffer, C. J.. Simpson ........................

Thorndyke. A. P., Simpson ................95

Turney, B. S., Simpson ................. 95 
MAY 31902
I COPY DEL. TO CAT. DIY.
MAY 51902
MAY 121902

MAY 31902
I COPY DEL. TO CAT. DIY.
MAY 51902
MAY 121902

MAY 31902
I COPY DEL. TO CAT. DIY.
MAY 51902
MAY 121902

.

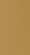 \\ MAY 121902
}

MAY 121902

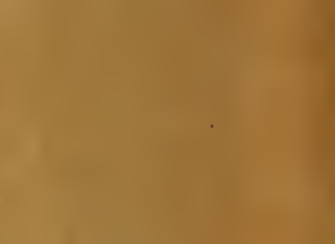




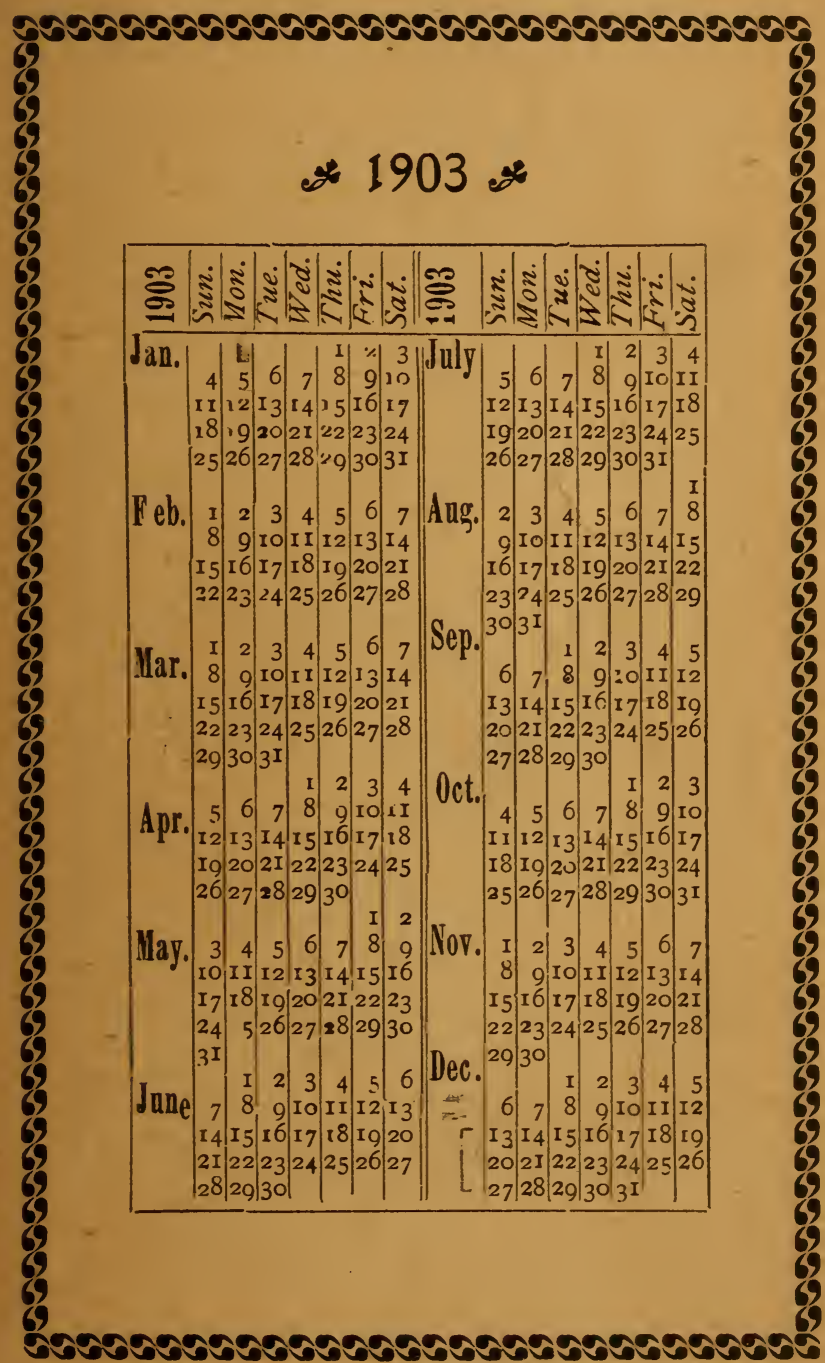


LIBRARY OF CONGRESS

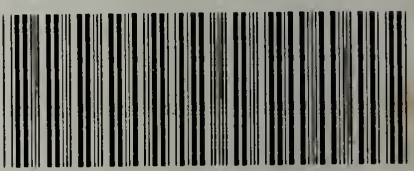

00028307664 